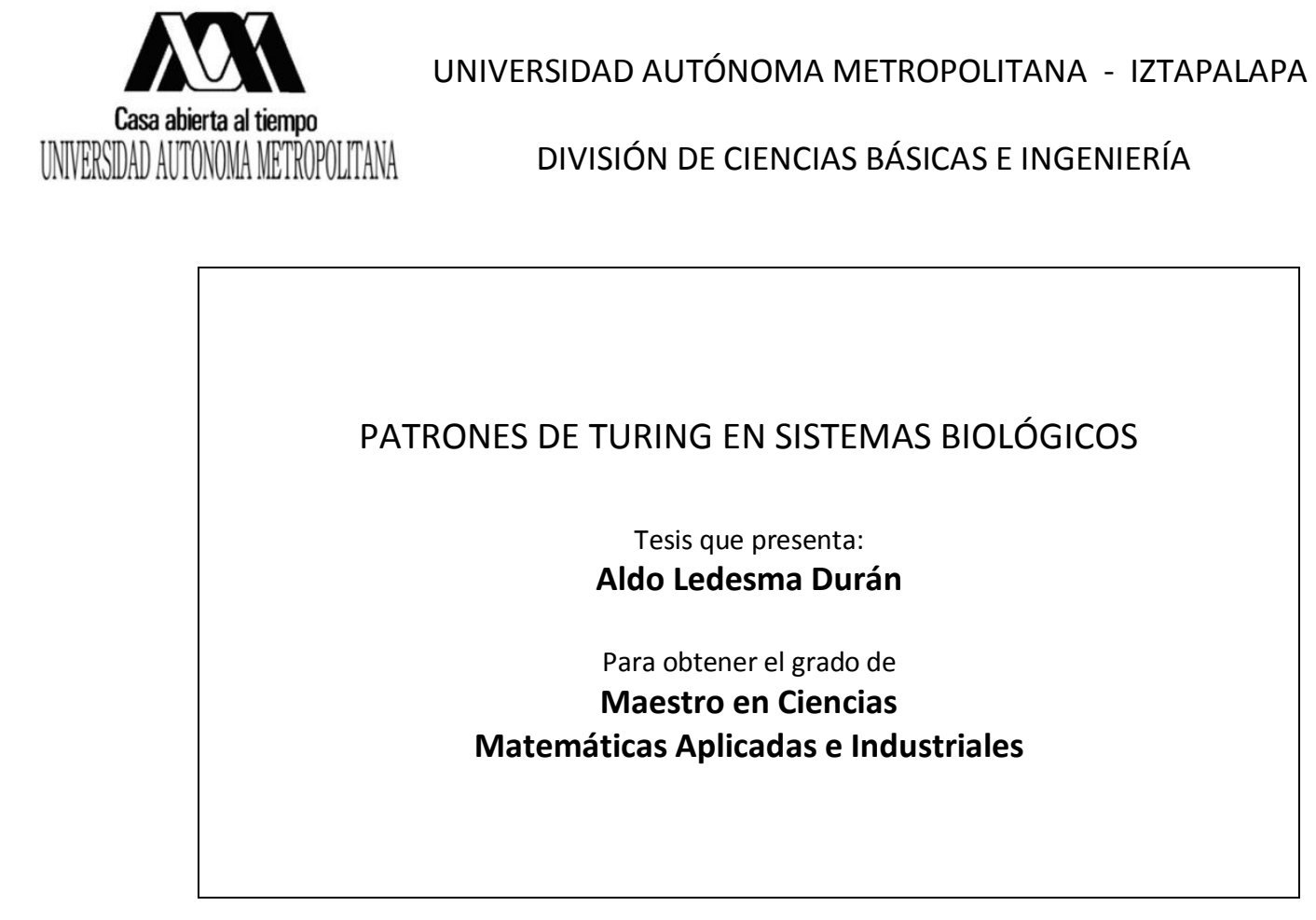

Asesores: Dr. Lorenzo Héctor Juárez Valencia

Dr. Iván Santamaría Holek

Jurado Calificador

Presidente :

Dr. Alfredo Nicolás Carrizosa

UAM-I

Secretario:

Dr. Lorenzo Héctor Juárez Valencia

UAM-I

Vocal:

Dr. Marcos Aurelio Capistrán Ocampo

CIMAT

Vocal:

Dr. Iván Santamaría Holek

UNAM

México, D.F. diciembre 2012 



\section{Resumen de la tesis}

La formación de patrones espacio-temporales es una de las características más importantes de los sistemas biológicos debido a que pueden ser determinantes tanto en el desarrollo y la función del organismo como en su supervivencia y adaptación al medio ambiente. En particular, uno de las manifestaciones de gran interés consiste en la formación de los patrones espaciales en la piel de algunos animales. La teoría más ampliamente aceptada que explica la formación de estas estructuras supone que un patrón químico inicial (genético), que en general puede depender de las condiciones externas, es responsable de que las células se diferencien siguiendo rutas metabólicas específicas que las llevan a formar los patrones que observamos, por ejemplo, en la piel. En el enfoque matemático del problema, estas suposiciones pueden modelarse con bastante precisión en términos de sistemas de ecuaciones diferenciales parciales no-lineales que se conocen como ecuaciones de reacción difusión (RD). Estas ecuaciones acoplan los procesos de transporte de masa y de reacciones químicas que son necesarios para dar una explicación cuantitativa de las observaciones experimentales. Los patrones que reproducen son

conocidos como estructuras de Turing y fueron identificadas en el laboratorio sólo recientemente. En este trabajo se estudian primeramente los conceptos físico-químicos y biológicos involucrados en las ideas de Alan Turing, para luego establecer el sistema de ecuaciones diferenciales parciales que resultan de un mecanismo RD, y finalmente arribar a las condiciones matemáticas para producir patrones espaciales estacionarios. Después, describiremos los métodos de aproximación de diferencias finitas y elemento finito que nos permitirán resolver numéricamente estas ecuaciones, métodos que fueron programados en el lenguaje Matlab para el caso de una y dos dimensiones y distintas condiciones de frontera. Estos programas permitirán reproducir algunos resultados reportados en la literatura. Como contribución original, en este trabajo propondremos algunas modificaciones a los modelos existentes que permitirán comprender mejor los patrones que se encuentran en la piel de los peces Pseudoplatystoma. 


\section{Agradecimientos}

A los doctores Alfredo Nicolás, Héctor Juárez, Iván Santamaría y Marcos Capistrán por el tiempo y la dedicación que empeñaron en la lectura, revisión y corrección de este trabajo. Esta tesis fue realizada con el apoyo de la beca para estudios de

maestría de CONACyT y la beca PAPIIT-UNAM del proyecto ID100112. 


\section{Índice general}

1. Introducción 1

1.1. Panorama histórico del problema . . . . . . . . . . . . 1

1.2. ¿Qué es un patrón de Turing? . . . . . . . . . . . . . . 6

1.3. Objetivos de esta tesis . . . . . . . . . . . . . . 9

2. Preliminares 11

2.1. Sistemas de ecuaciones diferenciales . . . . . . . . . 11

2.2. Reacción oscilante Belouzov-Zhabotinski . . . . . . . . . . 15

2.3. Difusión . . . . . . . . . . . . . . . . . . 20

3. Patrones de Turing 23

3.1. Condiciones matemáticas para la aparición de patrones . . . . 23

3.2. Ejemplo: reacción de Schnakenberg . . . . . . . . . . . . . 30

4. Métodos numéricos 37

4.1. Diferencias Finitas . . . . . . . . . . . . . 38

4.1.1. Ecuaciones en diferencias finitas . . . . . . . . . . 38

4.1.2. Discretización espacial y temporal . . . . . . . . . . . . 39

4.1.3. Condiciones de frontera . . . . . . . . . . . . . . . 40

4.1.4. Esquemas de diferencias finitas para la ecuación RD . . 42

4.2. Elemento Finito . . . . . . . . . . . . . . 46

4.2.1. Discretización temporal . . . . . . . . . . . . 47

4.2.2. Formulación variacional . . . . . . . . . . . . . 48

4.2.3. Funciones base y proyección en elementos . . . . . . . . 49 
5. Miscelánea de patrones obtenidos por simulación numérica 53

6. Patrones en peces Pseudoplatystoma 67

6.1. Antecedentes . . . . . . . . . . . . . 67

6.1.1. Morfología de los surubíes . . . . . . . . . . . 67

6.1.2. Modelos de patrones en peces . . . . . . . . . . 69

6.1.3. El modelo BVAM . . . . . . . . . . . . . . . 70

6.2. Simulaciones numéricas . . . . . . . . . . . . . . 72

6.2.1. Condiciones iniciales y de frontera . . . . . . . . . . 73

6.2.2. Coeficientes dependientes del espacio . . . . . . . . 76

6.2.3. Sistema reacción-convección . . . . . . . . . . . . 80

6.3. Discusión . . . . . . . . . . . . . . . . . . 81

$\begin{array}{lr}\text { Conclusiones y Perspectivas } & 85\end{array}$

$\begin{array}{lr}\text { Bibliografía } & 89\end{array}$

$\begin{array}{ll}\text { Apéndice A } & 95\end{array}$

$\begin{array}{ll}\text { Apéndice B } & 101\end{array}$

$\begin{array}{lr}\text { Apéndice C } & 103\end{array}$

$\begin{array}{ll}\text { Apéndice D } & 107\end{array}$ 


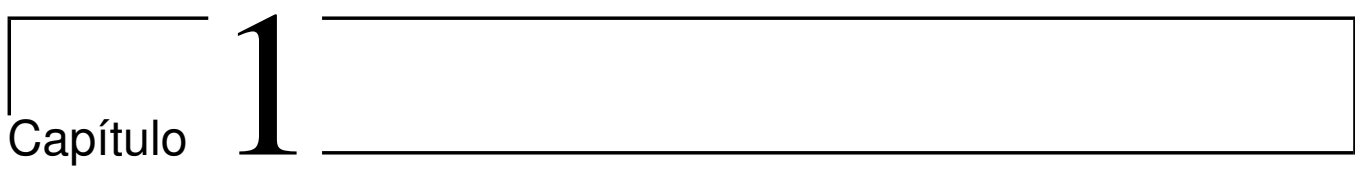

\section{Introducción}

En este capítulo se explica sucintamente qué es un patrón de Turing enmarcando el problema en un contexto histórico, además de prefigurar los conceptos necesarios para su estudio que se detallarán en el siguiente capítulo. Se destaca la importancia de las ideas de Turing en el campo de las biomatemáticas, dejando así implícita la motivación de esta tesis. Esta introducción sirve también de glosario de algunos conceptos de física, química y biología con los que el lector pudiera no estar familiarizado.

\subsection{Panorama histórico del problema}

Durante cientos de años, fue (o quizá sigue siendo) un prejuicio el hecho de que las formas más complejas de la naturaleza sólo pueden obtenerse por el planeado y meticuloso diseño de un arquitecto divino, ya sea llamado dios, casualidad o naturaleza. Hasta mediados del siglo pasado era increíble pensar que la formación de las dunas de arena, el complicado diseño de la piel de un leopardo o el inteligente diseño de una colmena pudieran explicarse meramente con las frías leyes de la química o la física. Del mismo modo, todo aquello que representa la vida, como sus formas, su diseño, sus colores o su funcionamiento, estaban más allá de alcance de una ley, no solo por su complejidad inherente e incuestionable, sino porque (implícita o explícitamente) se creía que su esencia estaba un escalón más allá de lo que un científico podría capturar en sus modelos [1].

Fue a partir del pensamiento del científico escocés D’Arcy Thomson (1860- 


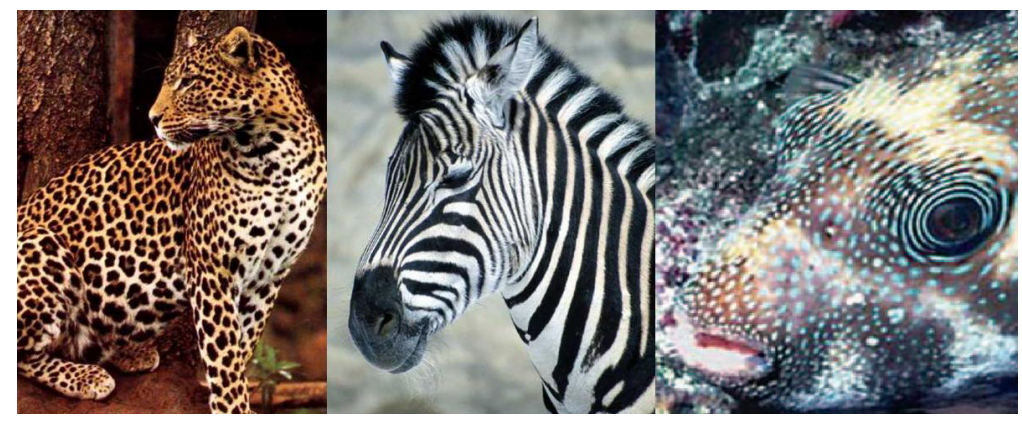

Figura 1.1: Patrones en la piel de algunos animales.

1948) que se pudo iniciar un cambio de mentalidad respecto a la aparición de patrones en los animales. Para él, la selección natural planteada por Darwin (y aceptada hasta nuestros días) tiene el defecto de que no describe el proceso o mecanismo subyacente. Por ejemplo, para explicar la piel manchada de una jaguar (Ver Figura 1.1), la explicación evolutiva es que la selección natural prefirió este tipo de superficie para que el felino pudiera camuflarse entre la espesa vegetación y acechar más fácilmente a su víctima. Esta explicación, aunque parcialmente cierta, adolece de un defecto: no establece cómo la selección natural conforma ese patrón, o de qué armario de pieles elige la naturaleza el más adecuado para el jaguar, o cómo se adapta a los cambios evolutivos que ha sufrido esta especie a lo largo del tiempo [2]. Estas dudas razonables de Thompson fueron consideradas prácticamente una herejía por los biólogos de sus tiempos.

Las primeras pistas de cómo un cúmulo de células pueden diferenciarse y formar un patrón en la piel de los animales provienen no de la Biología, sino de la Física donde ya se conocían algunas propiedades de los sistemas complejos. En particular se sabía que el orden en un sistema de muchas partículas no podía ocurrir espontáneamente, debido a la segunda ley de la Termodínamica, sino que requería que que el sistema estuviera abierto y utilizara energía. Ilya Prigogine definió a los sistemas auto-organizantes de este tipo como estructuras disipativas, de las cuales un ser vivo es un claro ejemplo [3]. Otro indicio de la Física es el hecho de que el orden y simetría de un sistema usualmente van en sentidos contrarios, esto es, que conforme más simetría tiene un sistema, más desordenados están sus unidades o componentes y viceversa. Como ejemplo de lo primero, tómese las moléculas de un fluido que viajan completamente erráticas y sin embargo el fluido es 
igual por donde se le vea. Mientras tanto en el caso opuesto, las moléculas de un cristal se encuentra perfectamente organizadas en los vértices del poliedros y, no obstante, sólo tienen limitados ejes de simetría. La existencia de una estructura organizada requiere de una fuerza termodinámica que produzca el llamado rompimiento de la simetría[4].

Los seres vivos somos sistemas complejos, es decir sistemas termodinámicos formados por muchas celdas llamadas células. Evidentemente somos sistemas abiertos que intercambian materia y energía con su entorno, mismas que le ayudan a gestarse, desarrollarse y reproducirse. El comer, respirar, asolearse, beber, representan la fuente que nos proveen de lo necesario para vivir. Todos estos intercambios son reacciones químicas, lo cual nos hace pensar en éstas como la fuerza termodinámica que permite a las células diferenciarse en órganos bien identificados y en patrones, es decir, generar un orden [5].

En la década de los años 50's, Belousov descubrió que en una reacción química con dos distintos tipos de iones de Cerio, la mezcla no alcanzaba un estado estacionario después de un proceso más o menos uniforme (como se observaba de costumbre), sino que oscilaba entre la preponderancia de una y otra especie química. Esto se notaba en la alternancia sucesiva de dos coloraciones características de las distintas especies. Esta fue la primera vez que se observó una reacción química oscilante, y con ello el ordenamiento aparentemente espontáneo de un sistema. En un inicio hubo un rechazo de la comunidad científica de la época a estas observaciones, puesto que se creía que violaban la segunda ley de la termodinámica. Según su interpretación más conocida, esta ley física establece que el curso de la naturaleza avanza sólo en cierta dirección dictada por el aumento de la entropía y, por ende, del desorden. Esto quiere decir que la manzana cae del árbol al suelo, el metal limpio se oxida y la gota de crema se difunde en el café y no a la inversa. El punto de los detractores era que un sistema no puede avanzar espontáneamente a un sistema más ordenado, es decir, la crema en el café no se concentrará por sí misma para rehacer la gota inicial. Era como si Belousov propusiera que la crema por sí misma se difunde y concentra una y otra vez en el café de manera natural, lo cual parecía un sinsentido. Por lo tanto, sus resultados fueron ignorados por más de 20 años, hasta que su compatriota Anatol Zhabotinsky realizó un meticuloso experimento que completó y confirmó las observaciones de Belousov, es decir, que hay sistemas químicos que pueden oscilar y crear un orden temporal. A partir de estas aportaciones, la reacción se conoce como la reacción $\mathbf{B Z}$, por los nombres de sus 


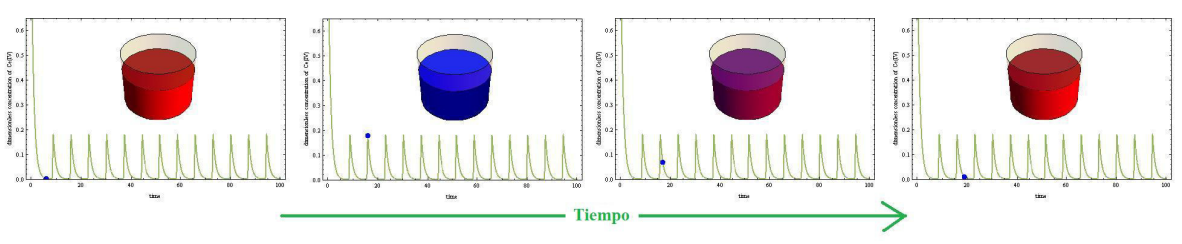

Figura 1.2: Oscilaciones temporales en la reacción BZ

descubridores [6]. La figura 1.2 ilustra este fenómeno.

La aparente contradicción con la segunda ley se resuelve notando que ésta dice que en un sistema cerrado la entropía no decrece entre dos estados de equilibrio, pero no dice nada sobre lo que pasa en el intermedio de la reacción. En el caso de la reacción BZ, el orden generado por las oscilaciones sucesivas no dura eternamente, sino que después de ciertas repeticiones el sistema deja de reaccionar. Al alcanzar el estado estacionario, la entropía del sistema (considerando la mezcla y sus alrededores) efectivamente es mayor que la que tenía antes de empezar la reacción, en perfecta concordancia con lo establecido con la segunda ley, sólo que en el camino hacia el estado estacionario, la mezcla transita una ruta sinuosa y extraña para aquello que los investigadores conocían en esos tiempos [7]. Hoy en día esa extrañeza ha dejado de serlo. Hoy se sabe que los sistemas en equilibrio descritos por la termodinámica clásica describen muy pocas situaciones relevantes para la biología. El planeta, el clima, la vida misma, están descritos por procesos fuera del equilibrio, con intercambios que se activan continuamente a tal grado que para aspirar a estar en equilibrio termodinámico, esencialmente hay que primero estar muerto. Este devenir de la vida necesita para su evolución de energía continua.

La clave en la reacción BZ es la auto-catálisis, en la cual un compuesto acelera la taza con que ocurre la producción de sí mismo. A este compuesto se le llama catalizador al que, en el caso de reacciones bioquímicas, se le conoce también como enzima. Lo particular de las reacciones del tipo BZ es que hay dos catalizadores X y Y que, aparte de producirse a sí mismos, inhiben al otro, dando lugar a un proceso competitivo entra ambos que genera la preponderancia alternada de uno (que pinta la mezcla de un color, digamos rojo) y de otro (que la pinta de azul), lo cual produce las oscilaciones y los cambios de color de la mezcla[8].

Sin embargo, prácticamente el mismo mecanismo de oscilaciones temporales puede dar lugar a la creación de patrones en el espacio; la diferencia 


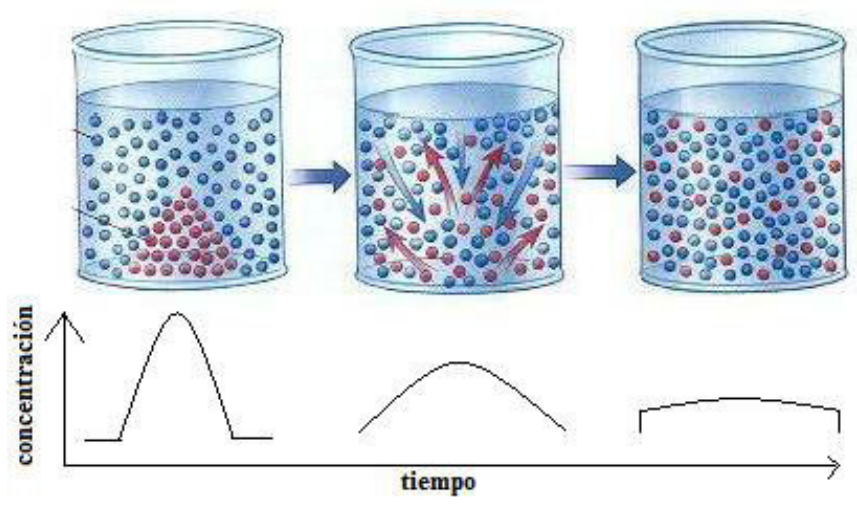

Figura 1.3: La difusión como proceso estabilizador resulta del movimiento térmico de las partículas del medio.

consiste en la manera como se lleva a cabo la reacción. En el experimento de Belouzov (al igual que en las mezclas químicas estándar) todos los compuestos se introducen en un vaso de precipitado con un agitador magnético que mezcla continuamente todos los componentes para que la concentración de cada uno de ellos sea homogénea en todo el sistema . Sin embargo, si los mismos compuestos se introducen en un sistema donde no hay mezclado, es decir, donde la única fuente de movilidad de los catalizadores es la difusión molecular, entonces las pequeñas variaciones locales de concentración de los compuestos X y Y pueden dar lugar a que el estado de la reacción sea distinto de un lugar en otro, y que mientras en algún sitio prepondere el rojo, en otro sea azul. El punto clave de estos patrones transitorios es que, en ausencia de mezclado, la difusión lleva a cabo el transporte entre las moléculas de los compuestos. La difusión es un proceso espontáneo por el cual las moléculas de los reactantes se esparcen en todas direcciones a causa de los choques con las moléculas del medio o solvente como se muestra en la Figura 1.3. Cuando no hay mezclado, la difusión controla la cantidad disponible de un reactante en una región, dando lugar a las variaciones locales y, por ende, a la aparición de patrones [9].

Lo anterior se ha obtenido experimentalmente en medios como el gel. Lo que se ha observado es que estas variaciones no se propagan de manera completamente aleatoria, sino como anillos concéntricos o espirales que irradian hacia afuera, como cuando se tira una piedra en el agua [10]. Estos patrones espaciales se forman por un tiempo limitados hasta que la mezcla 
deja de reaccionar y desaparecen. La etapa transitoria se deriva del hecho de que, por sí solas, tanto las reacciones químicas como la difusión son procesos homogeinizadores que tenderán a borrar cualquier diferencia local, es decir, cualquier patrón.

\section{2. ¿Qué es un patrón de Turing?}

La genialidad del matemático inglés Alan Turing radica en notar que, si bien ambos procesos (la reacción química y la difusión molecular) son homogeneizadores por separado (por lo cual cualquier irregularidad está condenada a desaparecer), juntos pueden dar lugar a patrones estacionarios cuando se les combina de una forma especial. En su famoso artículo de 1952: The chemical basis of morfogenesis [11] Turing establece: primero, cómo se pueden formar patrones químicos espaciales, y segundo, cómo relacionar estos patrones con aquellos observados en la naturaleza.

La morfogénesis (del griego morphê, forma, y génesis, creación) es el proceso por el cual se van desarrollando en un embrión los órganos diferenciados de un adulto a partir de estructuras indiferenciadas. Conjuntamente con el control del crecimiento celular y de la diferenciación celular, constituye uno de los aspectos fundamentales de la biología del desarrollo. Turing propuso que la generación de forma y orden en un ser vivo puede relacionarse directamente con el orden generado con un pre-patrón químico de la siguiente manera: supongamos que en el medio celular del cigoto se puede generar un patrón químico mediante algún mecanismo; supongamos que en las células existen sustancias capaces de responder a la concentración local de ese químicos (los llamados morfógenos), de tal forma que si esa concentración sobrepasa cierto valor, la célula expresa un rasgo (como el color rojo), y si no sobrepasa ese umbral, entonces expresa otro (se torna color azul). El resultado final es la traducción de un pre-patrón químico en una diferenciación de órganos, de tejidos, o patrón en la piel, etc.. Por ello, lo único que hace falta es establecer las condiciones en las que se puede establecer el pre-patrón químico mediante algún mecanismo. Ver Figura 1.4. El mecanismo que él encontró fue del tipo reacción-difusión ( $\mathrm{RD}$ en adelante).

Puesto en otras palabras, el esquema de Turing es el siguiente: un compuesto X lleva a cabo una reacción autocatalítica para generar más de ella misma. La velocidad con la que $\mathrm{X}$ se genera depende de la cantidad de $\mathrm{X}$ preexistente. Pero en este esquema X también activa la formación de un com- 

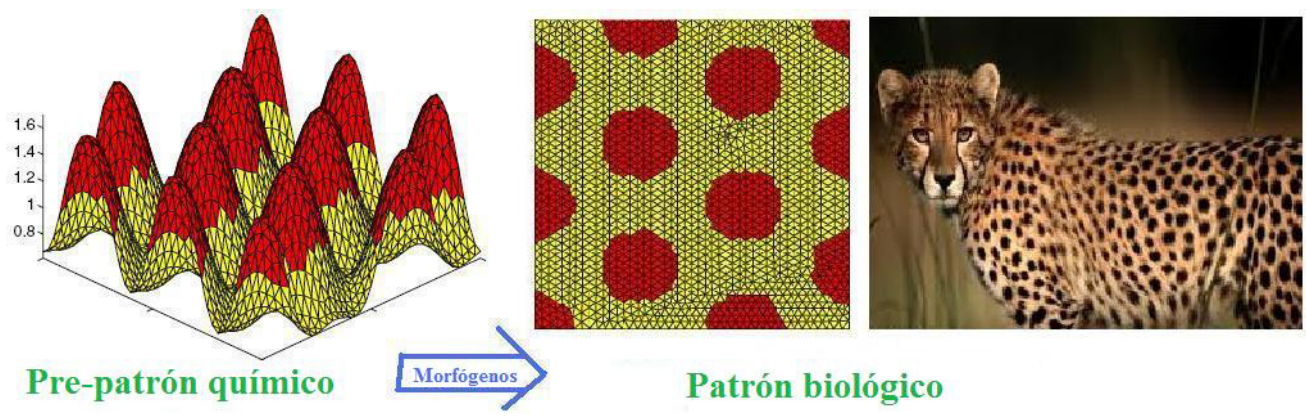

Patrón biológico

Figura 1.4: Según Turing un patrón químico puede generar un patrón biológico mediante los llamados morfógenos que ayudan a la célula a diferenciarse de un color o de otro.

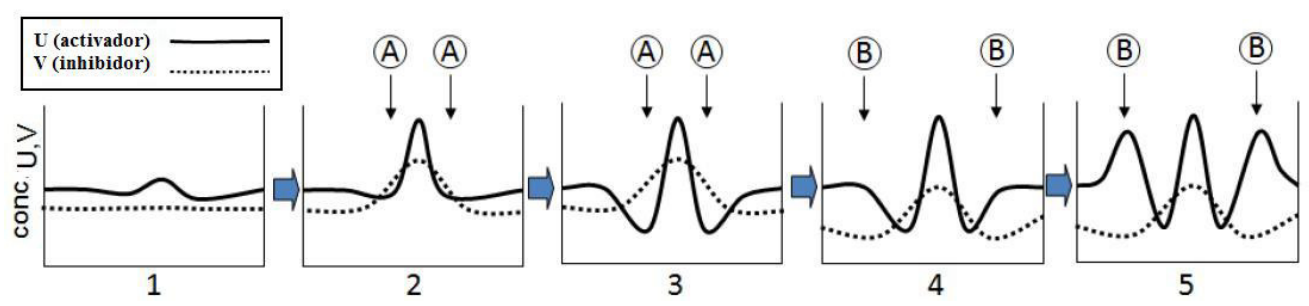

Figura 1.5: Mecanismo RD de Turing. Si en el proceso competitivo entre activador e inhibidor el primero se difunde más lento, se da lugar a irregularidades locales que forman un patrón.

puesto Y que inhibe la formación de más X. Si ambas sustancias se difunden con distintas velocidades en el medio, los rangos de sus respectivas influencias pueden variar localmente, es decir, X y Y pueden dominar en distintas regiones. El punto clave es precisamente la difusión diferenciada que permite que la competencia entre ambos compuestos no sea idéntica en toda la región [13]. Ver Figura 1.5.

En el lenguaje de los químicos, el mecanismo de Turing representa la competencia entre la activación de un compuesto X y la inhibición de un compuesto Y. Para que este esquema pueda dar lugar al desarrollo de patrones, el inhibidor debe difundirse más rápidamente que el activador, porque de otro modo, el inhibidor agotaría por completo la presencia de X en la región y se terminaría la reacción. Además, el mecanismo de Turing es espontáneo y global, a diferencia del esquema BZ donde debe haber perturbaciones locales en el medio para que aparezcan diferencias de concentración [24]. 

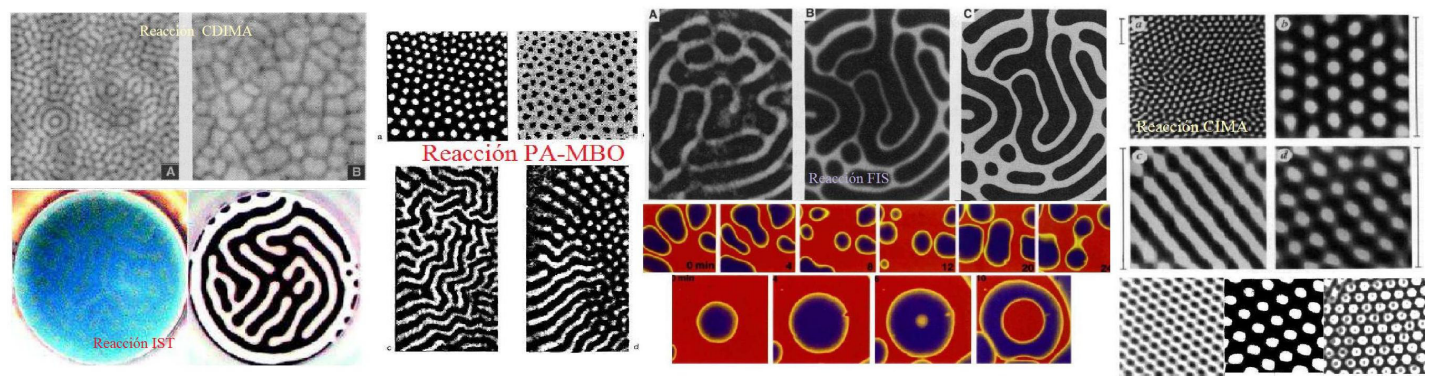

Figura 1.6: Evidencia experimental de patrones de Turing para varias reacciones químicas. Imágenes tomadas de [14, 15, 16, 17, 18, 19, 20, 21, 22, 23].

Turing demostró la existencia de patrones matemáticamente, aunque ciertamente bajo suposiciones muy restrictivas. Por ejemplo supuso un dominio unidimensional, una reacción química muy sencilla y un análisis de las ecuaciones diferenciales sólo hasta orden lineal. Por ello, al igual que pasó a Belousov, la consideración e interés en la propuesta de Turing tuvo que esperar casi 20 años, hasta que la química le prestara atención a los proceso de no equilibrio, y la física-matemática ganará experiencia en el estudio de los problemas no lineales. De hecho, una gran cantidad de evidencia experimental se ha recabado demostrando que un mecanismo puede dar lugar a patrones químicos reproducibles en los laboratorios; ver Figura 1.6 para referencias. Desde entonces el mecanismo de Turing ha gozado de gran popularidad entre los biomatemáticos como explicación de los patrones en la naturaleza, aunque ciertamente no sucede así entre la comunidad de biológos que aún miran la sencillez y elegancia del modelo de Turing con ojos de desconfianza. Los principales argumentos en contra del modelo de Turing son la falta de información biológica incorporada al modelo, la complejidad del proceso de diferenciación celular sobre-simplificado por Turing y, mayormente, la falta de alguna evidencia experimental biológica concluyente [10].

Esto último no ha impedido que desde hace unas décadas para acá se hayan escrito y publicado una gran cantidad de artículos y documentos alrededor del tema, ya sea reformulando las ideas de Turing, reproduciendo patrones por computadora con extraordinario parecido a los encontrados en los animales, o incluso usando las mismas ecuaciones para otras aplicaciones en los campos de la fisiología, la ecología y hasta en el procesamiento de imágenes[25]. Todavía hoy, gente como Murray, Meinhardt, Barrio, Madzva- 
muse, Gierer, Maini, Kondo, etc. ${ }^{1}$ siguen ampliando nuestro conocimiento e interés en las ideas de Alan Turing.

\subsection{Objetivos de esta tesis}

Dada la importancia de las ideas de Alán Turing en el campo de las Biomatemáticas, en esta Tesis nos proponemos revisar su trabajo con los siguientes objetivos concretos:

1. En la Introducción y en el Capítulo 2 ("Preliminares") presentaremos un resumen de los conceptos físico-químicos y biológicos fundamentales que intervienen en la idea de Turing para la formación de patrones: reacciones químicas oscilantes, difusión molecular, ecuaciones que rigen estos dos procesos, morfógenos, etcétera. En primer lugar discutiremos el origen y características dinámicas y de estabilidad de las ecuaciones de la cinética química para reacciones catalíticas oscilantes. En segundo lugar presentaremos un resumen de la teoría de transporte para procesos de difusión. Estos aspectos se resumirán con vista a la dinámica espacio temporal de morfógenos.

2. En el Capítulo 3 ("Patrones de Turing") nos enfocaremos al estudio de los patrones de Turing al analizar las condiciones matemáticas sobre las ecuaciones RD que permiten la existencia de patrones espaciales estacionarios.

3. El capítulo 4 ("Métodos numéricos") está dedicado al análisis de los métodos numéricos que nos servirán para encontrar las soluciones aproximadas de las ecuaciones RD. Esto incluye distintas variaciones de Diferencias Finitas y el método de Elemento Finito.

4. La elaboración de los códigos y su validación al reproducir patrones reportados en la literatura se hará en el Capítulo 5 ("Miscelánea de patrones obtenidos por simulación numérica"). Esto nos permitirá tanto estudiar las características de estos patrones como verificar la corrección de nuestros programas.

\footnotetext{
${ }^{1}$ En la bibliografía y en el texto aparecen las referencias de algunos de los trabajos más importantes de estos autores.
} 
5. Finalmente, en el Capítulo 6 ("Patrones en peces Pseudoplatystoma") presentaremos un estudio numérico original realizado para reproducir algunos de los patrones de una variedad de peces neotropicales. 


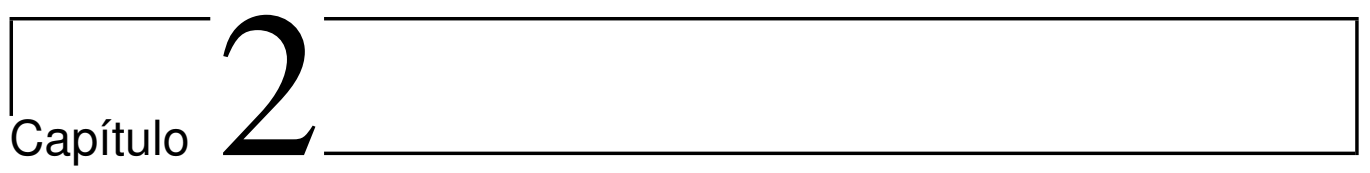

\section{Preliminares}

Como se ha visto, un patrón de Turing resulta de la combinación de reacciones químicas oscilantes y la difusión diferenciada de ambos catalizadores. En este capítulo se adentra al lector en el desarrollo de estos temas, primero considerando la reacción oscilante más famosa, i.e. la reacción BZ, para luego estudiar algunas propiedades básicas de la difusión, de tal manera que podamos arribar a las ecuaciones RD. Sin embargo, para entender cómo aparecen y se desarrollan las oscilaciones temporales y patrones espaciales, así como demostrar su existencia a partir del modelo, es necesario un análisis de estabilidad del sistema, por lo que antes de entrar en materia, damos un ligero repaso de estos métodos tradicionales de análisis de EDO's.

\subsection{Sistemas de ecuaciones diferenciales}

\section{Trayectorias y Equilibrio ${ }^{1}$}

Suponga que el estado de un sistema está descrito por el par de funciones $u(t)$ y $v(t)$. Estas variables de estado dependen de una variable dependiente $t$ que usualmente es el tiempo, y rigen al sistema por el par de ecuaciones diferenciales:

\footnotetext{
${ }^{1}$ La mayor parte de esta sección se extrajo de las referencias [26, 27].
} 


$$
\begin{gathered}
\dot{u}=f(u, v), \\
\dot{v}=g(u, v),
\end{gathered}
$$

en donde el punto superior indica derivación respecto al tiempo. En notación vectorial, este sistema puede escribirse como $\dot{\mathbf{w}}=\mathbf{R}(\mathbf{w})$ y es llamado autónomo porque la función $\mathbf{R}$ no depende explícitamente del tiempo. Las soluciones $u(t)$ y $v(t)$ de un sistema bidimensional forman un conjunto de trayectorias (líneas de flujo u órbitas) que cubren densamente el plano $(u, v)$, ya sea total o parcialmente, suponiendo que los datos son suficientemente regulares. A una figura que delinea ciertas trayectorias elegidas se le llama diagrama fase y al plano $(u, v)$ se le conoce como plano fase. Se puede elegir una trayectoria específica requiriendo que pase por un cierto punto $\left(u_{0}, v_{0}\right)$ de valores iniciales:

$$
u\left(t_{0}\right)=u_{0}, \quad v\left(t_{0}\right)=v_{0}
$$

a un tiempo prescrito $t_{0}$ que en un sistema autónomo se elige usualmente como $t_{0}=0$.

Si uno se imagina a una partícula flotando sobre el plano $(u, v)$, la tangente de su trayectoria está determinada por las funciones $f$ y $g$, que son iguales a $\dot{u}$ y $\dot{v}$, las velocidades horizontal y vertical, respectivamente. El conjunto de todas las trayectorias que empiezan de alguna región del plano w es llamado el flujo de dicha parte.

En el análisis de sistemas de EDO's (Ecuaciones diferenciales ordinarias) son de especial interés los puntos de equilibrio que están definidos como aquellos que cumplen:

$$
\dot{u}=0 \quad \dot{v}=0 .
$$

En este conjunto, también llamado de puntos singulares, críticos o fijos, el sistema está en reposo, es decir, las soluciones que en algún instante crucen este punto permanecerán ahí para todo instante futuro, y se les considera soluciones constantes. La ecuación (2.1) indica que para encontrar dichos puntos, se debe encontrar la solución al siguiente sistema algebraico de ecuaciones:

$$
f\left(u_{0}, v_{0}\right)=0 \quad g\left(u_{0}, v_{0}\right)=0 .
$$


Para una evaluación práctica de estas soluciones estacionarias, se observa que cada una de estas ecuaciones define una curva de pendiente nula (o en inglés null cline). Así, las soluciones estacionarias son la intersección de ambas null clines. Otra utilidad de estas curvas es que proporcionan información del comportamiento global de las trayectorias, pues sabemos que las trayectorias intersectan verticalmente la null cline definida por $f(u, v)=0$, mientras que la otra $g(u, v)=0$ se intersecta horizontalmente.

\section{Perturbaciones y Estabilidad}

En un sistema físico, una perturbación es un agente que influye en su comportamiento de alguna manera no descrita por el modelo matemático. Las perturbaciones pueden ser interiores, lo cual quiere decir que pertenecen inherentemente al sistema que se estudia (como por ejemplo, la inexactitud en las mediciones o la pérdida de información al tomar promedios macroscópicos en un sistema de moléculas), o perturbaciones externas, que sí cambian notablemente el comportamiento del sistema (como por ejemplo cuando una agente externo hace contacto con un péndulo oscilatorio).

Matemáticamente hablando, una perturbación externa puede describirse como un salto en la trayectoria en algún instante $t_{1}$ en el cual empieza la perturbación a otro punto $\left(z_{1}, z_{2}\right)$ :

$$
\left(u\left(t_{1}\right), v\left(t_{1}\right)\right) \longmapsto\left(z_{1}, z_{2}\right),
$$

por lo que cuando la perturbación termina en $t_{2}>t_{1}$ el sistema vuelve a ser gobernado por el sistema de ecuaciones diferenciales, pero con los valores iniciales $u\left(t_{2}\right)=z_{1}$ y $v\left(t_{2}\right)=z_{2}$ de una nueva trayectoria.

La respuesta de un sistema a una perturbación en un punto de equilibrio $\left(u_{0}, v_{0}\right)$ es lo que define su tipo de estabilidad . En ODE's, como en Mecánica, un estado del sistema es estable cuando al perturbar levemente una solución estacionaria, el sistema regresa a su estado original (como tocar una canica en el fondo de un tazón), mientras que el estado es inestable si una perturbación, por pequeña que sea, saca al sistema del estado estacionario en que se encontraba (como un vagón equilibrado en lo más alto de la montaña rusa).

Matemáticamente hablando, una solución estacionaria $\mathbf{w}_{0}$ se dice estable si para condiciones iniciales suficientemente cercanas del punto de equilibrio, la solución tiende a $\mathbf{w}_{0}$ conforme el tiempo avanza, o sea: 


$$
\mathbf{w}(t) \stackrel{t \rightarrow \infty}{\longmapsto} \mathbf{w}_{0} .
$$

A este tipo de puntos se les llama atractores. En contraste, si la solución (o respuesta) se aleja del punto de equilibrio conforme el tiempo avanza, entonces el punto estacionario es inestable.

Hasta ahora no se ha aclarado qué significa estar "suficientemente cerca" de un punto, lo cual define la región de estabilidad o inestabilidad de sistema. En general, dichas regiones son difíciles de obtener, sin embargo, en la práctica conviene estudiar el sistema localmente alrededor de los puntos críticos usando una linearización como se verá a continuación.

\section{Estabilidad lineal}

Una expansión de Taylor de $f$ alrededor de $\left(u_{0}, v_{0}\right)$ da como resultado:

$$
f(u, v) \approx f\left(u_{0}, v_{0}\right)+\frac{\partial f}{\partial u}\left(u_{0}, v_{0}\right)\left(u-u_{0}\right)+\frac{\partial f}{\partial v}\left(u_{0}, v_{0}\right)\left(v-v_{0}\right)
$$

despreciando términos de orden cuadrático, y de órdenes mayores en $\left|\mathbf{w}-\mathbf{w}_{0}\right|$. Como $\mathbf{w}_{0}$ es un nodo estacionario, el primer término de esta suma se anula y sólo restan los términos con las derivadas de primer orden. Haciendo un desarrollo análogo para $g$, y usando la matriz Jacobiana $\mathbb{J}$ :

$$
\mathbb{J}=\frac{\partial \mathbf{R}}{\partial \mathbf{w}}\left(\mathbf{w}_{0}\right)=\left(\begin{array}{ll}
\frac{\partial f}{\partial u} & \frac{\partial f}{\partial v} \\
\frac{\partial g}{\partial u} & \frac{\partial g}{\partial v}
\end{array}\right)_{\left(u_{0}, v_{0}\right)},
$$

con todas las derivadas evaluadas en el punto de equilibrio, el sistema $\dot{\mathbf{w}}=$ $\mathbf{R}(\mathbf{w})$ se puede reemplazar localmente por el sistema lineal:

$$
\frac{d \mathbf{z}}{d t}=\mathbb{Z} \mathbf{z}
$$

donde $\mathbf{z}=\mathbf{w}-\mathbf{w}_{0}$ representa la aproximación a primer orden de puntos cercanos a $\mathbf{w}_{0}$ o, en otras palabras, el comportamiento local de la solución.

De este modo, la cuestión de la estabilidad se reduce al estudio de las soluciones de la ecuación lineal (2.2). El procedimiento estándar para encontrar la solución local es proponer una solución de la forma:

$$
\mathbf{z}(t)=e^{\lambda t} \mathbf{h},
$$


e insertar esta forma en la ecuación diferencial (2.2), resultando en el problema de eigenvalores:

$$
(\mathbb{J}-\lambda \mathbb{I}) \mathbf{h}=0,
$$

siendo $\lambda$ el valor propio, $\mathbf{h}$ el eigenvector e II la matriz identidad. La existencia de soluciones no triviales requiere que los eigenvalores $\lambda_{1}$ y $\lambda_{2}$ sean las raíces de la ecuación característica:

$$
\mathfrak{d e t}(\mathbb{J}-\lambda \mathbb{I})=0 .
$$

En el problema bidimensional, esto resulta en una ecuación cuadrática cuyas raíces $\lambda_{1}$ y $\lambda_{2}$ son obtenidas fácilmente. Los eigenvectores w se calculan al insertar los valores obtenidos directamente en (2.2) y resolver el sistema de ecuaciones lineales.

De los valores obtenidos para los valores propios, uno puede regresar a la ecuación (2.3) y analizar el tipo de comportamiento de los puntos fijos, ya sean nodos, focos o puntos silla. Para nuestro estudio requerimos solamente del siguiente teorema atribuido a Liapunov:

Teorema Suponga $\mathbf{R}(\mathbf{w})$ una función continua dos veces diferenciable y $\mathbf{R}\left(\mathbf{w}_{0}\right)=0$. Sean $\lambda_{j}, j=1,2 \ldots n$ los eigenvalores de la matriz Jacobiana evaluados en la solución estacionaria. Estos determinan la estabilidad de la siguiente manera:

- Si $\mathfrak{R e}\left(\lambda_{j}\right)<0$ para todo $j$, el punto es estable.

- Si $\mathfrak{R e}\left(\lambda_{k}\right)>0$ para algún $k$, el punto es inestable.

En el caso en que todos o algunos de los eigenvalores sólo tengan parte imaginaria la situación es más complicada, sin embargo, este caso no es de nuestro interés ya que, como se verá en detalle más adelante, la formación de patrones requiere estabilidad lineal del primer modo de Fourier de la solución e inestabilidad en alguno de los modos restantes.

\subsection{Reacción oscilante Belouzov-Zhabotinski}

La reacción BZ es el ejemplo clásico de un oscilador químico no-lineal ${ }^{2}$. Su relevancia radica en mostrar que las reacciones químicas no necesariamente

\footnotetext{
${ }^{2}$ La mayor parte de esta sección se extrajo de las referencias [4, 28].
} 
tienen que estudiarse cerca del equilibrio termodinámico. Por este hecho la reacción es de gran interés en problemas biológicos, donde la mayoría de los procesos ocurren lejos del equilibrio. Otro aspecto interesante de esta reacción es la llamada excitabilidad. Según esta propiedad, bajo la influencia de un estímulo (por ejemplo, la luz) pueden darse patrones espaciales por medio de la actividad auto-organizante de los catalizadores involucrados.

El punto fundamental de la reacción BZ es la competencia entre dos catalizadores, según un proceso que se podría resumir como sigue:

- En la primera reacción, el compuesto X aumenta rápidamente, pues funge como catalizador de él mismo (es decir, lleva a cabo un proceso autocatalítico).

- Sin embargo, tanto como crece la cantidad de X, se van agotando los otros reactantes que permiten la creación de X, puesto que la reacción consume más de lo que ella misma puede proveer.

- La concentración de los reactantes que dan lugar a X caen en picada y en la mezcla abunda solamente los productos de X. Esto torna el sistema de un color, digamos azul.

- Conforme los reactantes desaparecen, el proceso autocatalítico pierde ímpetu, y esto permite que la segunda reacción compita en prioridad.

- Esta nueva reacción genera otro producto Y, que puede iniciar su propio ciclo autocatalítico, y por lo tanto pinta la mezcla de otro color, digamos el rojo.

- Esto consume casi todo el producto dejados por X, dejando a su vez como productos especies que sirven como reactantes para producir X.

- Entonces hay condiciones nuevamente para la creación del catalizador y la mezcla puede reiniciar el proceso de autocatálisis.

- Y así sucesivamente, alternado ciclos de azul y rojo hasta agotar la excitabilidad del medio donde se dé la reacción.

Belousov observó que las concentraciones de ambos catalizadores X y Y suben y bajan alternada y coordinadamente formando un patrón temporal: azul, luego rojo, luego azul, luego rojo, etc., en todo el sistema. No obstante, esta reacción también puede producir patrones espaciales. Si en lugar de 
mezclar todos los compuestos se deja que estos se difundan libremente, entonces pequeñas variaciones locales de uno u otro catalizador producidas por la difusión pueden generar regiones alternadas de un color o de otro. Estas variaciones se propagan como anillos concéntricos o espirales que irradian hacia afuera, como cuando se tira una piedra en el agua.

Para entender porque la propagación ocurre de esta manera cuando no hay mezclado, obsérvese lo siguiente. Conforme el ciclo autocatalítico de X toma lugar en un punto, digamos el origen, su influencia se expande en el medio circundante y la región azul se expande en forma de círculo. Mientras avanza la circunferencia o el frente de onda, la mancha se alimenta agotando los reactantes que producen el azul hasta consumirse. Entonces, cerca del origen surgen condiciones para la preponderancia de Y sobre X. Conforme Y lleva a cabo su propio ciclo, su predominio se traduce en un nuevo círculo rojo concéntrico con el primero hasta que nuevamente se agotan los productos de Y y X puede reiniciar el proceso. El resultado es un conjunto de franjas concéntricas rojas y azules que parecen moverse hacia afuera como ondas viajeras, con una frecuencia fija. Estas formas circulares pueden cambiarse experimentalmente si se introducen perturbaciones mecánicas que rompan las líneas. Por ejemplo, si se rompe el frente de una onda con una aguja, se forman dos espirales que giran en sentidos contrarios.

\section{El Brusselator}

Para estudiar este tipo de reacciones oscilantes se han propuesto diversos modelos de reacción química. Quizá el más sencillo sea el "bruselator", llamado así debido a que fue propuesto en la Universidad de Bruselas por Ilya Prigogine y Lefever en 1968 [29]. El brusselator es un modelo teórico de reacción autocatalítica donde se ha demostrado la existencia de oscilaciones químicas y ondas viajeras como las que se han encontrado en la reacción BZ.

El brusselator está dado por cuatro reacciones elementales:

$$
\begin{array}{rll}
A & \stackrel{k_{1}}{\rightarrow} X, \\
B+X & \stackrel{k_{2}}{\rightarrow} Y+E, \\
2 X+Y & \stackrel{k_{3}}{\rightarrow} & 3 X, \\
X & \stackrel{k_{4}}{\rightarrow} & F,
\end{array}
$$

El esquema neto de la reacción es simplemente:

$$
A+B \rightarrow E+F,
$$


donde $X$ y $Y$ sólo fungen como catalizadores de reacciones intermedias. Si asumimos que los reactantes $A$ y $B$ se mantienen a un valor fijo durante toda la reacción y los productos $E$ y $F$ son removidos en cuanto se forman, entonces, si la solución está bien agitada, la evolución de la concentración de $X$ total puede calcularse como la contribución de su consumición o producción en cada paso elemental. La ley de acción de masas indica que la dinámica de los catalizadores está dada por:

$$
\begin{gathered}
\frac{d[X]}{d t}=k_{1}[A]-k_{2}[B][X]+k_{3}[X]^{2}[Y]-k_{4}[X] \\
\frac{d[Y]}{d t}=k_{2}[B][X]-k_{3}[X]^{2}[Y] .
\end{gathered}
$$

donde los paréntesis cuadrados hacen referencia a la concentración. Este procedimiento nos da las leyes diferenciales de velocidad que deben ser resueltas, es decir, integradas analítica o numéricamente teniendo en cuenta las concentraciones iniciales de cada elemento de la reacción. En términos matemáticos, estas ecuaciones definen un sistema de ecuaciones no lineales muy semejante a las estudiadas por Lotka-volterra para los modelos presapredador [26]. Por ello, ahora repetimos un análisis de estabilidad semejante para estudiar cualitativamente las soluciones del Brusselator.

En este caso $[A]$ y $[B]$ fungen como los parámetros del eigenvalor $\lambda$. Uno puede verificar fácilmente que el punto de equilibrio es:

$$
[X]_{0}=\frac{k_{1}}{k_{4}}[A], \quad[Y]_{0}=\frac{k_{4} k_{2}[B]}{k_{3} k_{1}[A]} .
$$

La matriz jacobiana evaluada en el punto estacionario es:

$$
\mathbb{J}=\left(\begin{array}{cc}
k_{2}[B]-k_{4} & k_{3}[X]_{0}^{2} \\
-k_{2}[B] & -k_{3}[X]_{0}^{2}
\end{array}\right) .
$$

El tipo de estabilidad de este punto depende de las raíces de la ecuación característica $\mathfrak{d e t}(\mathbb{J}-\lambda \mathbb{I})=0$, que se obtienen fácilmente como:

$$
\lambda^{2}-(\mathfrak{t r} \mathbb{J}) \lambda+\mathfrak{d} \mathfrak{e t} \mathbb{J}=0,
$$

es decir

$$
\lambda_{ \pm}=\frac{1}{2}\left(\mathfrak{t r} \mathbb{J} \pm \sqrt{(\mathfrak{t r} \mathbb{J})^{2}-4 \mathfrak{d} \mathfrak{e t} \mathbb{J}}\right) .
$$




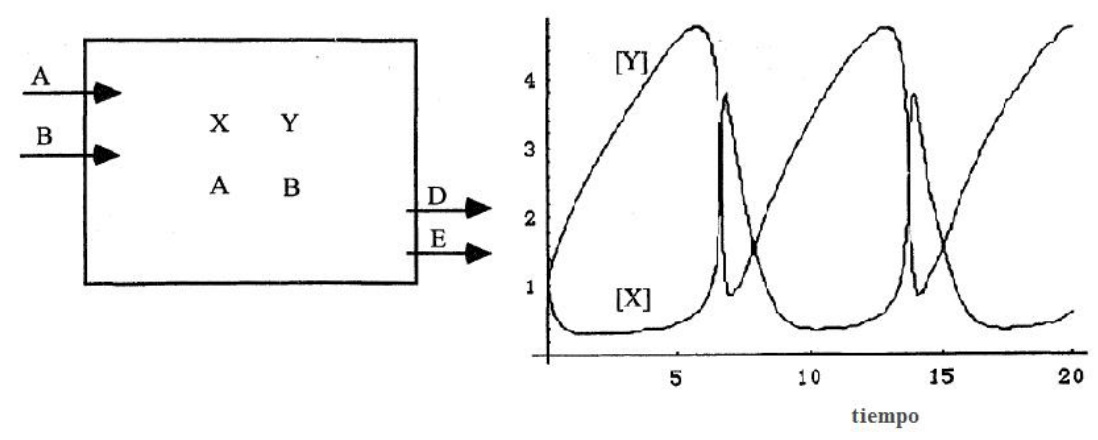

Figura 2.1: Izquierda:Esquema del brusselator. Derecha: oscilaciones típicas del modelo para la reacción BZ.

Nosotros nos concentraremos en las soluciones donde ambos eigenvalores resulten complejos conjugados, pues es en esta región del espacio de parámetros donde se encuentran oscilaciones. Para ello primero buscamos el punto donde la solución pierde su estabilidad en función del incremento en la concetración [B]. Esto ocurre cuando la parte real del eigenvector deviene positiva, es decir, cuando $\mathfrak{t r} \mathbb{J}=k_{2}[B]-k_{4}-k_{3}[X]_{0}^{2}>0$, es decir:

$$
[B]>\frac{k_{4}}{k_{2}}+\frac{k_{3} k_{1}^{2}}{k_{2} k_{4}^{2}}[A]^{2},
$$

en donde se ha sustituido $[X]_{0}$ en términos de $[A]$. Esto quiere decir que, para $[A]$ fijo, la solución es estable hasta que $[B]$ sobrepase este valor. Cuando esto pase, el estado estacionario hace una transición a oscilaciones cuyo tipo depende del valor en el radical de (2.7). Es claro que una combinación de parámetros tal que $(\mathfrak{t r} \mathbb{J})^{2}<4 \mathfrak{d} \mathfrak{e t} \rrbracket$ resulta en eigenvalores con parte imaginaria, y por ende, en oscilaciones en las concentraciones de $[X]$ y $[Y]$. Una de sus oscilaciones típicas se muestra en la Figura 2.1.

La reacción BZ muestra oscilaciones de gran variedad y complejidad, incluso caos. También produce ondas y multiestabilidad. Un gran número de fenómenos interesantes se han estudiado usando esta reacción $[4,6]$. 


\subsection{Difusión}

\section{Leyes de Fick ${ }^{3}$}

La difusión molecular (o simplemente la difusión) se puede describir como el proceso de transporte mediante el cual las moléculas de una región de alta concentración pasan a una región de menor concentración. A grandes rasgos, el resultado de la difusión es un mezcla gradual de los materiales hasta que las concentraciones llegan a ser homogéneas.

Este proceso ha sido estudiado desde la primera mitad del siglo XIX y su enfoque termodinámico moderno puede resumirse en las llamadas leyes de Fick:

Primera ley de Fick. Es una ley fenomenológica que establece que el flujo difusivo va de las regiones de alta concentración a las regiones de baja concentración. La magnitud del flujo es proporcional al gradiente de la concentración, a saber:

$$
\mathbf{J}=-D \nabla w
$$

donde

- J es el vector de flujo de difusión,

- $D$ es el coeficiente de difusión, y

- $w$ es la concentración.

Usualmente la difusión se mide en pares de especies, es decir, depende de cómo una sustancia se difunde dentro de otra sustancia específica; además, en mezclas ideales, el coeficiente de difusión $D$ es proporcional al promedio del cuadrado de la velocidad con que las partículas se difunden, según la relación de Stokes-Einstein.

Segunda ley de Fick. Es una ecuación de balance o conservación de la masa que indica que la concentración local de una especie cambia debido a las moléculas que entran o salen por la difusión. Para formularse matemáticamente, sea $\Omega$ una región en el espacio con superficie de frontera $\Gamma$. El grado

\footnotetext{
${ }^{3}$ La integridad de esta sección es un resumen de [30].
} 
de cambio de masa dentro de la región debe ser igual al flujo que entra o sale por la frontera. Suponiendo que no hay fuentes, se tiene:

$$
\frac{d}{d t} \int_{\Omega} w d \Omega=\int_{\Gamma} \mathbf{J} \cdot \hat{n} d \Gamma .
$$

Sustituyendo la primera ley de Fick en el término de la derecha, suponiendo además que la región no cambia con el tiempo y aplicando el teorema de la divergencia, se obtiene:

$$
\int_{\Omega} \frac{\partial w}{\partial t} d \Omega=\int_{\Omega} \nabla \cdot(D \nabla w) d \Omega .
$$

Como esta relación es válida para cualquier región $\Omega$ y las derivadas de $w$ son continuas, entonces se puede obtener la forma diferencial de esta ley como:

$$
\frac{\partial w}{\partial t}=\nabla \cdot(D \nabla w),
$$

cuya forma matemática es la de una ecuación del tipo parabólico, y que también modela los procesos de difusión de calor, entre otros fenómenos.

\section{La ecuación de Reacción Difusión}

En el caso de que haya reacciones químicas por los cuales se active o inhiba la aparición de la sustancia en cuestión, es necesario añadir a la ecuación de balance de masas un término que describa este proceso, en la siguiente forma:

$$
\frac{d}{d t} \int_{\Omega} w d \Omega=\int_{\Omega} R d \Omega-\int_{\Gamma} \mathbf{J} \cdot \vec{n} d \Gamma,
$$

donde $R(w, \mathbf{x}, t)$ es una función que expresa cómo se desarrolla la cinética química de la mezcla en función de la concentración, la posición y el tiempo.

Por un cálculo análogo al anterior, se puede derivar una ecuación diferencial para un proceso de reacción difusión:

$$
\frac{\partial w}{\partial t}=R+\nabla \cdot(D \nabla w) .
$$

Esta ecuación puede generalizarse para el caso en que existan diversas especies interactuando. Si $w_{i}(\mathbf{x}, t)$ es la concentración de la i-ésima especie (con $i=1,2, \ldots m)$ difundiéndose dentro de las otras con coeficiente $D_{i}, \mathrm{y}$ 
$\mathbf{R}$ es el vector que describe las interacciones entre las $m$ especies, entonces la ecuación de reacción difusión que resulta es:

$$
\frac{\partial \mathbf{w}}{\partial t}=\mathbf{R}(\mathbf{w})+\nabla \cdot(\mathbb{D} \nabla \mathbf{w})
$$

donde ahora $\mathbb{D}$ es una matriz de difusividades, cuya estructura es diagonal a menos que exista algún tipo de difusión cruzada, es decir, efectos anisotrópicos. Suponiendo que esta matriz es constante, la ecuación reacción difusión es simplemente:

$$
\frac{\partial \mathbf{w}}{\partial t}=\mathbf{R}(\mathbf{w})+\mathbb{D} \nabla^{2} \mathbf{w} .
$$

Una ecuación como esta es la que utilizo Turing para describir la formación de patrones estacionarios. 


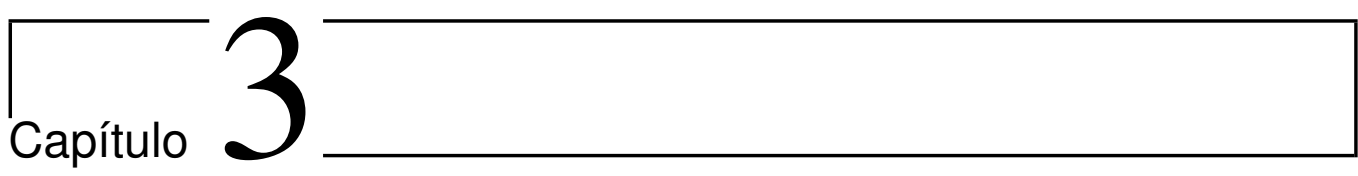

\section{Patrones de Turing}

En este capítulo realizamos el análisis matemático de las ideas de Turing para demostrar la existencia de patrones espaciales estacionarios derivables de un mecanismo $\mathrm{RD}$. Al estudiar la relación de dispersión, la cual define la presencia y el tipo de las oscilaciones, definimos las condiciones del espacio de parámetros. Finalmente, para concretar estas ideas generales, ejemplificamos con la reacción de Schnakenberg.

\subsection{Condiciones matemáticas para la apari- ción de patrones ${ }^{1}$}

Como vimos en el capítulo anterior, la ecuación que describe un mecanismo de reacción difusión está dadas por:

$$
\frac{\partial \mathbf{w}}{\partial t}(\mathbf{x}, t)=\mathbf{R}(\mathbf{w})+\mathbb{D} \nabla^{2} \mathbf{w}
$$

expresión vectorial que en realidad recoge tantas ecuaciones como especies interactuando existan en la mezcla. En nuestro caso, trabajaremos únicamente con dos especies, por lo que $\mathbf{w}$ representa un vector con las dos concentraciones a estudiar, $\mathbf{R}$ expresa la cinética química entre ambas especies y $\mathbb{D}$ es una matriz diagonal con los coeficientes de difusión. Por lo tanto en este capítulo buscaremos las condiciones para que en el sistema:

\footnotetext{
${ }^{1}$ Para mayor información sobre este estudio se pueden consultar las referencias [31, 32].
} 


$$
\begin{aligned}
& \frac{\partial u}{\partial t}=f(u, v)+D_{u} \nabla^{2} u \\
& \frac{\partial v}{\partial t}=g(u, v)+D_{v} \nabla^{2} v
\end{aligned}
$$

aparezcan estructuras de Turing. Antes de empezar a realizar los cálculos conviene señalar que, formalmente hablando, las estructuras de Turing son inhomogeneidades espaciales u oscilaciones que aparecen en el estado estacionario de una mezcla como el resultado, no de los mecanismo químicos, sino de la difusión ${ }^{2}$.

Antes de entrar en la laboriosa tarea del análisis y cálculo de las condiciones matemáticas que hacen posibles la aparición de estos patrones, consideramos necesario bosquejar un camino a seguir:

1. Usaremos una primera condición para la aparición de los patrones, a saber, que en ausencia de difusión, el estado estacionario resulta únicamente de la cinética química del sistema; tal estado debe ser estable a pequeñas perturbaciones. De este modo, la estabilidad del primer modo de Fourier garantizará que la solución (el patrón) sea estacionaria.

2. Usaremos una segunda condición que garantice el hecho de que la difusión sea la causante de oscilaciones espaciales, a saber, que en presencia de la difusión, algunos modos de oscilación del sistema deben ser inestable a pequeñas perturbaciones espaciales. Esto significa que estos modos no se desvanecerán con el tiempo y que serán los causantes de los patrones espaciales.

3. Finalmente hablaremos de la relación de dispersión que establece condiciones entre el desarrollo temporal del proceso y los números de onda requeridos para la aparición de patrones de Turing.

Para que el sistema (3.2) tenga solución bien determinada son necesarias condiciones de frontera e iniciales. Con fines de simplificar la exposición usaremos las siguientes condiciones:

\footnotetext{
${ }^{2}$ Esto resulta muy particular puesto que la difusión usualmente es un proceso estabilizador, además de que las oscilaciones químicas conocidas más estudiadas están relacionadas más bien a la parte temporal.
} 


$$
\begin{array}{r}
\mathbf{n} \cdot \nabla u=0 \text { y } \mathbf{n} \cdot \nabla v=0, \text { para puntos en la frontera } \Gamma, \mathrm{y} \\
u(\mathbf{x}, t=0) \text { y } v(\mathbf{x}, t=0) \text { son concentraciones iniciales dadas. }
\end{array}
$$

En la ecuación refhect1, $\mathbf{n}$ es el vector normal unitario exterior al dominio, y depende del punto escogido sobre $\Gamma$. La razón de escoger condiciones de flujo nulo en la frontera es porque estamos interesados en la auto-organización del sistema para la formación de patrones y descartar el hecho de que sean generados por influencias externas al sistema.

\section{Estabilidad en ausencia de difusión}

\section{En ausencia de difusión, el estado estacionario es estable ante pequeñas perturbaciones.}

Para mostrar esto, empezaremos con un sistema bien mezclado entre los dos componentes. En este caso la difusión no toma parte en el proceso y el sistema es solamente:

$$
\begin{aligned}
& u_{t}=f(u, v), \\
& v_{t}=g(u, v),
\end{aligned}
$$

Evidentemente, la solución de equilibrio se alcanzará cuando

$$
\begin{gathered}
f\left(u_{0}, v_{0}\right)=0, \\
g\left(u_{0}, v_{0}\right)=0 .
\end{gathered}
$$

Sin embargo, supongamos que en algún momento previo detenemos el proceso de mezclado. En este caso, el estado estacionario dependerá ahora de la difusión y estará dado por la solución del sistema:

$$
\begin{gathered}
f(u, v)+D_{u} \nabla^{2} u=0, \\
g(u, v)+D_{v} \nabla^{2} v=0,
\end{gathered}
$$

que dependerá ahora de la posición y de los parámetros $D_{u}$ y $D_{v}$. Sin embargo, las soluciones de equilibrio de (3.6) pueden ser aún soluciones de (3.7), 
siempre y cuando el Laplaciano tanto de $u$ como de $v$ se anulen en el equilibrio, es decir, cuando las soluciones en equilibrio son también funciones armónicas. En otras palabras, estamos interesados en los procesos en los que la difusión no afecte la existencia y particularidad de los estados estacionarios de la reacción química, pero que sí afecte su estabilidad. En particular, buscamos que el estado $\left(u_{0}, v_{0}\right)$, al activar la difusión, cambie a inestable (hasta primer orden) ante perturbaciones espaciales no uniformes, lo cual se estudiará en detalle hasta la próxima sección.

De momento, regresemos al par de ecuaciones (3.5) para ver qué condiciones se pueden imponer para la existencia de patrones. Para simplificar la deducción, sea $\mathbf{w}$ el vector formado por las concentraciones $u$ y $v$. Entonces el sistema (3.5) puede escribirse como:

$$
\dot{\mathbf{w}}=\mathbf{R}(\mathbf{w}),
$$

con $\mathbf{R}=(f(u, v), g(u, v))$ la función de las cinéticas. Sea $\mathbf{w}_{0}$ la solución de equilibrio obtenida de resolver (3.6). Sea además $\mathbb{J}$ la matriz Jacobiana con las primeras derivadas de $f$ y $g$ evaluadas en este punto:

$$
\mathbb{J}=\left(\begin{array}{ll}
f_{u} & f_{v} \\
g_{u} & g_{v}
\end{array}\right)_{(u 0, v 0)} .
$$

Expandiendo la función $\mathbf{R}$ alrededor de este punto hasta el primer orden, se obtiene la aproximación $\mathbf{R}(\mathbf{w}) \approx \mathbf{R}\left(\mathbf{w}_{0}\right)+\mathbb{J} \cdot\left(\mathbf{w}-\mathbf{w}_{0}\right)$, donde el primer término se anula por ser $\mathbf{w}_{0}$ solución de equilibrio. La ecuación anterior puede escribirse entonces simplemente como:

$$
\dot{\mathbf{z}}=\mathbb{J} \mathbf{z}
$$

con $\mathbf{z}=\mathbf{w}-\mathbf{w}_{0}$. Proponiendo una solución de la forma $\mathbf{z}=e^{\mu t} \mathbf{h}$ y sustituyendo en la ecuación anterior, se obtiene el siguiente problema de eigenvalores: $(\mathbb{J}-\lambda \mathbb{I}) \mathbf{h}=0$, que tiene solución no trivial cuando el determinante del término entre paréntesis sea cero. Los dos eigenvalores para este problema están dados por:

$$
\lambda_{ \pm}=\frac{1}{2}\left[\mathfrak{t r} \mathbb{J} \pm \sqrt{\mathfrak{t r}^{2} \mathbb{J}-4 \cdot \mathfrak{d} \mathfrak{e t} \mathbb{J}}\right]
$$

siendo $\mathfrak{t r} \mathbb{J}$ y $\mathfrak{d e t} \mathbb{J}$ la traza y el determinante de la matriz Jacobiana respectivamente. Recordamos ahora que en este primer paso requerimos que los 
puntos de equilibrio ( necesita que $\mathfrak{R e} \lambda<0$, lo cual resulta en las condiciones:

$$
\begin{aligned}
\mathfrak{t} \mathfrak{} \mathbb{J} & <0, \quad \mathrm{y} \\
\mathfrak{d} \mathfrak{e t} \mathbb{J} & >0 .
\end{aligned}
$$

las cuales constituyen las primeras dos condiciones que buscamos.

\section{Inestabilidad espacial ante la presencia de la difusión}

En presencia de la difusión el sistema linearizado es inestable ante pequeñas perturbaciones espaciales

Se requiere que la difusión cause un cambio, no en las concentraciones de equilibrio, sino más bien en su estabilidad. Lo cual quiere decir que debe tener algunos efectos que no desaparezcan con el tiempo; en particular, se requiere la formación de oscilaciones espaciales en las concentraciones tal que puedan generar un patrón. Algo importante es que esta inestabilidad ocurra solamente para el sistema lineal: la presencia de términos de la solución lineal que crecen con el tiempo (y que posibilitan la formación de patrones) no significa que las concentraciones crecerán infinitamente con el tiempo sino que sus efectos serán balanceados por los términos de orden más alto para lograr la estabilización de toda la solución, como se verá al final de la sección. Por ende, la condición de inestabilidad lineal es un artilugio para poder estudiar analíticamente condiciones en el sistema y concentrar los efectos espaciales en el término lineal.

Una vez asentado esto, consideremos el sistema completo de reacción difusión dado por ecuación (3.2), que en forma vectorial puede escribirse como:

$$
\mathbf{w}_{t}=\mathbf{R}(\mathbf{w})+\mathbb{D} \nabla^{2} \mathbf{w}
$$

con $\mathbf{R}$ definida igual que antes y la matriz de difusión $\mathbb{D}$ dada por:

$$
\mathbb{D}=\left(\begin{array}{cc}
D_{u} & 0 \\
0 & D_{v}
\end{array}\right) .
$$

Pero antes de poder plantear el problema de eigenvalores para esta ecuación que depende no solo del tiempo, sino también del espacio, es conveniente 
resolver el problema de valores propios, que denotaremos por $k$, para el operador Laplaciano, los cuales son independientes del tiempo y satisfacen:

$$
\nabla^{2} \mathbf{W}(\mathbf{r})+k^{2} \mathbf{W}(\mathbf{r})=\overrightarrow{0}
$$

con condiciones de flujo nulo $(\hat{\mathbf{n}} \cdot \nabla) \mathbf{W}=\overrightarrow{0}$ en la frontera. La solución de este problema depende única y exclusivamente de la geometría del sistema. Para dominios acotados, $k$ toma usualmente valores discretos, los cuales se pueden numerar. Las soluciones de esta ecuación son generalmente funciones oscilatorias y $k$ representa el modo de oscilación de la solución, por ello es llamado número de onda.

Suponiendo que se ha resuelto la ecuación (3.10), lo cual significa también haber encontrado el conjunto posible de valores de $k$, se puede ahora proponer una solución para la ecuación (3.9) en la forma:

$$
\mathbf{w}(\mathbf{r}, t)=\sum_{k} c_{k} e^{\lambda_{k} t} \mathbf{W}_{k}(\mathbf{r})
$$

donde $\mathbf{W}_{k}(\mathbf{r})$ son las soluciones del problema espacial (3.10). Por construcción, estas funciones satisfacen automáticamente las mismas condiciones de frontera de flujo nulo del problema original. Al sustituir esta solución linealizada en la ecuación (3.9), se obtiene que, para cada eigenvalor k:

$$
\left(\lambda_{k} \mathbb{I}-\mathbb{J}+k^{2} \mathbb{D}\right) \mathbf{w}=0 .
$$

Este problema tiene solución no trivial cuando la matriz entre paréntesis tiene determinante cero. Esto resulta en un polinomio característico de segundo grado para $\lambda$ que, mediante un poco de álgebra, resulta ser:

$$
\begin{array}{r}
\lambda_{k}^{2}+\lambda_{k}\left[k^{2} \mathfrak{t r} \mathbb{D}-\mathfrak{t r} \mathbb{J}\right]+h\left(k^{2}\right)=0, \\
\operatorname{con} h\left(k^{2}\right)=(\mathfrak{d} \mathfrak{e t} \mathbb{D}) k^{4}-(\mathbb{D}: \mathbb{J}) k^{2}+\mathfrak{d} \mathfrak{e t} \mathbb{J},
\end{array}
$$

donde $\mathbb{D}: \mathbb{J}=D_{v} f_{u}+D_{u} g_{v}$. En la sección anterior hemos visto las condiciones para que el modo $k=0$ alcance un estado estable en ausencia de difusión. Para este modo, los efectos espaciales están dados por la ecuación $\nabla^{2} \mathbf{W}(\mathbf{r})=0$ y las condiciones (3.8) que, como se recordará, estas últimas se obtuvieron pidiendo $\mathfrak{R e} \lambda_{k=0}<0$. Ahora, para que existan modos inestables a perturbaciones espaciales, es preciso pedir que $\mathfrak{R e} \lambda_{k}>0$ para algún $k$. A esta relación entre $\lambda$ y $k$ se le conoce como relación de dispersión. El análisis 
detallado de esta relación requiere de cuidado por lo que iniciamos su estudio un poco más abajo.

De momento, solo resaltamos que además de las condiciones (3.8), el estudio de (3.13) indica que la inestabilidad lineal en presencia de difusión implica otro par de condiciones(lo cual será demostrado a continuación), a saber:

$$
\begin{aligned}
&(\mathbb{D}: \mathbb{J})^{2}-4(\mathfrak{d} \mathfrak{e t} \mathbb{D}) \mathfrak{d e t} \mathbb{J}>0 \\
& \mathbb{D}: \mathbb{J}>0 .
\end{aligned}
$$

\section{La relación de dispersión}

A continuación analizaremos la relación de dispersión (3.13) para obtener las condiciones que permitan $\mathfrak{R e} \lambda_{k}>0$ para algún $k$. Puesto que $\mathfrak{t r} \mathbb{J}<0$ por (3.8) y $k^{2} \mathfrak{t r} \mathbb{D}>0$ para toda $k \neq 0$, entonces $\left[k^{2} \mathfrak{t r} \mathbb{D}-\mathfrak{t r} \mathbb{J}\right]>0$. Por ende, la única forma de que $\mathfrak{R e} \lambda_{k}>0$ para algún $k$ es que el término $h\left(k^{2}\right)<0$, lo cual es claro de las soluciones del polinomio para $\lambda$ :

$$
\lambda_{k}=\frac{1}{2}\left\{\left[\mathfrak{t r} \mathbb{J}-k^{2} \mathfrak{t r} \mathbb{D}\right] \pm\left\{\left[k^{2} \mathfrak{t r} \mathbb{D}-\mathfrak{t r} \mathbb{J}\right]^{2}-4 h\left(k^{2}\right)\right\}^{1 / 2}\right\} .
$$

Volviendo sobre (3.13) y recordando que teníamos $\mathfrak{d e t} \mathbb{J}>0$ por (3.8), debe tenerse entonces $\mathbb{D}: \mathbb{J}>0$ lo cual es la segunda condición en (3.14). Esta desigualdad es necesaria, pero no suficiente. Para que $h\left(k^{2}\right)$ sea negativo para algún valor de $k$, es necesario además que el mínimo de la función $h$ sea negativo. Diferenciando esta función respecto a $k^{2}$ de (3.13), se obtiene que el mínimo de la función se toma en:

$$
k_{\min }^{2}=\frac{\mathbb{D}: \mathbb{J}}{2 \mathfrak{d} \mathfrak{e t} \mathbb{D}}
$$

donde la función vale

$$
h\left(k_{\text {min }}^{2}\right)=\left[\mathfrak{d} \mathfrak{e t} \mathbb{J}-\frac{(\mathbb{D}: \mathbb{J})^{2}}{4 \mathfrak{d} \mathfrak{e t} \mathbb{D}}\right] .
$$

De donde resulta claro que $h_{\text {min }}$ será negativo si el término en el paréntesis cuadrado es negativo, obteniendo la primera condición de (3.14). 
Ahora nos concentramos en la función $h\left(k^{2}\right)$ en (3.13):

$$
h\left(k^{2}\right)=(\mathfrak{d} \mathfrak{e} \mathbb{D}) k^{4}-(\mathbb{D}: \mathbb{J}) k^{2}+\mathfrak{d} \mathfrak{e t} \mathbb{J} .
$$

Esta es una función cuadrática (en $k^{2}$ ) y convexa por las condiciones que acabamos de analizar. Su mínimo se encuentra en $k_{\min }^{2}$ y sus valores son negativos en el rango que está entre las raíces de $h\left(k^{2}\right)$ :

$$
k_{1}^{2}, k_{2}^{2}=\frac{\gamma}{2 \mathfrak{d} \mathfrak{e} \mathbb{D}}\left[(\mathbb{D}: \mathbb{J}) \pm\left[(\mathbb{D}: \mathbb{J})^{2}-4(\mathfrak{d e t} \mathbb{D}) \mathfrak{d} \mathfrak{e t} \mathbb{J}\right]^{1 / 2}\right]
$$

Esto quiere decir que solo los modos con números de onda $k$ contenido entre estos dos valores son aquellos que producen patrones espaciales. Todos aquellos que no se encuentre en este rango tienen un decaimiento exponencial en el tiempo y se desvanecen, ver Figura 3.1. Por ello, volviendo a la ecuación (3.11), para tiempos suficientemente largos la solución a primer orden es simplemente:

$$
\mathbf{w}(\mathbf{r}, t)=\sum_{k_{1}}^{k_{2}} c_{k} e^{\lambda_{k} t} \mathbf{W}_{k}(\mathbf{r})
$$

El supuesto de esta solución es que las soluciones linealmente inestables que crecen exponencialmente con el tiempo, gradualmente se van acotando con los términos no lineales de las ecuaciones RD, para que al final emerja una solución estacionaria con homogeneidades espaciales. El elemento clave para sustentar esta suposición es que el sistema es espacialmente acotado. Uno esperaría que si un conjunto de soluciones existen únicamente para la cinética química, el mismo conjunto contendrá las soluciones cuando la difusión es incluida, lo cual ya ha sido rigurosamente demostrado [32]

\subsection{Ejemplo: reacción de Schnakenberg}

\section{Cinética}

Una de las reacciones oscilantes más simples entre dos especies químicas fue sugerida por Schnakenberg en 1979 [43]. Su mecanismo es el siguiente:

$$
X \underset{k_{4}}{\stackrel{k_{1}}{\leftrightarrows}} A, \quad B \stackrel{k_{2}}{\longrightarrow} Y, \quad 2 X+Y \stackrel{k_{3}}{\longrightarrow} 3 X .
$$




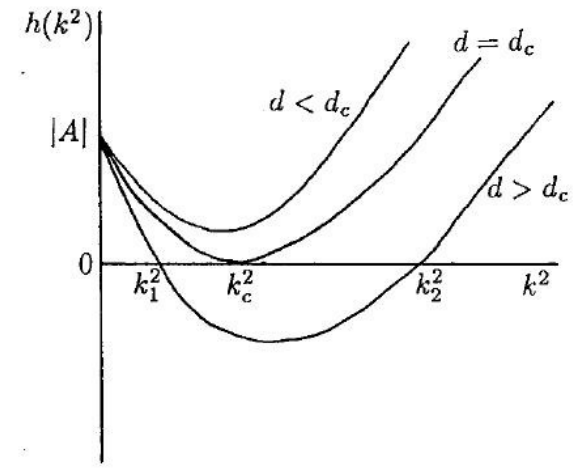

(a)

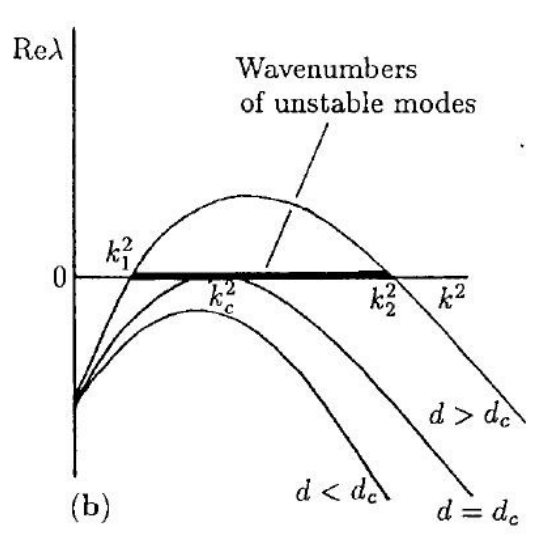

Figura 3.1: Gráfica de $h\left(k^{2}\right)$ definido por 3.15 para una cinética típica. Cuando la razón entre ambos coeficientes de difusión $d=D_{v} / D_{u}$ alcanza un valor crítico $d_{c}$, entonces $h\left(k^{2}\right)$ se torna negativo en un rabgo finito de $k^{2}$. (b) Gráfica de los eigenvalores más grandes de $\lambda$ como función de $k^{2}$. Cuando $d>d_{c}$ hay un rango de números de onda $k_{1}^{2}<k^{2}<k_{2}^{2} t$ que son linealmente inestables. Tomado de ([32]).

Al usar la ley de acción de masas, se obtiene que la cinética de los catalizadores A y B está dada por:

$$
\begin{gathered}
F(A, B)=k_{1}-k_{2} A+k_{3} A^{2} B \\
G(A, B)=k_{4}-k_{3} A^{2} B .
\end{gathered}
$$

Este modelo explica el comportamiento de un químico activador A en presencia de un inhibidor B. El término $A^{2} B$ es tal que en la primera ecuación representa la producción de $\mathrm{A}$ en presencia de $\mathrm{B}$, mientras que en la segunda ecuación representa el consumo de $\mathrm{B}$ en presencia de A. El termino proporcional a $-A$ representa la degradación o reducción del mismo en la primera ecuación, produciendo así un aumento de B en la segunda reacción [42]. Por ello, a este tipo de reacciones se les llama de reducción de sustrato (substrate-depletion en inglés) La reacción se puede esquematizar como en la Figura (3.2): 


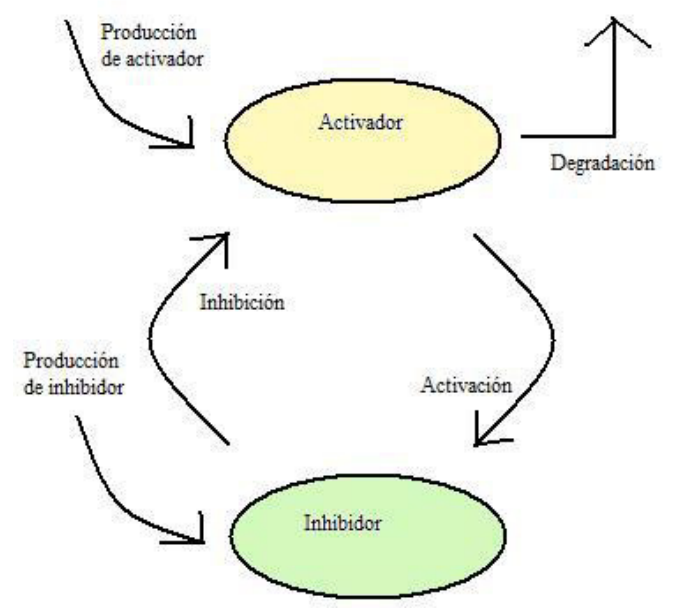

Figura 3.2: Esquema para la reacción de Schnakenberg.

\section{Adimensionalización}

Sean $t, \mathbf{x}$, el tiempo y la posición de un punto en el dominio, respectivamente, entonces los valores adimensionales de $t, x, A, B$, se definen por las expresiones:

$$
t^{*}=\frac{D_{A} t}{L^{2}}, \mathbf{x}^{*}=\frac{\mathbf{x}}{L}, u=A\left(\frac{k_{3}}{k_{2}}\right)^{1 / 2}, v=B\left(\frac{k_{3}}{k_{2}}\right)^{1 / 2} .
$$

Respectivamente, en donde $L$ es el tamaño característico del dominio, $D_{A}$ y $D_{B}$ los coeficientes de difusión de las sustancias. Para simplificar, definimos además las constantes $d, \gamma, a$ y $b$ de la siguiente manera:

$$
d=\frac{D_{B}}{D_{A}}, \gamma=\frac{L^{2}}{D_{A}} k_{2}, a=\frac{k_{1}}{k_{2}}\left(\frac{k_{3}}{k_{2}}\right)^{1 / 2}, b=\frac{k_{4}}{k_{2}}\left(\frac{k_{3}}{k_{2}}\right)^{1 / 2}
$$

respectivamente. Hecho lo anterior, el sistema adimensional que resulta para la reacción de Schnakenberg se puede escribir de la siguiente forma:

$$
\begin{aligned}
& u_{t}=\gamma\left(a-u+u^{2} v\right)+\nabla^{2} u \\
& v_{t}=\gamma\left(b-u^{2} v\right)+d \nabla^{2} v
\end{aligned}
$$


Basta observar la adimensionalización (3.18) para notar que los parámetros $a, b, d$ y $\gamma$ deben ser positivos si pretenden modelar una reacción real. Esto se debe a que los coeficientes de difusión y las velocidades de reacción son positivas en este caso.

\section{Condiciones para la aparición de patrones}

El punto de equilibrio estable de la reacción química:

$$
\begin{gathered}
f(u, v)=\gamma\left(a-u+u^{2} v\right), \\
g(u, v)=\gamma\left(b-u^{2} v\right) .
\end{gathered}
$$

se encuentra resolviendo $f=0, g=0$, encontrando fácilmente que:

$$
\begin{gathered}
u_{0}=a+b, \\
v_{0}=\frac{b}{(a+b)^{2}} .
\end{gathered}
$$

Al sustituir estos valores y teniendo en cuenta que todos los parámetros son positivos en las diversas derivadas se obtiene:

$$
\begin{gathered}
f_{u}=\gamma \frac{b-a}{a+b}, \quad f_{v}=\gamma(a+b)^{2}>0 \\
g_{u}=\gamma \frac{-2 b}{a+b}<0, \quad g_{v}=-\gamma(a+b)^{2}<0,
\end{gathered}
$$

y el determinante del Jacobiano es $f_{u} g_{v}-f_{v} g_{u}=\gamma^{2}(a+b)^{2}>0$. Con estas expresiones, las condiciones encontradas anteriormente para la formación de patrones de Turing (3.8 y 3.14), se traducen en las siguientes cuatro desigualdades:

$$
\begin{aligned}
f_{u}+g_{v}<0 & \Rightarrow 0<b-a<(a+b)^{3}, \\
f_{u} g_{v}-f_{v} g_{u}>0 & \Rightarrow(a+b)^{2}>0 \\
d f_{u}+g_{v}>0 & \Rightarrow d(b-a)>(a+b)^{2}>0 \\
\left(d f_{u}+g_{v}\right)^{2}-4 d\left(f_{u} g_{v}-f_{v} g_{u}\right)>0 & \Rightarrow\left[d(b-a)-(a+b)^{3}\right]^{2}>4 d(a+b)^{3} .
\end{aligned}
$$




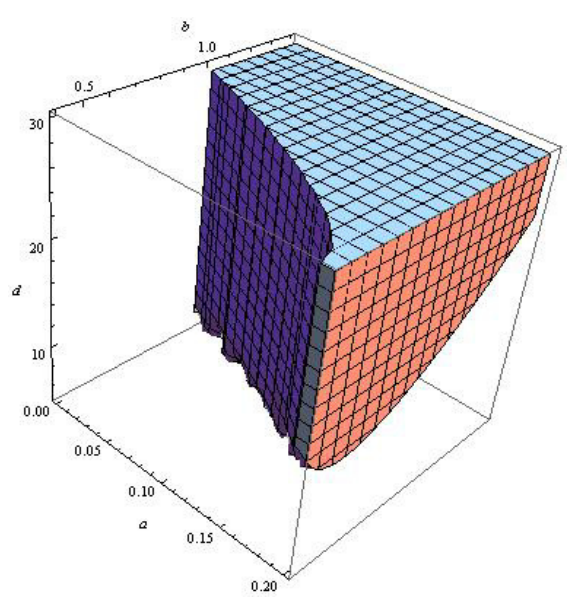

Figura 3.3: Espacio de Turing de la reacción de Schnakenberg

donde se ha usado que $b>a$ de la tercera condición para sustituirlo en la primera. En el espacio $(a, b, d)$ esto define una región de valores posibles para la existencia de patrones. Este dominio es llamado espacio de Turing y sirve para determinar bajo qué perturbaciones espaciales el sistema será inestable en presencia de difusión. Ver Figura 3.3.

\section{Inhomogeneidades espaciales}

Para ver como se producen las inhomogeneidades, considere el problema espacial de eigenvalores (3.10), escogiendo el caso unidimensional en el dominio definido por $x \in[0, p]$ :

$$
\nabla^{2} \mathbf{W}(x)+k^{2} \mathbf{W}(x)=0
$$

con condiciones de flujo nulo en la frontera definida por $x=0$ y $x=p$. Las soluciones del problema son de la forma:

$$
\mathbf{W}_{n}(x)=\mathbf{A}_{n} \cos \left(\frac{n \pi x}{p}\right), n= \pm 1 \pm 2, \ldots
$$

con $\mathbf{A}_{n}$ con constantes arbitrarias. Los valores propios son el conjunto discreto de números de onda dados por:

$$
k_{n}=\left(\frac{n \pi}{p}\right) .
$$


Por tanto, una vez seleccionados los parámetros $a, b$ y $d$, debe elegirse $\gamma$ de tal manera que el número de onda $k$ esté entre los valores definidos por (3.16):

$$
\gamma L(a, b, d)=k_{1}^{2}<k_{n}^{2}<k_{2}^{2}=\gamma M(a, b, d)
$$

con $L$ y $M$ dados por:

$$
\begin{aligned}
& L=\frac{\left[d(b-a)-(a+b)^{3}\right]-\left\{\left[d(b-a)-(a+b)^{3}\right]^{2}-4 d(a+b)^{4}\right\}}{2 d(a+b)}, \\
& M=\frac{\left[d(b-a)-(a+b)^{3}\right]+\left\{\left[d(b-a)-(a+b)^{3}\right]^{2}-4 d(a+b)^{4}\right\}}{2 d(a+b)} .
\end{aligned}
$$

En otras palabras, todos los modos de la solución se desvanecerán con el tiempo a menos que exista al menos algún entero $n$ tal que:

$$
\gamma \frac{p^{2} L(a, b, d)}{\pi^{2}}<n^{2}<\gamma \frac{p^{2} M(a, b, d)}{\pi^{2}} .
$$

Esto significa que habrá patrones de Turing sólo en el caso de que los parámetros y el tamaño del dominio permitan la existencia de modos de onda $k_{n}$ tales que 1) quepan en la región, y 2) no se desvanezcan por el efecto homogeneizador de la difusión. Ver Figura 3.4. 


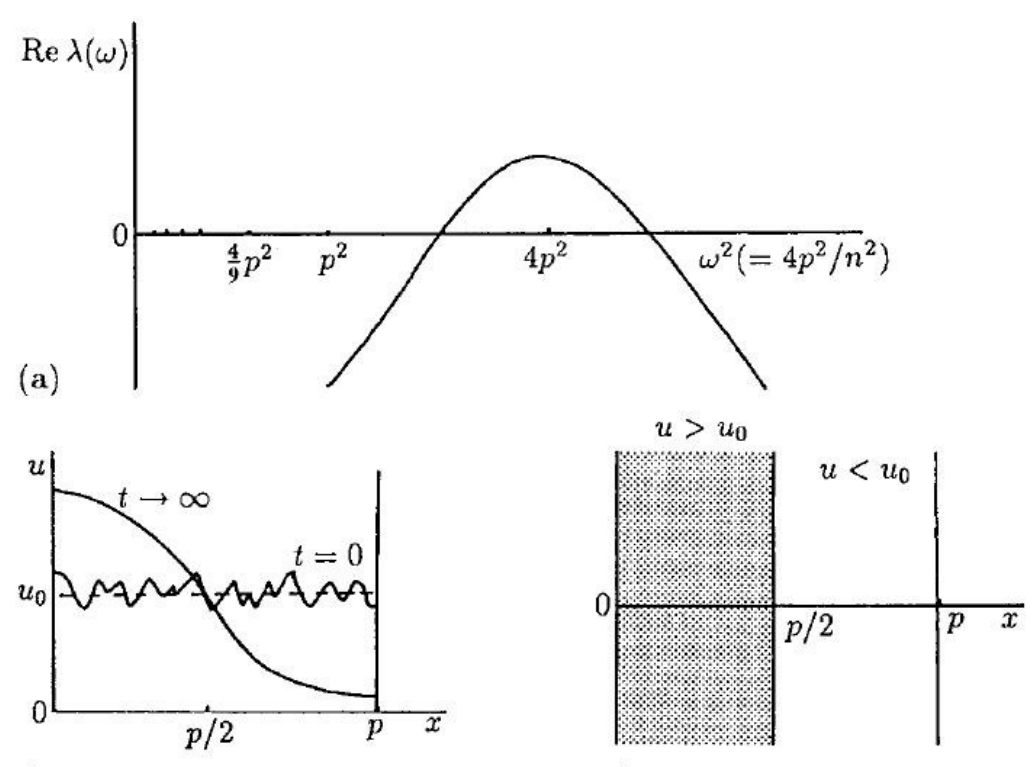

(b)

(c)

Figura 3.4: (a)Relación de dispersión típica para $\mathfrak{R e}(\lambda)$ como función de la longitud de onda $\omega=2 \pi / k$ obtenida de la linearización alrededor del punto de equilibrio. En este ejemplo el único modo inestable es $n=1$; todos los demás tienen $\mathfrak{R e}(\lambda)<0$. (b) El desenvolvimiento temporal desde condiciones iniciales aleatorias hasta patrones espaciales de amplitud finita. (c) Patrón obtenido, donde el área sombreada corresponde a regiones de alta concentración, y las blancas a zonas de menor concentración a las del estado estacionario. Tomado de [32]. 
$\Gamma_{\text {Capítulo }}$

\section{Métodos numéricos}

Como se vió en el capítulo anterior, el análisis lineal de las ecuaciones RD nos garantiza la existencia de patrones dada cierta configuración de parámetros. Sin embargo, dicho análisis no nos dice el tipo de patrón que se obtendrá, ni como este dependerá de las condiciones iniciales, tampoco nos indica qué efectos puedan tener los términos no lineales. Además, usualmente no es posible encontrar soluciones analíticas para este tipo de ecuaciones diferenciales no lineales. Por lo tanto, para hacer un análisis más exhaustivo, además de obtener la forma y características de algunos patrones conviene resolver las ecuaciones RD numéricamente, por lo que ahora resumimos dos de la estrategias de discretización más comunes para la resolución de este tipo de ecuaciones, a saber los métodos de diferencia finitas y elemento finito.

\section{Planteamiento del problema y notación}

Nos proponemos resolver el sistema de ecuaciones reacción difusión para el caso de dos especies químicas $u$ y $v$ resumidas en el vector $\mathbf{w}$. Las interacciones químicas están dadas por el vector $\mathbf{R}(\mathbf{w})$ y las difusividades de cada compuesto se suponen constantes y ubicadas en la traza de la matriz de difusividades $\mathbb{D}$. Vectorialmente, la ecuación a resolver es:

$$
\frac{\partial \mathbf{w}}{\partial t}(\mathbf{x}, t)=\mathbf{R}(\mathbf{w})+\mathbb{D} \nabla^{2} \mathbf{w}
$$


Sólo con fines de simplificar la exposición ${ }^{1}$, la deducción de los métodos se hará para el caso en que el dominio $\Omega$ es un cuadrado de lado $p$ y con condiciones de flujo nulo en la frontera $\Gamma$, es decir:

$$
\nabla \mathbf{w}(t) \cdot \hat{\mathbf{n}}=\overrightarrow{0} \text { para } \mathbf{x} \in \Gamma .
$$

Las condiciones iniciales las suponemos dadas:

$$
\mathbf{w}(\mathbf{x})=\mathbf{w}^{0} \text { para } t=0,
$$

y en general se toman como concentraciones aleatorias alrededor del punto de equilibrio. El problema está definido desde el tiempo $t_{0}=0$ hasta el tiempo final $t_{f}=T$.

\subsection{Diferencias Finitas}

En el cómputo científico, el método de las diferencias finitas es un método para calcular de manera aproximada las soluciones a las ecuaciones diferenciales usando diferencias divididas para aproximar derivadas. Para ello, primero es necesario hacer una discretización temporal y espacial del problema, es decir, hay que plantear la ecuación aproximada en términos de las diferencias finitas, para finalmente incluir las condiciones iniciales y de frontera. Esto resulta en sistemas de ecuaciones lineales que hay que resolver en cada paso de tiempo y que nos dan el valor de la solución en cada nodo de la malla.

\subsubsection{Ecuaciones en diferencias finitas}

Para aproximar las derivada temporal de una función $w(t)$ se suelen usar esquemas de primer y segundo orden, dependiendo el orden de convergencia que uno requiera. Ejemplos de este tipo de esquemas son:

$$
\frac{d w}{d t}(\tau)=\left\{\begin{array}{l}
\frac{w(\tau+\Delta t)-w(\tau)}{\Delta t}+\mathcal{O}(\Delta t), \\
\frac{3 w(\tau+\Delta t)-4 w(\tau)+w(\tau-\Delta t)}{2 \Delta t}+\mathcal{O}\left(\Delta t^{2}\right),
\end{array}\right.
$$

\footnotetext{
${ }^{1}$ Las ideas de este capítulo proceden de los cursos [33, 34, 35].
} 
donde el símbolo $\mathcal{O}(\cdot)$ representa que el error que se comete en cada aproximación es proporcional al argumento. Suponiendo que $\Delta t$ es pequeño, puede verse entonces que un esquema de segundo orden es más preciso, pero involucra la información en tres tiempos, mientras que el esquema de primer orden sólo involucra información de dos. La conveniencia de una u otra discretización dependerá del tipo de problema y la precisión que se requiera.

En cuanto a la aproximación del operador de segunda derivada (necesaria para el operador Lapalaciano), el esquema de diferencia finitas más común es el llamado esquema de diferencias centrales. Si se tiene una función que dependa de la posición $w(x)$, entonces su segunda derivada en el punto $\chi$ puede aproximarse como:

$$
\frac{d^{2} w}{d x^{2}}(\chi)=\frac{w(\chi+h)-2 w(\chi)+w(\chi-h)}{h^{2}}+\mathcal{O}\left(h^{2}\right)
$$

\subsubsection{Discretización espacial y temporal}

Supongamos que dividimos cada lado del dominio $\Omega$ en $M-1$ subintervalos de lado $h=p /(M-1)$, de tal manera que se forme una malla de cuadros con con $M^{2}$ nodos. Sean $i$ y $j$ los índices que numeren los nodos en la dirección horizontal y vertical respectivamente. Análogamente con la discretización temporal, se divide el tiempo total $T$ en $\mathrm{N}$ subintervalos de tamaño $\Delta t=T / N$ numerados con el índice $n$. Entonces la solución $\mathbf{w}(\mathbf{x}, t)$ se puede discretizar de la siguiente manera:

$$
\mathbf{w}(x, y, t)=\tilde{\mathbf{w}}(i h, j h, n \Delta t)=\mathbf{w}_{i j}^{n}
$$

En este caso $\mathbf{w}(x, y, t)$ es la solución exacta de la ecuación diferencial, mientras que usaremos $\mathbf{w}_{i j}^{n}$ como la solución de la ecuación en diferencias finitas, es decir, la solución aproximada de $\mathbf{w}$.

Con esta notación, las ecuaciones en diferencias finitas (4.4) y (4.5) pueden reescribirse respectivamente como:

$$
\begin{aligned}
& \frac{d \mathbf{w}}{d t}(n \Delta t) \approx \begin{cases}\frac{\mathbf{w}^{n+1}-\mathbf{w}^{n}}{\Delta t}, & \text { para } n=0, \ldots, N-1 \\
\frac{3 \mathbf{w}^{n+1}-4 \mathbf{w}^{n}+\mathbf{w}^{n-1}}{2 \Delta t}, & \text { para } i=1, \ldots, M-1 .\end{cases} \\
& \frac{d^{2} \mathbf{w}}{d x^{2}}(i h, j h) \approx \frac{\mathbf{w}_{i+1, j}-2 \mathbf{w}_{i, j}+\mathbf{w}_{i-1, j}}{h^{2}}
\end{aligned}
$$


La escritura de esta última ecuación puede simplificarse usando el llamado operador de diferencias centrales definido como

$$
\delta_{x} \mathbf{w}_{i, j}=\mathbf{w}_{i+1 / 2, j}-\mathbf{w}_{i-1 / 2, j},
$$

de donde es fácil deducir con poco de álgebra que:

$$
\delta_{x}^{2} \mathbf{w}_{i, j}=\delta_{x}\left(\mathbf{w}_{i+1 / 2, j}-\mathbf{w}_{i-1 / 2, j}\right)=\mathbf{w}_{i+1, j}-2 \mathbf{w}_{i, j}+\mathbf{w}_{i-1, j} .
$$

Si análogamente se define un operador $\delta_{y}$ que opere sobre el subíndice $j$, el operado Laplaciano puede escribirse de manera compacta como:

$$
\begin{aligned}
\nabla^{2} \mathbf{w} & =\left(\frac{d^{2} \mathbf{w}}{d x^{2}}+\frac{d^{2} \mathbf{w}}{d y^{2}}\right) \\
& \approx \frac{\mathbf{w}_{i+1, j}-2 \mathbf{w}_{i, j}+\mathbf{w}_{i-1, j}}{h^{2}}+\frac{\mathbf{w}_{i, j+1}-2 \mathbf{w}_{i, j}+\mathbf{w}_{i, j-1}}{h^{2}} \\
& =\frac{1}{h^{2}}\left(\mathbf{w}_{i+1, j}+\mathbf{w}_{i, j+1}-4 \mathbf{w}_{i, j}+\mathbf{w}_{i-1, j}+\mathbf{w}_{i, j-1}\right) \\
& =\frac{1}{h^{2}}\left(\delta_{x}^{2}+\delta_{y}^{2}\right) \mathbf{w}_{i, j} \quad \text { para } i, j=2, \ldots, M-1 .
\end{aligned}
$$

\subsubsection{Condiciones de frontera}

Como puede verse de la ecuación anterior, esta discretización del operador Laplaciano es válida para los puntos interiores. Significa que dado un punto interior, el Laplaciano en ese punto puede calcularse usando la información del nodo y de 4 nodos vecinos (Ver figura 4.1). Sin embargo, los puntos en la frontera sólo tienen 3 o 2 vecinos, por lo que es preciso usar las condiciones de frontera para completar la solución en estos nodos.

\section{CONDICIONES DE FLUJO NULO}

Ahora veremos en detalle cómo hacer esto para condiciones de flujo nulo en la frontera vertical de la izquierda. La generalización a los otros 3 bordes es análoga. La condición (4.2) para dicha frontera se lee cómo:

$$
\nabla \mathbf{w} \cdot\left(\begin{array}{c}
-1 \\
0
\end{array}\right)=\overrightarrow{0} \text { para } x=0
$$



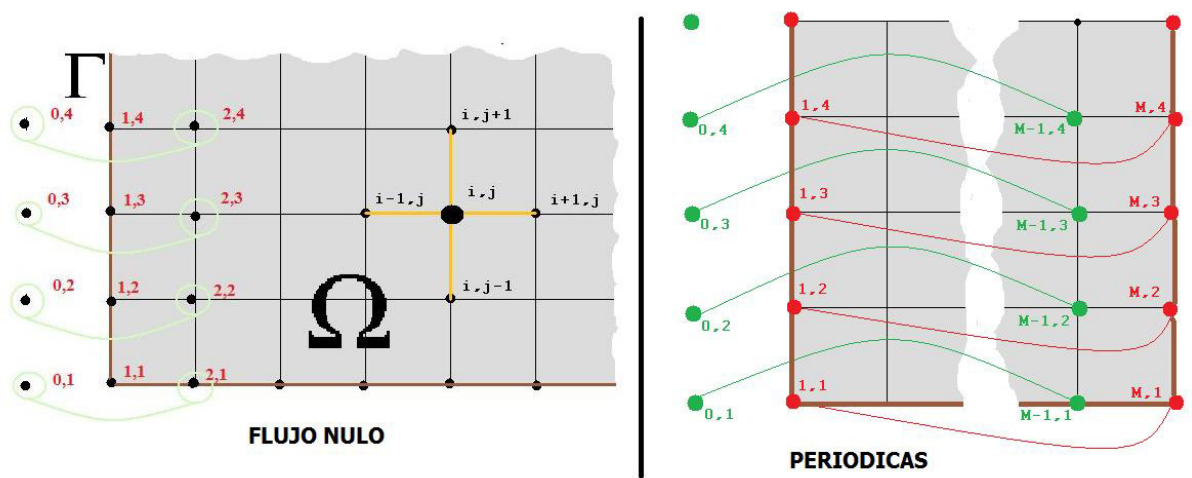

Figura 4.1: Ejemplo de discretización de un dominio $\Omega$. En el esquema de diferencias centrales, el Laplaciano en un punto se estima con cuatro vecinos. En el caso de los nodos en la frontera, la información del nodo "faltante" se deduce de las condiciones de frontera. En la figura se representan los nodos "fantasma" que permiten cumplimentar las condiciones de flujo nulo y periódicas en la frontera vertical izquierda.

Lo cual significa que $\frac{\partial \mathbf{w}}{\partial x}(x=0, y)=\overrightarrow{0}$. Si usamos una ecuación en diferencias para aproximar la derivada de la izquierda:

$$
\frac{\partial \mathbf{w}}{\partial x}(0, y)=\frac{\mathbf{w}(h, y)-\mathbf{w}(-h, y)}{2 h}+\mathcal{O}\left(h^{2}\right),
$$

uno obtiene la siguiente expresión para la solución aproximada en la frontera definida por $i=1, \forall j$ :

$$
\mathbf{w}_{0, j}=\mathbf{w}_{2, j} \quad \text { para } j=1, \ldots, M .
$$

En otras palabras, este esquema supone una columna de nodos extra (numerados por $i=0)$ tales que no pertenecen al dominio de interés $(i=$ $1, \ldots, M)$ pero que permiten seguir usando la fórmula (4.7) y a la vez cumplimentar las condiciones de frontera de flujo nulo en ese borde. Este tipo de nodos se conocen como "fantasma" y en el caso de las otras tres fronteras se puede deducir fácilmente que su valor está dado por:

$$
\begin{aligned}
\mathbf{w}_{M+1, j}=\mathbf{w}_{M-1, j} & \text { para } j=1, \ldots, M, \\
\mathbf{w}_{i, 0}=\mathbf{w}_{i, 2} & \text { para } i=1, \ldots, M, \\
\mathbf{w}_{i, M+1}=\mathbf{w}_{i, M-1} & \text { para } i=1, \ldots, M .
\end{aligned}
$$


Usando estas ecuaciones es posible extender (4.7) a todos los nodos en el entendido de que la solución está definida sólo en los nodos originales.

\section{CONDICIONES PERIÓDICAS}

En este caso, se supone que los nodos del lado derecho corresponden exactamente a los nodos del lado izquierdo, y los de la frontera de arriba corresponden a la frontera de abajo. Por ejemplo, si consideramos las fronteras verticales esto significa que:

$$
\mathbf{w}_{1 j}=\mathbf{w}_{M j} \quad \text { para todo } j .
$$

Por ello, el número de nodos horizontales donde hay que resolver el sistema es igual a $M-1$, pues el nodo $M$ es equivalente al primero. Además, los nodos fantasma que se encuentran a la izquierda de los nodos numerados por $i=1$ se corresponden con los nodos a la izquierda de los nodos numerados por $i=M$. Ver figura 4.1. Esto significa que para los nodos fantasma de la izquierda:

$$
\mathbf{w}_{0 j}=\mathbf{w}_{M-1, j} \quad \text { para todo } j .
$$

Esto se puede generalizar fácilmente para los otros tres lados del cuadrado obteniendo las siguientes condiciones para las otras fronteras:

$$
\begin{gathered}
\mathbf{w}_{1 j}=\mathbf{w}_{M j}, \\
\mathbf{w}_{0 j}=\mathbf{w}_{M-1, j}, \quad \mathbf{w}_{M+1, j}=\mathbf{w}_{2 j} \quad \text { para } j=1, \ldots, M-1, \\
\mathbf{w}_{i 1}=\mathbf{w}_{i M}, \\
\mathbf{w}_{i 0}=\mathbf{w}_{i, M-1}, \quad \mathbf{w}_{i, M+1}=\mathbf{w}_{2 j} \quad \text { para } i=1, \ldots, M-1 .
\end{gathered}
$$

\subsubsection{Esquemas de diferencias finitas para la ecuación RD}

Existen varias formas de discretizar la ecuación original (4.1) dependiendo de el orden del esquema temporal (ya sea de primer o segundo orden) y del tiempo en que se evalúe el operado Laplaciano (esquema backward o forward). Las combinaciones que usamos para esta tesis son las siguientes: 


\section{MÉTOdo Explícito (EXP)}

En el siguiente esquema totalmente explícito, la derivada temporal se evalúa con la aproximación a primer orden, mientras que los términos de la derecha de (4.1) se evalúan al tiempo $n$, es decir:

$$
\frac{\mathbf{w}_{i j}^{n+1}-\mathbf{w}_{i j}^{n}}{\Delta t}=\mathbf{R}\left(\mathbf{w}_{i j}^{n}\right)+\frac{\mathbb{D}}{h^{2}}\left(\delta_{x}^{2}+\delta_{y}^{2}\right) \mathbf{w}_{i j}^{n},
$$

o equivalentemente mediante algo de álgebra:

$$
\mathbf{w}_{i j}^{n+1}=\mathbf{w}_{i j}^{n}+\Delta t \mathbf{R}\left(\mathbf{w}_{i j}^{n}\right)+\mu\left(\delta_{x}^{2}+\delta_{y}^{2}\right) \mathbf{w}_{i j}^{n},
$$

con $n=1,2, \ldots, N$ e $i, j=1,2, \ldots, M$, con $\mu$ la matriz definida por $\mu=$ $\left(\frac{\Delta t}{h^{2}}\right) \mathbb{D}$. Por lo que para encontrar la solución al tiempo $n=1$ simplemente se sustituyen los valores de $\mathbf{w}$ y de $\mathbf{R}$ al tiempo cero obtenidos de la condición inicial $\mathbf{w}_{i j}^{0}$ definida en (4.3). Análogamente, para obtener la solución al tiempo $n+1$ sólo es necesario sustituir la información del sistema en el tiempo anterior $n$ de manera iterativa.

Este método tiene la ventaja de ser sumamente simple, sin embargo, puede demostrarse que su estabilidad está limitada, i.e. que el paso de tiempo $\Delta t$ que uno debe elegir debe ser del orden de $h^{2}$ para que el método sea estable (Ver Apéndice A y [33]). Esto significa que una solución detallada (i.e una malla muy fina) requiere de pasos de tiempo más pequeños, y por ende, de más iteraciones.

\section{Método Semi-Implícito De PRIMER orden (1-SBDF)}

Para evitar la anterior limitación, se suelen usar esquemas implícitos o semiimplícitos. Estos tienen la ventaja de que no sufren de restricciones tan severas en la elección del paso del tiempo para mantener la estabilidad, pero el costo a pagar es que, en vez de hacer una simple sustitución como en el caso explícito, ahora se tiene que resolver un sistema de ecuaciones lineales en cada iteración.

Para el esquema $1-\mathrm{SBDF}^{2}$, se usa la misma derivada temporal a primer orden, pero la discretización del Laplaciano se evalúa no al tiempo $n$ como antes, sino al tiempo $n+1$, mientras que los términos de reacción se evalúan en el tiempo $n$, obteniendo:

\footnotetext{
${ }^{2}$ Estas nombre procede de sus siglas en ingles Semi-implicit Backward Differentiation Formula, donde el 1 indica que es de primer orden en el tiempo.
} 


$$
\frac{\mathbf{w}_{i j}^{n+1}-\mathbf{w}_{i j}^{n}}{\Delta t}=\mathbf{R}\left(\mathbf{w}_{i j}^{n}\right)+\frac{\mathbb{D}}{h^{2}}\left(\delta_{x}^{2}+\delta_{y}^{2}\right) \mathbf{w}_{i j}^{n+1},
$$

por lo que al sustituir los operadores de diferencias centrales y con algo de álgebra uno puede obtener:

$$
-\mu \mathbf{w}_{i, j-1}^{n+1}-\mu \mathbf{w}_{i-1, j}^{n+1}+(1+4 \mu) \mathbf{w}_{i j}^{n+1}-\mu \mathbf{w}_{i, j+1}^{n+1}-\mu \mathbf{w}_{i, j+1}^{n+1}=\Delta t \mathbf{R}\left(\mathbf{w}_{i j}^{n}\right)+\mathbf{w}_{i j}^{n},
$$

para los nodos $i, j \in\{1, \ldots, M\}$. Haciendo la transformación de índices $k(i, j)=(M-1) i+j$ uno obtiene:

$$
-\mu \mathbf{w}_{k-1}^{n+1}-\mu \mathbf{w}_{k-M+1}^{n+1}+(1+4 \mu) \mathbf{w}_{k}^{n+1}-\mu \mathbf{w}_{k+M-1}^{n+1}-\mu \mathbf{w}_{k+1}^{n+1}=\Delta t \mathbf{R}\left(\mathbf{w}_{k}^{n}\right)+\mathbf{w}_{k}^{n},
$$

lo cual constituye para cada componente del vector $\mathbf{w}$ un sistema de ecuaciones lineales de la forma $\mathbb{A} w_{l}=\vec{b}$ donde $\mathbb{A}$ es una matriz esencialmente pentadiagonal $^{3}$ tipo sparse y $\vec{b}$ es un vector con la información del sistema al tiempo anterior. Al resolver este sistema de ecuaciones y aplicar la transformación inversa para regresar a los índices $i, j$ se encuentra la solución al tiempo $n+1$.

\section{SEMi-Implícito De SEgundo orden (2-SBDF)}

Es una variación del método anterior, solamente que ahora la aproximación temporal es de segundo orden; además se usa información de la reacción a dos tiempos distintos por medio de una extrapolación. El esquema obtenido es:

$$
\frac{3 \mathbf{w}_{i j}^{n+1}-4 \mathbf{w}_{i j}^{n}+\mathbf{w}_{i j}^{n-1}}{2 \Delta t}=\left(2 \mathbf{R}\left(\mathbf{w}_{i j}^{n}\right)-\mathbf{R}\left(\mathbf{w}_{i j}^{n-1}\right)\right)+\frac{\mathbb{D}}{h^{2}}\left(\delta_{x}^{2}+\delta_{y}^{2}\right) \mathbf{w}_{i j}^{n+1},
$$

lo cual lleva a un sistema de ecuaciones cuya estructura es similar al obtenido en el caso del esquema de primer orden, pero ahora con la siguiente forma:

$$
\begin{array}{r}
-2 \mu \mathbf{w}_{k-1}^{n+1}-2 \mu \mathbf{w}_{k-M+1}^{n+1}+(3+4 \mu) \mathbf{w}_{k}^{n+1}-2 \mu \mathbf{w}_{k+M-1}^{n+1}-2 \mu \mathbf{w}_{k+1}^{n+1}= \\
2 \Delta t\left(2 \mathbf{R}\left(\mathbf{w}_{k}^{n}\right)-\mathbf{R}\left(\mathbf{w}_{k}^{n-1}\right)+4 \mathbf{w}_{k}^{n}-\mathbf{w}_{k}^{n-1},\right.
\end{array}
$$

\footnotetext{
${ }^{3} \mathrm{La}$ estructura de la matriz se ve un poco modificada por las condiciones de frontera que se utilicen.
} 
con $\mu$ y $k$ definidos como antes. Este esquema permite utilizar un paso de discretización en el tiempo un poco mayor al esquema de primer orden.

MÉtodo implícito De DiReCCiones ALternantes (ADI ${ }^{4}$ )

Esta variación de método implícito propone dividir el intervalo de tiempo $\Delta t$ en dos partes. En la primera se toma únicamente la discretización de la segunda derivada en $x$, mientras que en la segunda parte se toma sólo la discretización de la segunda derivada en $y$. El esquema se resume en los siguientes dos pasos:

$$
\begin{gathered}
\frac{\mathbf{w}_{i j}^{n+1 / 2}-\mathbf{w}_{i j}^{n}}{\Delta t / 2}=\mathbf{R}\left(\mathbf{w}_{i j}^{n}\right)+\frac{\mathbb{D}}{h^{2}} \delta_{x}^{2} \mathbf{w}_{i j}^{n+1 / 2}, \\
\frac{\mathbf{w}_{i j}^{n+1}-\mathbf{w}_{i j}^{n+1 / 2}}{\Delta t / 2}=\mathbf{R}\left(\mathbf{w}_{i j}^{n+1 / 2}\right)+\frac{\mathbb{D}}{h^{2}} \delta_{y}^{2} \mathbf{w}_{i j}^{n+1} .
\end{gathered}
$$

Al simplificar y usar la misma transformación de índices anterior, se obtienen los siguientes sistemas de ecuaciones lineales para cada una de las dos componentes de $\mathbf{w}$ :

$$
\begin{aligned}
& -\frac{\mu}{2} \mathbf{w}_{k-M+1}^{n+1 / 2}+(1+\mu) \mathbf{w}_{k}^{n+1 / 2}-\frac{\mu}{2} \mathbf{w}_{k+M-1}^{n+1 / 2}=\frac{\Delta t}{2} \mathbf{R}\left(\mathbf{w}_{k}^{n}\right)+\mathbf{w}_{k}^{n}, \\
& -\frac{\mu}{2} \mathbf{w}_{k-1}^{n+1}+(1+\mu) \mathbf{w}_{k}^{n+1}-\frac{\mu}{2} \mathbf{w}_{k+1}^{n+1}=\frac{\Delta t}{2} \mathbf{R}\left(\mathbf{w}_{k}^{n+1 / 2}\right)+\mathbf{w}_{k}^{n+1 / 2} .
\end{aligned}
$$

Esto quiere decir que en el primer paso se resuelve un sistema para obtener la solución al tiempo intermedio $n+1 / 2$, solución que a su vez se utiliza para generar la solución al tiempo $n+1$. En cada subpaso se resuelve un sistema donde la matriz es tridiagonal y el vector de la derecha depende de la información al tiempo anterior. Este esquema tiene la ventaja de que ahora las matrices son tridiagonales, lo cual permite resolver el sistema eficientemente

\footnotetext{
${ }^{4}$ En realidad, este esquema debería llamarse semi-implícito de direcciones alternantes, pues la reacción $\mathbf{R}$ se evalúa al timpo $n$, y no al $n+1$ como requiere un esquema totalmente implícito. Este esquema requeriría resolver un sistema de ecuaciones no lineales mediante algún método iterativo. Sin embargo, nosotros usaremos sólo el método semi-implícito y seguiremos usando el nombre tradicional de método bajo este entendido.
} 
con el algoritmo de Thomas[33]. Además, en el caso de una malla cuadrada regular, el tamaño de cada uno de los sistemas tridiagonales es igual al número de nodos por lado $M$, mientras que el sistema pentadiagonal de los métodos semi-implícitos es de tamaño $M^{2}$.

\subsection{Elemento Finito}

El procedimiento que seguiremos en este trabajo es discretizar las derivadas temporales por medio de diferencias finitas, de tal manera que en cada paso del tiempo se tiene que resolver una ecuación diferencial parcial en las derivadas espaciales, utilizando para esto último el método de elemento finito.

En el método del elemento finito primero se transforma la ecuación diferencial parcial en una ecuación integral, mediante lo que se denomina la formulación variacional del problema (ó formulación débil). Luego se divide el dominio espacial en subdominios o elementos, que en nuestro caso serán triángulos. Posteriormente se aproximan las funciones en sus variables espaciales en cada elemento mediante polinomios (lineales, en nuestro caso). El resultado es un sistema de ecuaciones algebraico que se debe resolver en cada paso del tiempo. La ventaja del método de elemento finito, al discretizar sobre cada elemento, es que la malla no tiene porque ser uniforme o estructurada, haciéndolo más apropiado para dominios generales y complejos. Además, en el método de elemento finito las condiciones de frontera del tipo Neumann se adaptan de manera natural en la formulación variacional, por lo que no es necesario hacer artificios especiales como los que hicimos al aplicar el método de diferencias finitas.

Por último, queremos mencionar que también es posible dejar sin discretizar las derivadas en el tiempo y sólo discretizar las variables espaciales por medio de elemento finito. En este caso el resultado es un sistema de ecuaciones diferenciales ordinarias de primero orden en el tiempo, las cuales se pueden resolver utilizando algún método estándar como: el método de Euler, los métodos de Runge-Kutta, ó los metodos de Adams-Bashforth, entre otros. En este trabajo no seguiremos esta metodología y, por simplicidad, llevaremos a cabo la metodología señalada en el primer párrafo. 


\subsubsection{Discretización temporal}

Nuestro sistema de ecuaciones para ambas concentraciones es de la forma (4.1), con condiciones de flujo nulo en la frontera y condiciones iniciales dadas. Para ejemplificar el método de elemento finito nos concentraremos en una de las ecuaciones de (3.2), por ejemplo, aquella para $u$, dando por sentado que $v$ cumple una ecuación diferencial muy parecida, por lo que sólo restaría acoplarlas. Por ello, considérese la ecuación de reacción-difusión:

$$
\frac{\partial u}{\partial t}=D_{u} \nabla^{2} u+f(u, v)
$$

Si al igual que antes consideramos una aproximación temporal de la forma $u^{n}(\mathbf{x}) \approx u(\mathbf{x}, n \cdot \Delta t)$ con $n=0,1,2, \ldots, N$, entonces uno puede discretizar la parte temporal de manera semi-implícita como antes y obtener el siguiente problema:

$$
\begin{gathered}
\frac{u^{n+1}-u^{n}}{\Delta t}=D_{u} \nabla^{2} u^{n+1}+f^{n}(u, v), \\
\frac{\partial u}{\partial n}=0 ; \quad \text { sobre } \Omega, \mathrm{y} \\
u^{0}=u_{0} .
\end{gathered}
$$

donde $f^{n}(u, v)$ significa la función $f$ con $u$ y $v$ evaluadas al tiempo anterior $n$. Haciendo un poco de álgebra, esto significa que si tenemos la solución al tiempo $n$, podemos obtener la solución al tiempo $n+1$ resolviendo el siguiente problema:

$$
u-\nu \nabla^{2} u=\bar{f}
$$

con condiciones de frontera

$$
\nu \frac{\partial u}{\partial n}=0
$$

con $\nu=D_{u} \Delta t$ y condiciones iniciales dadas $u^{0}=u_{0}(\mathbf{x})$. En este caso hemos obviado el superíndice $n+1$, sobrentendiendo que la solución a este problema será $u^{n+1}$, la cual dependerá de la solución al tiempo anterior contenida en el término fuente de la derecha:

$$
\bar{f}=\Delta t f^{n}(u, v)+u^{n}
$$


Por ende, al discretizar el tiempo hemos transformado del problema de reacción-difusión original en una sucesión de problemas elípticos similares que se resuelven iterativamente en el tiempo. A continuación explicaremos como se resuelven los problemas elípticos del tipo (4.9)-(4.10) con elemento finito.

\subsubsection{Formulación variacional}

Si multiplicamos la ecuación (4.9) por una función de prueba $\psi(\mathbf{x}, t)$ e integramos en el dominio $\Omega$ se obtiene:

$$
\int_{\Omega} u \psi d \Omega-\nu \int_{\Omega} \psi \nabla^{2} u d \Omega=\int_{\Omega} \bar{f} \psi d \Omega
$$

Utilizando la identidad de Green, podemos hacer el cambio $\psi \nabla^{2} u=\nabla$. $(\psi \nabla u)-\nabla u \cdot \nabla \psi$ en la segunda integral, resultando en

$$
\int_{\Omega} u \psi d \Omega-\nu \int_{\Omega} \nabla \cdot(\psi \nabla u) d \Omega+\nu \int_{\Omega} \nabla u \cdot \nabla \psi d \Omega=\int_{\Omega} \bar{f} \psi d \Omega
$$

Para la segunda integral podemos usar el teorema de Gauss:

$$
\int_{\Omega} \nabla \cdot(\psi \nabla u) d \Omega=\int_{\Gamma} \psi(\nabla u \cdot \hat{n}) d \Gamma
$$

siendo $\hat{n}$ el normal unitario. Sin embargo, de la condición de frontera(4.10), esta integral se anula ${ }^{5}$, por lo que la ecuación integral resultante es:

$$
\int_{\Omega} u \psi d \Omega+\nu \int_{\Omega} \nabla u \cdot \nabla \psi d \Omega=\int_{\Omega} \bar{f} \psi d \Omega
$$

Para que lo anterior tenga sentido basta que las funciones de prueba $\psi \mathrm{y}$ la solución $u$ y sus derivadas sean cuadrado integrables en el sentido de las distribuciones generalizadas. En otras palabras se requirió que $u$ y $\psi$ fueran

\footnotetext{
${ }^{5}$ Debe notarse que esta integral de frontera también se anula para cuando deseamos resolver la misma ecuación con condiciones periódicas. En este caso se debe a que no existe frontera del dominio. Los nodos que cubren el lado derecho y superior equivalen a los nodos izquierdo e inferior respectivamente. Por ello al "cruzar" el borde derecho es como si se entrara por el lado izquierdo formando un continuo. En elemento finito esto se logra numerando estos $2 M$ nodos extra de arriba y derecha con la misma etiqueta que los nodos de abajo e izquierda respectivamente. Al igual que en el caso de diferencias finitas, esto significa que los nodos de relevancia son solamente los $(M-1)^{2}$ nodos restantes.
} 

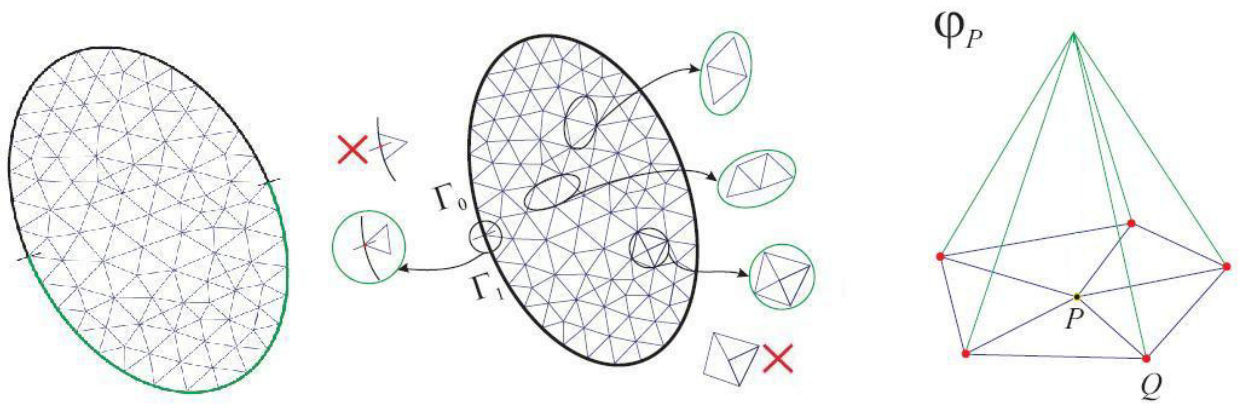

Figura 4.2: De izquierda a derecha: Elementos de una triangulación del dominio; requerimientos de la triangulación, y finalmente, ilustración de una función base piramidal. La función base piramidal vale cero en todos los otros nodos de la malla, no mostrados en el dibujo.

funciones del espacio de Hilbert $H^{1}(\Omega)$. Como se tomo $\psi$ función $H^{1}(\Omega)$ arbitraria, el problema variacional consiste en encontrar la función $u$ que cumpla con la ecuación (4.12) para todo $\psi \in H^{1}(\Omega)$

\subsubsection{Funciones base y proyección en elementos}

El método de elemento finito requiere la subdivisión del dominio $\Omega$ en un conjunto de elementos más pequeños, siendo los triángulos los más utilizados. Las características de la triangulación $\tau_{h}$ del dominio $\Omega$ son: a) La unión de todos los triángulos forma el dominio $\Omega$ y su frontera; y b) Dos triángulos de la subdivisión o bien no se intersectan, o cuando se intersectan lo hacen en una arista ó vértice común. Ver Figura 4.2 .Sea $\Sigma_{h}$ el conjunto de todos los vértices $P=\left(x_{i}, y_{i}\right)$ de la triangulación. El subíndice $h$ puede verse como un parámetro que depende de la discretización ${ }^{6}$, por lo que denotaremos como $u_{h}$ a la solución aproximada del problema variacional.

El problema aproximado se obtiene escribiendo la solución aproximada como combinación lineal de funciones base $\phi_{P}(\mathbf{x})$ de la forma:

$$
u_{h}(\mathbf{x})=\sum_{P \in \Sigma_{h}} \alpha_{P} \phi_{P}(\mathbf{x}),
$$

Eligiendo la funciones base $\phi_{P}$ como funciones continuas que son lineales en

\footnotetext{
${ }^{6}$ El sentido matemático del parámetro $h$ podrá verse más claramente en el Apéndice C.
} 
cada triángulo, se obtiene funciones cuya forma es una pirámide centrada en el nodo, de tal modo que su valor es 1 en el vértice central $P$ y 0 en todos los vértices restantes de la maya $Q=\left(x_{j}, y_{j}\right)$. Es posible demostrar que los coeficientes $\alpha_{P}$ coinciden con el valor de la función en ese punto $u_{h}(P)$. De tal manera que se tiene:

$$
\begin{gathered}
u_{h}(\mathbf{x})=\sum_{P \in \Sigma_{h}} u_{h}(P) \phi_{P}(\mathbf{x}) \\
\psi_{h}(\mathbf{x})=\phi_{Q}(\mathbf{x}) .
\end{gathered}
$$

con $Q$ corriendo sobre todos los nodos de la malla. Sustituyendo estos aproximaciones en la ecuación (4.12), se tiene:

$$
\sum_{P \in \Sigma_{h}} u_{h}(P)\left(\int_{\Omega} \phi_{P} \phi_{Q} d \Omega+\nu \int_{\Omega} \nabla \phi_{P} \cdot \nabla \phi_{Q} d \Omega\right)=\int_{\Omega} \bar{f} \phi_{Q} d \Omega
$$

para cada uno de los $M_{t}$ vértices de la triangulación . Esto puede verse como un sistema de ecuaciones lineales de tamaño $M_{t}$ de la forma:

$$
\mathbb{A}_{h} \mathbf{U}_{h}=(\mathbb{M}+\nu \mathbb{K}) \mathbf{U}_{h}=\vec{b}_{h},
$$

siendo la matriz $\mathbb{A}$ la suma de la matriz de masa $\mathbb{M}$ más $\nu$ veces la matriz de rigidez $\mathbb{K}$, cuyos respectivos coeficientes son:

$$
\begin{gathered}
\mathbb{M}_{i j}=\int_{\Omega} \phi_{i} \phi_{j} d \Omega, \\
\mathbb{K}_{i j}=\int_{\Omega} \nabla \phi_{i} \cdot \nabla \phi_{j} d \Omega .
\end{gathered}
$$

El sistema lo completa el vector de carga $\vec{b}$ cuyos coeficientes son:

$$
b_{j}=\int_{\Omega} \bar{f} \phi_{j} d \Omega
$$

y el vector solución $\{U\}_{i}=\left\{u_{h}\left(x_{i}, y_{i}\right)\right\}_{i}$. Así, la solución al problema aproximado se reduce a resolver un sistema lineal de ecuaciones de tamaño $M_{t}^{2}$. Esto quiere decir que conforme más fina sea la triangulación, mayor será el tamaño del sistema lineal. Basta decir que la convergencia de la aproximación 4.13 a la solución del problema variacional 4.12 está garantizada por el 
teorema de Lax-Milgram generalizado, así como la convergencia a la solución exacta cuando la discretización es muy fina. Los detalles de este teorema se estudiarán en el Apéndice C.

El cálculo explícito de las funciones base y de las distintas integrales involucradas en el problema anterior se facilita en gran manera por la utilización del llamado elemento maestro. Esencialmente, este cálculo requiere: 1) hacer una transformación afín de cada triángulo en un elemento de referencia donde es más sencillo hacer los cálculos y 2) separar las diversas contribuciones de las integrales en todo el dominio $\Omega$ en la suma de las contribuciones por cada elemento. Los detalles de este proceso no se incluyen en esta tesis, para lo cual se remite el lector a cualquier libro que incluya aspectos prácticos del método de elemento Finito, por ejemplo [36].

\section{Algunas notas sobre la programación.}

1) Todos los programas se realizaron en Matlab, que pueden encontrarse junto con sus resultados en un archivo adjunto a este documento escrito. Los programas pueden encontrarse en el Apéndice D.

2) Respecto al acoplamiento entre $u$ y $v$, tanto para diferencias finitas y elemento finito: en ambos casos, una vez obtenida una solución para $u$ al tiempo $n$, esta información se actualiza en la reacción de $v$ para obtener una mejor aproximación de $v^{n}$. Con estas dos soluciones se obtiene $u^{n+1}$, que se actualiza para la reacción de $v^{n+1}$ y así sucesivamente.

3) En ambos métodos, los esquemas resultan en sistemas de ecuaciones lineales de la forma $\mathbb{A} w=\vec{b}$, donde $\mathbb{A}$ es una matriz simétirca y positiva definida del tipo sparse. Aprovechando la programación en Matlab utilizamos los comandos $>>\operatorname{sparse}(A)$ y $>>w=A \backslash b$ para una eficiente resolución y almacenamiento de los sistemas de ecuaciones.

4) El método ADI resulta en sistemas tridiagonales que se pueden resolver por medio del algoritmo de Thomas solamente para el caso de condiciones de frontera de flujo nulo. En el caso de condiciones periódicas no es posible hacer esta reducción por lo que tiene que resolverse el sistema completo.

5) En el caso de mallas no estructuradas para dominio irregulares, su generación se hizo con el programa GID [37]. No obstante para la programación y las gráficas se utilizó igualmente Matlab.

6) Respecto al criterio de convergencia. En el caso en que los resultados se reproducen de otro autor, usualmente está indicado tiempo $T$ que dura 
el mecanismo en alcanzar su estabilidad. Cuando este dato no esté indicado, obedecimos únicamente al criterio de semejanza con los resultados publicados. Análogamente respecto al error numérico, supusimos que el autor ya realizó las pruebas de refinamiento temporal y de la malla y utilizamos el intervalo de tiempo $\Delta t$ y el tamaño de malla $h$ que él indica. Cuando estos datos no aparecen, se buscan mediante prueba y error hasta alcanzar la similitud de resultados.

7) Las pruebas de error que consiste en refinar la malla y/o el intervalo de tiempo sólo se hicieron para los parámetros generados por nosotros. Igualmente, para nuestros resultados se utilizó un criterio de paro basado en la norma del vector solución, el cual indica que si en un una iteración la diferencia de la norma del vector al tiempo $n+1$ y al tiempo $n$ es menor a $\epsilon \sim 1 \times 10^{-8}$, se ha alcanzado la estabilidad.

8) Para el caso de elemento finito, se utilizaron solamente elementos triangulares y la integración numérica se realizó mediante el método de Simpson.

9) La ejecución de los programas se realizaron en una computadora portátil HP Pavillon dv6000 con procesador AMD Turion 64 bits, 4096 MB en memoria RAM y velocidad de $667 \mathrm{MHz}$.

10) Algunas propiedades teóricas de los métodos numéricos de diferencias finitas (estabilidad, consistencia y convergencia) se bosquejan en los Apéndices A y C. 


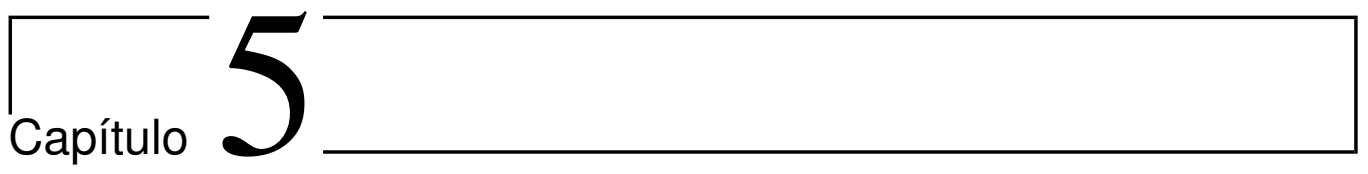

\section{Miscelánea de patrones obtenidos por simulación numérica}

En este capítulo tratamos de resumir algunas de las características más generales y relevantes de los patrones de Turing. Para ello retomamos resultados publicados con anterioridad con el objetivo no sólo de confirmar la corrección de nuestros programas, sino también para estudiar la relación que podrían tener con algún sistema concreto.

1) De la referencia [34]. Se usa la reacción de Schnakenberg (3.19):

$$
\begin{aligned}
& u_{t}=\gamma\left(a-u+u^{2} v\right)+\nabla^{2} u \\
& v_{t}=\gamma\left(b-u^{2} v\right)+d \nabla^{2} v
\end{aligned}
$$

en el dominio unidimensional $[0,1]$ con condiciones de frontera periódicas y condiciones iniciales que van como oscilaciones alrededor de las concentraciones de equilibrio:

$$
\begin{aligned}
& u(x, 0)=u_{0}+0.001 \sum_{j=1}^{25} \frac{\cos (2 \pi j x)}{j}, \\
& v(x, 0)=v_{0}+0.001 \sum_{j=1}^{25} \frac{\cos (2 \pi j x)}{j} .
\end{aligned}
$$




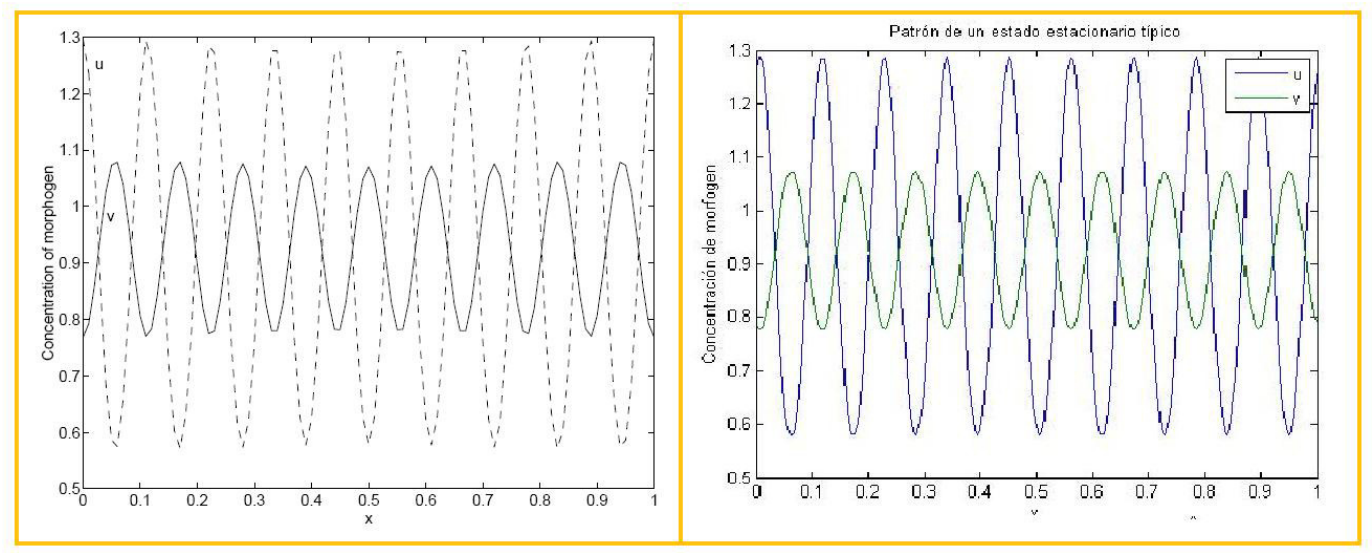

Figura 5.1: Izquierda: Patrón estacionario al tiempo T=2.5, tomado de Ruuth [34]. Los parámetros son $a=0.126779, b=0.792366, d=10, \gamma=10000, u_{0}=$ $0.919145, v_{0}=0.937903$. Derecha: Patrón reproducido con el método 2-SBDF con una malla de 200 nodos y $\Delta t=5 \times 10^{-5}$.

Este ejemplo sencillo confirma que un mecanismo RD puede dar lugar a patrones para las concentraciones $u$ y $v$. También se observa que las oscilaciones son periódicas, de amplitud constante y, en el caso particular de la reacción de Schnakenberg, los patrones de $u$ y $v$ ocurren con desfase, es decir, los mínimos de $u$ corresponden a máximos de $v$ y viceversa. Ver Figura 5.1.

2) De referencia [38] . Para la misma reacción de Schnakenberg (3.19) en el dominio [-1,1], con condiciones iniciales de ruido a aleatorio y condiciones de frontera de flujo nulo.

Se observa que, incluso partiendo de condiciones aleatorias, el mecanismo RD de Turing selecciona los modos adecuados para la formación de patrones estacionarios, partiendo únicamente de alguna perturbación espacial. Ver Figura 5.2.

3) Tomado de la referencia [38]. La misma reacción (3.19) con los mismos valores de parámetros, pero ahora para un dominio bidimensional de un cuadrado unitario. Las condiciones son de flujo nulo en la frontera y condiciones iniciales dadas por: 


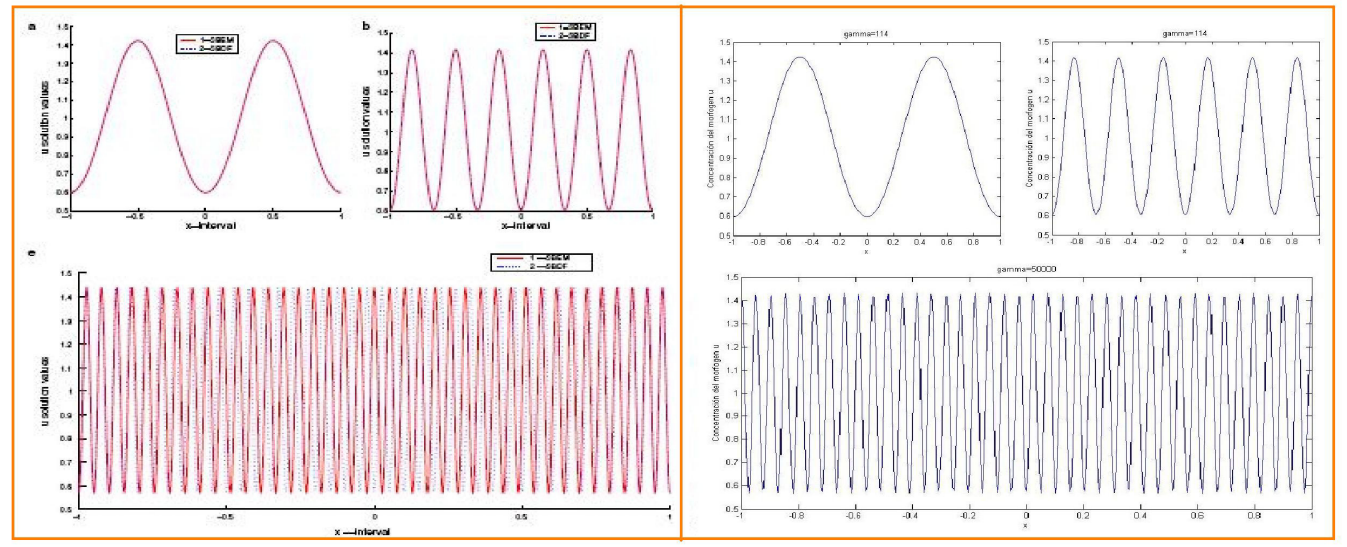

Figura 5.2: Izquierda: Patrones para distinto valores de $\gamma=114,1000,5000$ respectivamente y un tiempo $\mathrm{T}=5$, tomado de Mazdvamuse [38]. Los parámetros son: $a=0.1, b=0.9$ y $\gamma$ igual a 114,1000 y 50000 respectivamente . Derecha: Reproducción de patrones con método 1-SBDF y una malla de 400 nodos, $\Delta t=5 \times 10^{-5}$.

$$
\begin{aligned}
& u(x, y, 0)=u_{0}+0.0016 \cos (2 \pi(x+y))+0.01 \sum_{j=1}^{8} \cos (2 \pi j x), \\
& v(x, y, 0)=v_{0}+0.0016 \cos (2 \pi(x+y))+0.01 \sum_{j=1}^{8} \cos (2 \pi j x) .
\end{aligned}
$$

Los resultados se resumen en la Figura 5.3. Se observan patrones con manchas en todos los casos, salvo cuando el tamaño del dominio es comparable con el de la forma del patrón.

4) Ejemplo tomado de [39]. Se utiliza la misma reacción (3.19) para un cuadrado de lado 1.1. Las condiciones iniciales son oscilaciones mas ruido aleatorio alrededor del punto de equilibrio.

Fijando los valores de $\gamma=10000$ y $d=20$, se pueden encontrar distintos patrones variando los coeficientes $a$ y $b$. En la Figura 5.4 se observan de izquierda a derecha para la pareja $(a, b)$ : franjas $(0.07,1,61)$, zigzags $(0.14,1.34)$, hexágonos $\mathrm{H} 1(0.02,1.77)$ y hexágonos H2(0.1,1.35).

5) Tomado de [39]. La misma reacción de Schnakenberg (3.19) en un 


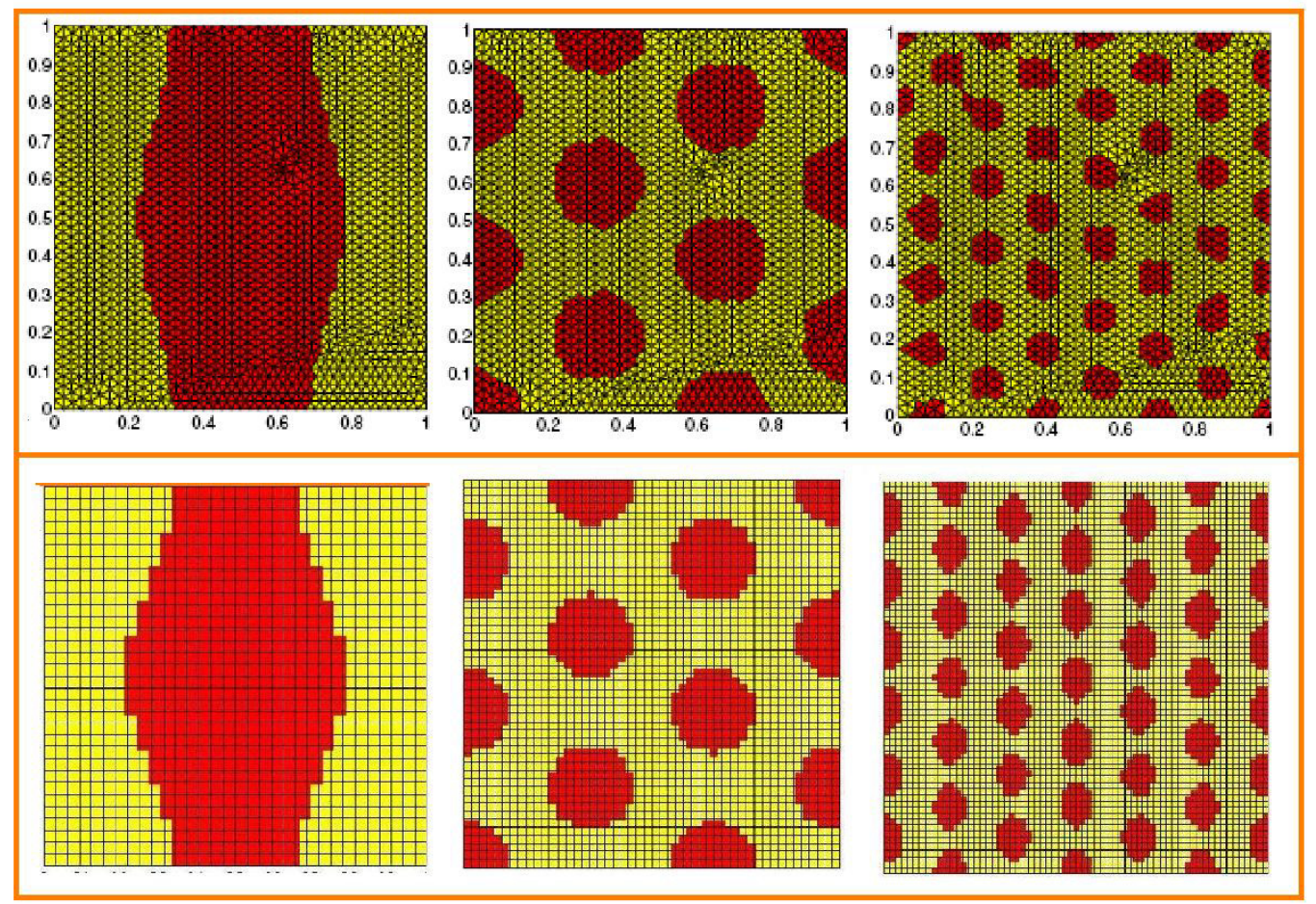

Figura 5.3: Arriba: patrones obtenidos por Madzvamuse [38], variando solo el parámetro $\gamma=114,1000,5000$ respectivamente después de un tiempo final $T=5$. Los otros parámetros son: $\mathrm{a}=0.126779, \mathrm{~b}=0.792366$ y d=10. Abajo: Reproducción utilizando distintos métodos. Utilizamos distintos métodos para cada figura: a) 2 -SBDF, $\Delta t=1 \times 10^{-4}$, malla de $32 \times 32$; b) Explícito, $\Delta t=1 \times 10^{-5}$, malla de $48 \times 48$; c) 1 -FBDF, $\Delta t=5 \times 10^{-5}$, malla de $64 \times 64$. 


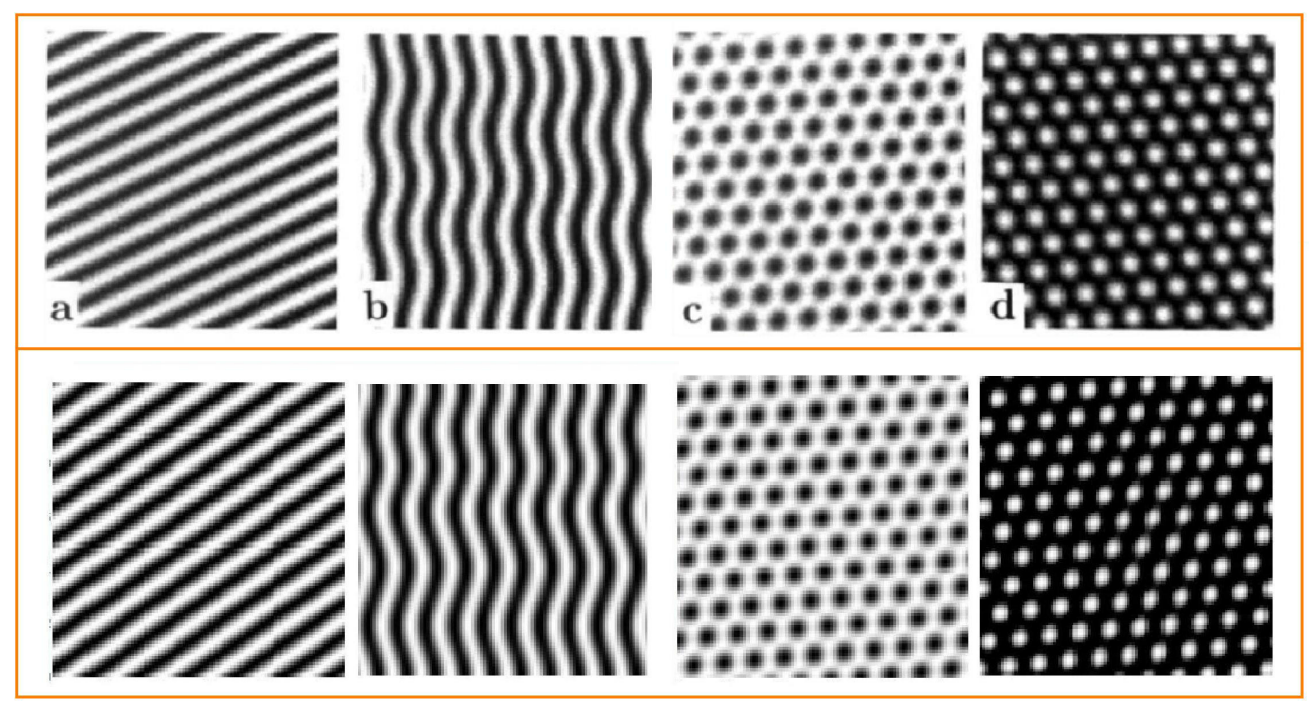

Figura 5.4: Arriba: patrones regulares para la reacción de Schnakenberg según cambios en $(a, b)$, ver texto para detalles. Tomado de Dufiet [39]. Abajo: Reproducción distintos métodos en una malla regular de $96 \times 96$. De izquierda a derecha a) 2-SBDF con $\Delta t=5 \times 10^{-5}$; b) EXP con $\Delta t=1 \times 10^{-6}$; c) 1 SBDF con $\Delta t=1 \times 10^{-5}, \mathrm{y}$ d) ADI con $\Delta t=1 \times 10^{-5}$. El tiempo final en todos los casos fue $T=1$. 


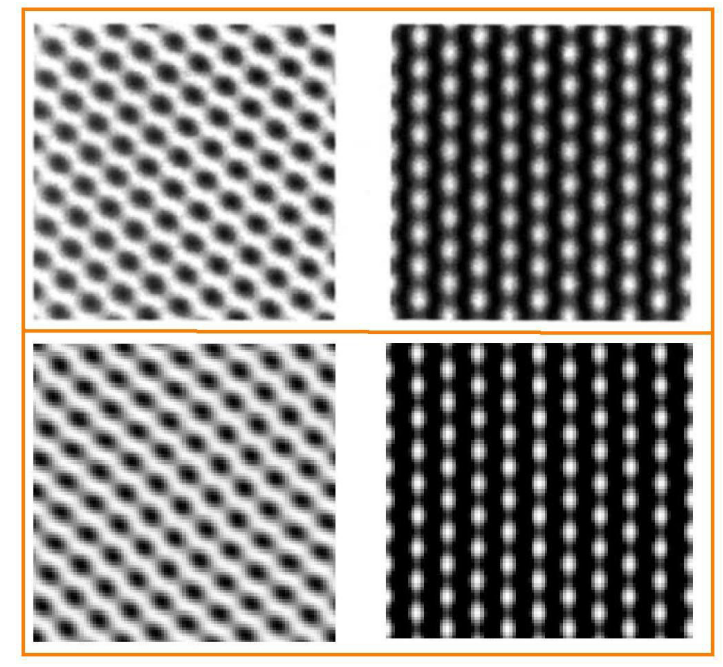

Figura 5.5: Arriba: Patrones mezclados de franjas y arreglos hexágonales obtenidos por Dufiet [39]. Los parámetros usados son $d=20$ y $\gamma=1000$ como antes y $a=1.535$ y $b=1.35$ para la transición de hexágonos a franjas, y $a=1.535, b=1.241$ para el caso inverso. Abajo: Reproducción usando método $2-\mathrm{SBDF}, \Delta t=1 \times 10^{-5}$ y una malla de $96 \times 96$.

cuadrado de lado 1.1, con condiciones periódicas a la frontera, y condiciones iniciales oscilatorias con ruido añadido. Lo que se observa es la transición de hexágonos a franjas y viceversa, debido a que los parámetros se encuantran en la frontera entra la región de uno y otro tipo de parámetros.

Se analiza el comportamiento transitorio entre un patrón hexagonal H1 que decae en franjas y viceversa. Ello puede observarse en un cuadrado de lado 1.1, con condiciones periódicas a la frontera, condiciones iniciales oscilatorias con ruido añadido. Ver Figura 5.5.

6) Tomado de [39]. La reacción (3.19) para dos dominios diferentes. En el primer caso se trata de un cuadrado de lado 2, y en el segundo caso para un rectángulo de medidas $1.99 \times 0.49$. En ambos casos se toman condiciones de flujo nulo en las fronteras y condiciones iniciales totalmente aleatorias.

Para ambos dominios se toman la misma combinación de parámetros y lo que se observa es la tendencia de las franjas a alinease paralelamente a alguna de los bordes, en el caso de condiciones de flujo nulo. Ver Figura 5.6. 

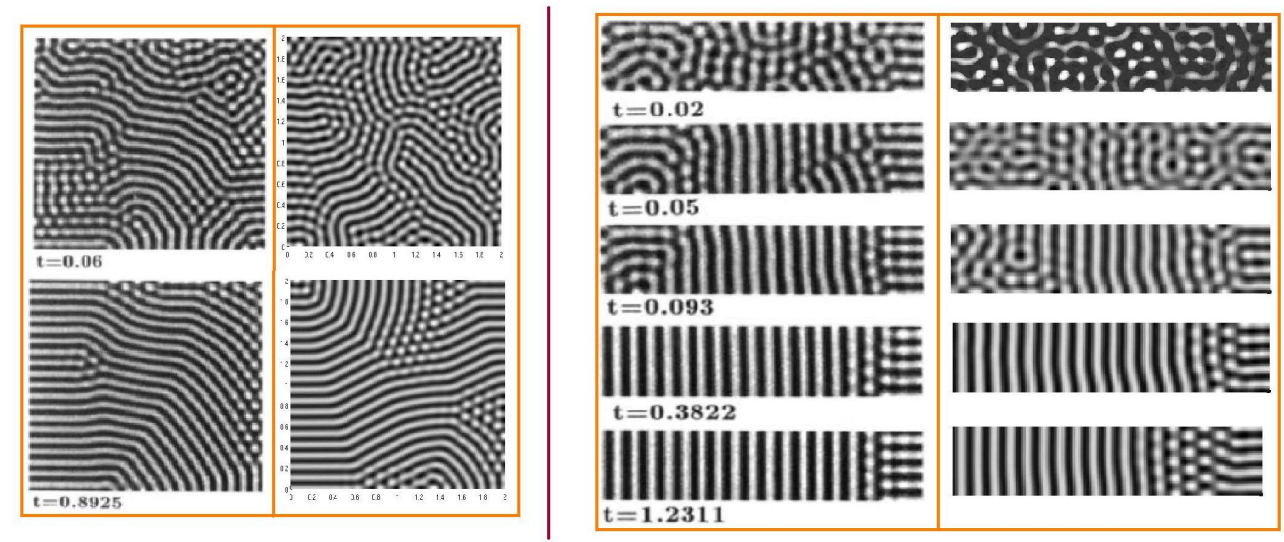

Figura 5.6: Con condiciones de flujo nulo, el patrón tiende a alinearse con los bordes. Patrones para dos dominios diferentes y el desenvolvimiento para varios tiempos . Tomado de Dufiet [39]. Los parámetros son $a=0.14, b=$ $0.35, d=20$ y $\gamma=10000$. La reproducción se hizo utilizando los métodos $2-\mathrm{SBDF}$ y ADI respectivamente. En ambos casos, se tomo $\Delta t=1 \times 10^{-5} \mathrm{y}$ $h=0.01$.

7) Los siguientes ejemplos proceden de [40]. Se utiliza la reacción de Gray Scott:

$$
\begin{aligned}
& \frac{\partial u}{\partial t}=D_{u} \nabla^{2} u-u v^{2}+F(1-u), \\
& \frac{\partial v}{\partial t}=D_{v} \nabla^{2} v-(F+k) v+u v^{2} .
\end{aligned}
$$

donde $F$ y $k$ son los parámetros de la reacción. En este caso $u$ funciona como el inhibidor y los coeficientes de difusión fijos son $D_{u}=2 \times 10^{-5}$ y $D_{v}=10^{-5}$. Se utilizan condiciones periódicas a la frontera en un dominio cuadrado de lado 2.5. Se lleva a cabo una discretización de 256 nodos en cada lado. Las condiciones iniciales proceden de la siguiente manera: Primero se fija $u=1$ y $v=0$ en todo el dominio; luego en un cuadrado ubicado al centro conformado por 20 nodos por lado se cambia $u=1 / 2$ y $v=1 / 4$ formando un hueco y un pulso respectivamente; finalmente todo el sistema se perturba con ruido aleatorio de intensidad $\pm 1 \%$.

A diferencia de los patrones reproducidos anteriormente de [39], las diversas formas (círculos y franjas) aparecen por crecimiento y autoreplicación. 


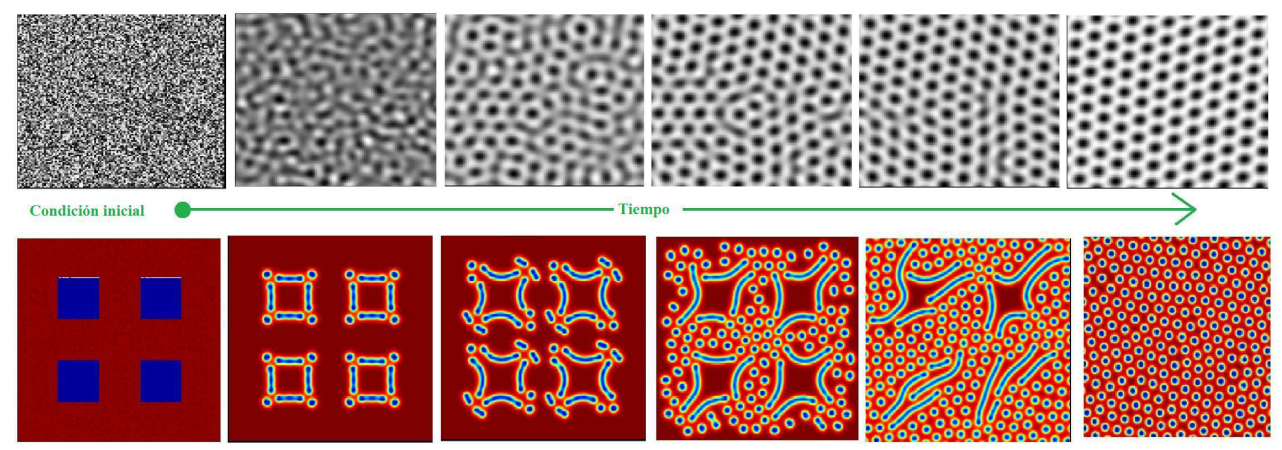

Figura 5.7: Comparación de el desarrollo de patrones en Dufiet [39] y Pearson [40]. En el primer caso se forman manchas en todo el dominio que se organizan por efectos de frontera. En el segundo caso las formas surgen por autoreplicación de círculos o por crecimiento/decrecimiento en el caso de franjas.

Todos los patrones antes obtenidos se formaban como reorganización de manchas por efectos de frontera. En el caso de las condiciones iniciales sugeridas por Pearson las manchas se dividen y duplican llenando el espacio, mientras que las franjas se elongan o rompen en círculos dependiendo el tipo de patrón. Ver Figura 5.7. Pearson encuentra hasta doce tipos distintos de patrones de los cuales nosotros sólo reprodujimos algunos. Ver Figura 5.8 .

8) Tomado de [41]. En este caso se explora el efecto del la forma del dominio en los patrones. Para ello se utiliza una reacción BVAM ${ }^{1}$ :

$$
\begin{aligned}
& \frac{\partial u}{\partial t}=D \delta \nabla^{2} u+\alpha u\left(1-r_{1} v^{2}\right)+v\left(1-r_{2} u\right), \\
& \frac{\partial v}{\partial t}=\delta \nabla^{2} u+\beta v\left(1+\frac{\alpha r_{1}}{\beta} u v\right)+u\left(r_{2} v-\alpha\right) .
\end{aligned}
$$

La forma y el tamaño exacto del dominio no se específica, por lo que nuestros resultados son sólo cualitativos. Lo único que se puede decir es que el dominio es irregular y que las condiciones de frontera son tipo Neumann: una fuente de $u$ en la curva frontera superior y flujo nulo en las otras fronteras.

\footnotetext{
${ }^{1}$ Llamada así por las siglas de sus autores: Barrio, Varea, Aragón y Maini. Cabe decir que esta misma reacción se puede escribir en su forma adimensional, la cual usaremos después.
} 


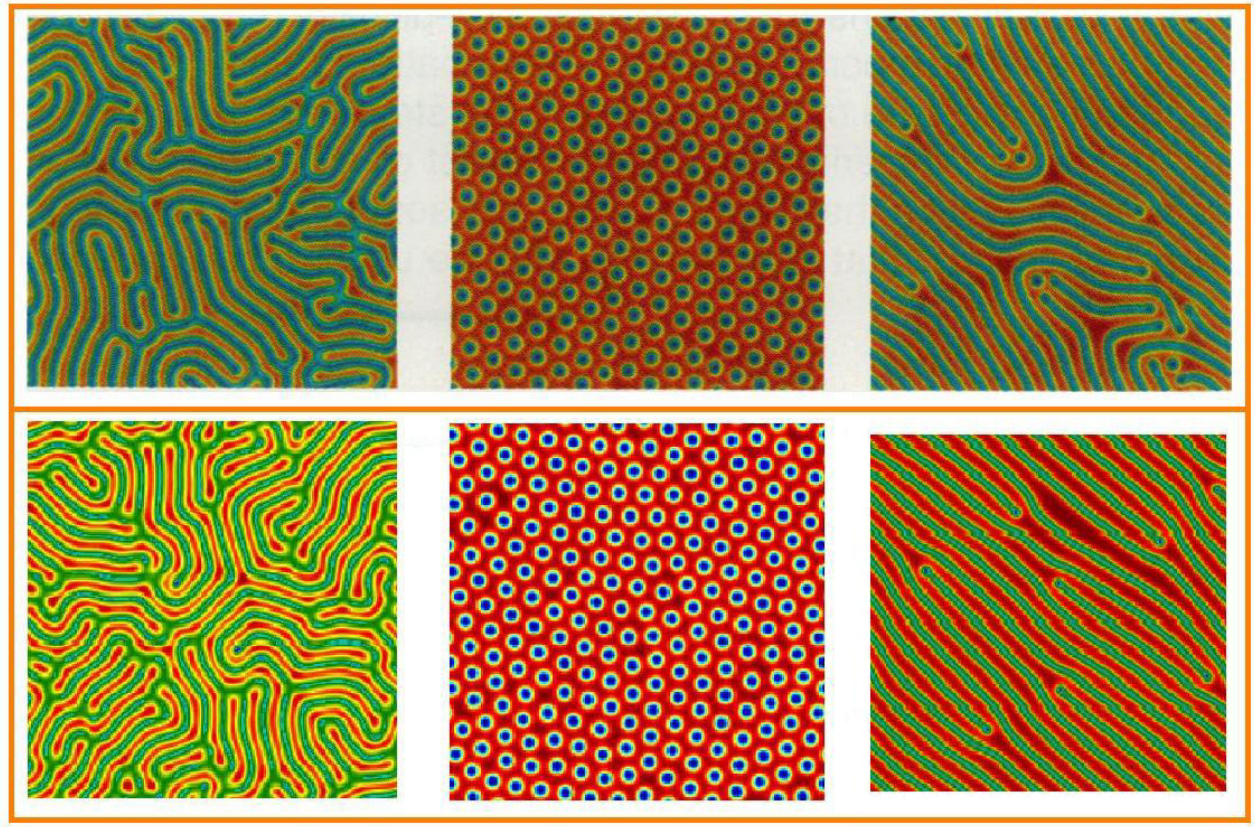

Figura 5.8: Arriba. Patrones característicos del sistema de Gray-Scott con las condiciones iniciales sugeridas por Pearson [40]. Los parámetros $F$ y $k$ determinan el tipo de patrón: el laberíntico $(F=0.05, k=0.063)$, el hexagonal $(F=0.04, k=0.065)$; y el de franjas $(F=0.04, k=0.065)$ respectivamente. Abajo : Patrones reproducidos con elemento finito. Se usa un intervalo de tiempo es de $\Delta t=5$ y el tiempo total es $\mathrm{T}=200,000$. 
Las condiciones iniciales son de ruido aleatoria alrededor de la concentración de equilibrio $\left(u_{0}, v_{0}\right)=(0,0)$.

El dominio tiene la forma de aleta de un pez y puede observarse que éste produce irregularidades y defectos en los distintos patrones. Ver Figura 5.9. En las franjas $\left(r_{1}=3.5, r_{2}=0\right)$, la forma del dominio produce cruzamientos y faltas de paralelismo; en las manchas $\left(r_{1}=0.02, r_{2}=0.2\right)$, produce un ordenamiento que no corresponde al hexagonal, y finalmente en el patrón en forma de panal $\left(r_{1}=3.5, r_{2}=0.2\right)$ se producen distorsiones en la forma de los huecos y en su orientación.

Otra observación realizada por el autor es que valores con mayor $r_{2}$ favorecen las manchas mientras que si $r_{1}$ predomina se producen franjas. Esto se debe a que $r_{1}$ y $r_{2}$ corresponden a los términos cúbicos y cuadráticos de la reacción respectivamente. Es ampliamente sabido que la interacción cúbica favorece las franjas mientras que las cuadráticas producen patrones de manchas [41].

9)El siguiente ejemplo también es de [41]. Se analiza el comportamiento de dos sistemas de Turing acoplados mediante las ecuaciones:

$$
\begin{gathered}
\frac{\partial u}{\partial t}=D \delta \nabla^{2} u+\alpha u\left(1-r_{1} v^{2}\right)+v\left(1-r_{2} u\right)+q_{1} u^{\prime}+q_{2} u^{\prime} v+q_{3} u^{\prime} v^{2} \\
\frac{\partial v}{\partial t}=\delta \nabla^{2} u+\beta v\left(1+\frac{\alpha r_{1}}{\beta} u v\right)+u\left(r_{2} v-\alpha\right)-q_{2} v^{\prime} u-q_{3} v^{2} u^{\prime} \\
\frac{\partial u^{\prime}}{\partial t}=D^{\prime} \delta^{\prime} \nabla^{2} u^{\prime}+\alpha u^{\prime}\left(1-r_{1} v^{\prime 2}\right)+v\left(1-r_{2}^{\prime} u^{\prime}\right), \\
\frac{\partial v^{\prime}}{\partial t}=\delta^{\prime} \nabla^{2} u^{\prime}+\beta^{\prime} v^{\prime}\left(1+\frac{\alpha r_{1}}{\beta^{\prime}} u^{\prime} v^{\prime}\right)+u^{\prime}\left(r_{2}^{\prime} v^{\prime}-\alpha^{\prime}\right) .
\end{gathered}
$$

Los términos $q_{1}, q_{2}, q_{3}$ determinan el orden de la interacción lineal, cuadrática y cúbica respectivamente. Se analiza el comportamiento del sistema con sólo una interacción de intensidad $q_{i}= \pm 0.55$ a la vez, donde el índice $i$ determina el orden de la interacción y el signo determina si es interacción positiva o negativa.

Lo que se observa es que el tipo de patrón obtenido cambia con el signo de la interacción. Además, en el orden lineal los dos sistemas de Turing producen patrones semejantes a los obtenidos anteriormente (hexágonos). 


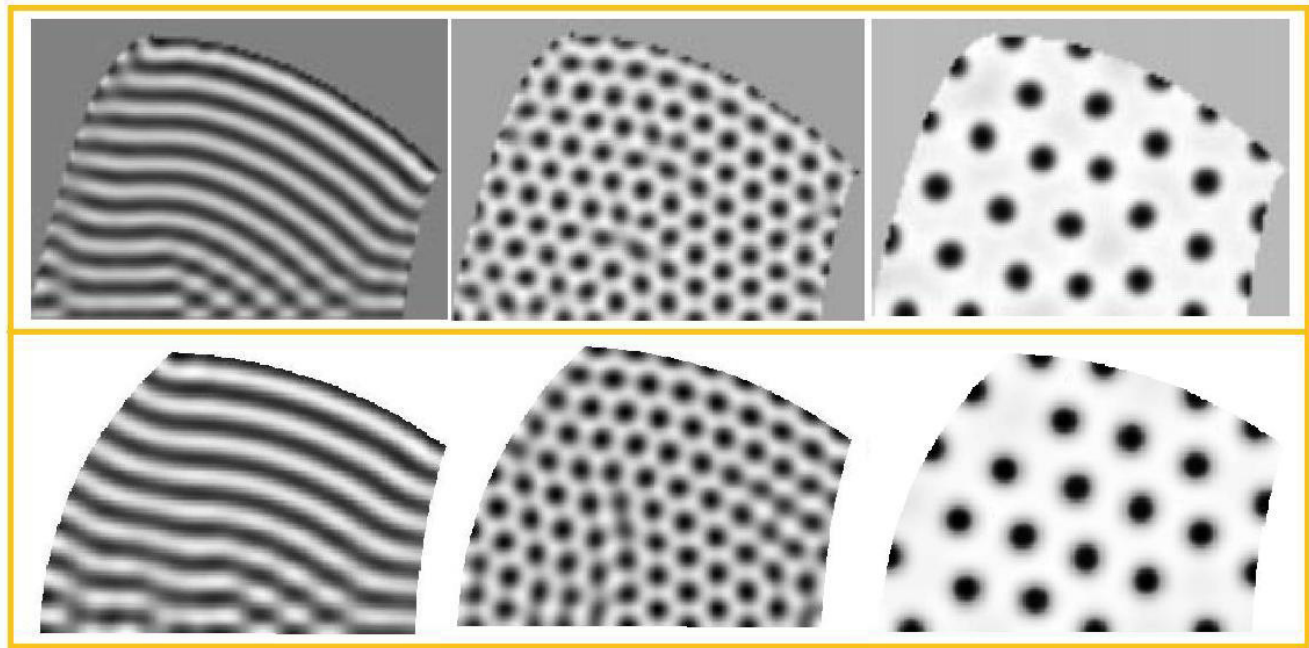

Figura 5.9: Arriba: Distintos irregularidades producida por la forma irregular del dominio en franjas, patrones hexagonales del tipo $H_{0}$ y $H_{\pi}$ encontrados por Barrio, 1999. Las primeras dos figuras utilizan los paráme$\operatorname{tros} \alpha=0.398, \beta=-0.4, \delta=2, D=0.122$. La última figura utiliza $\alpha=0.899, \beta=-0.91, \delta=2, D=0.526$. La fuente es de intensidad 0.005. Abajo: reproducciones hechas con elemento finito con un mallado no estructurado de triángulos con 100 nodos en cada lado. Se utilizó un intervalo de tiempo igual a la unidad y se hicieron 5,000 iteraciones. La escala de grises varía de -0.4 a 0.4 al igual que en el artículo. 
64 Miscelánea de patrones obtenidos por simulación numérica

Sin embargo si la interacción es cuadrática se observan patrones laberínticos o tipo mosaicos, los cuales no se obtienen con un sólo sistema de Turing. Ver Figura 5.10. 


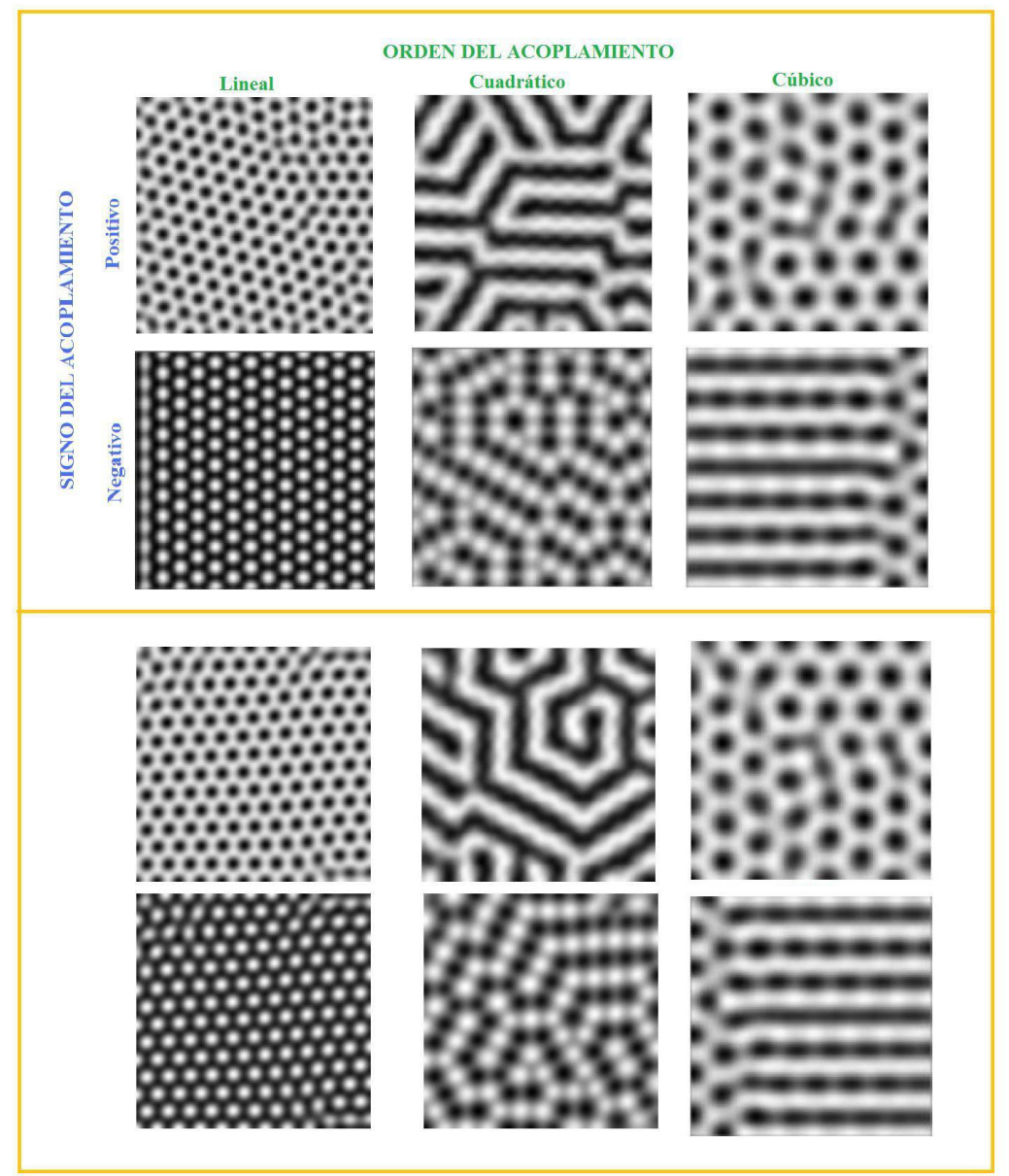

Figura 5.10: Arriba: Imagen de Barrio, 1999, analizando diversos tipos de acoplamiento entre dos sistemas de Turing. Los valores de los parámetros son $r_{1}=3.5, r_{2}=0, D=0.516, \alpha=0.899, \beta=-0.91, r_{2}^{\prime}=0.2, D^{\prime}=$ $0.122, \alpha^{\prime}=0.398, \beta^{\prime}=0.4, \delta=\delta^{\prime}=2$. Abajo: nuestra reproducción usando elemento finito con una malla estructurada de 100 nodos por lado y un paso de tiempo $\Delta t=1$. La escala de grises varía de -0.4 a 0.4 al igual que en el artículo. 
66 Miscelánea de patrones obtenidos por simulación numérica 


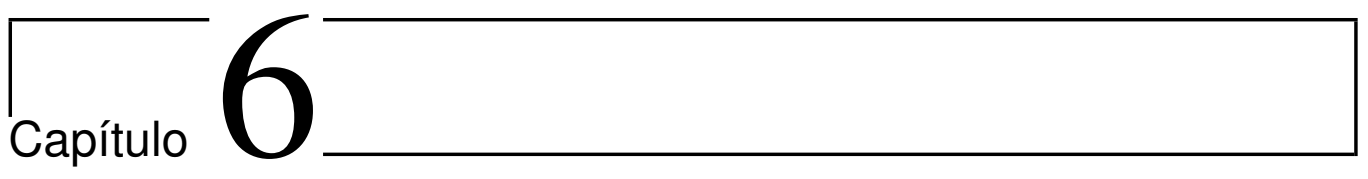

\section{Patrones en peces Pseudoplatystoma}

En este capítulo aplicaremos los resultados obtenidos en capítulos anteriores sobre la conformación de los patrones en un mecanismo RD para aplicarlos a un sistema concreto, a saber, los patrones que aparecen en la piel de los peces surubíes. Para ello utilizaremos la reacción BVAM. No obstante a que nuestra investigación no es exhaustiva, creemos que ella nos brinda herramientas valiosas para buscar los mecanismos que modelen dichas estructuras.

\subsection{Antecedentes}

\subsubsection{Morfología de los surubíes}

Pseudoplatystoma es un grupo de bagres neotropicales de la familia Pimelodidae que viven en diversos hábitats de Sudamérica tales como los grandes ríos, lagos y bosques inundados del neotrópico. Su pigmentación es distintiva y consiste de bandas verticales oscuras y pálidas, bandas reticuladas y manchas circulares oscuras, según la especie. También se distinguen por la cabeza algo comprimida de donde proviene su nombre ${ }^{1}$. Los individuos de este género son generalmente reconocidos con los nombres vernáculos de: "Bagre Rayado", "Pintadillo", "Surubí" y "Caparari". Se alimentan de otros peces como también miembros de su propia clase y crustáceos, además de algunas frutas y semillas. Su distribución geográfica incluye grandes cuencas del

\footnotetext{
${ }^{1}$ La etimología provienen del griego: pseudo, por falso; platis, plana, stoma, boca.
} 

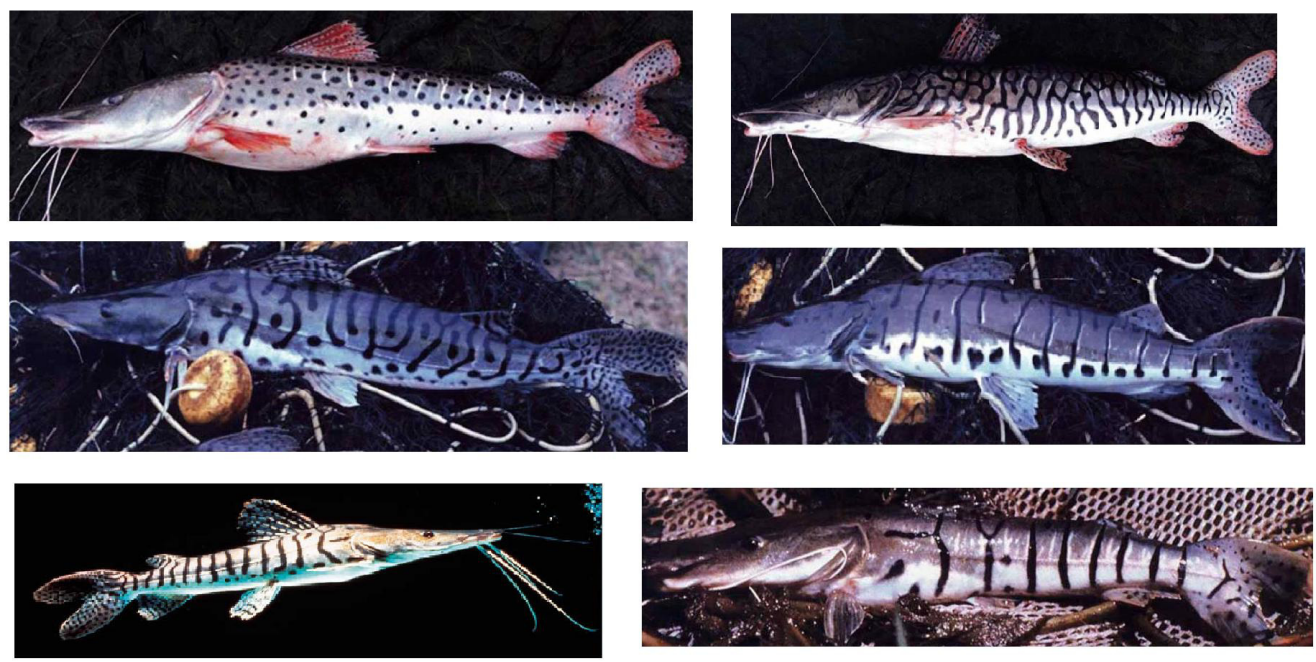

Figura 6.1: Especies de surubíes. Izquierda: Especies Pseudoplayistoma Bleeker. De arriba a abajo P. coruscans, P. fasciatum y P.tigrinum . Derecha: $P$. reticulatum, $P$. orinocoense y $P$. magdaleniatum. Tomado de [45].

neotrópico, el Amazonas, el Orinoco, el Paraná (incluyendo el río Uruguay), ríos de la región de las Guayanas, San Francisco y Magdalena [44].

La sistemática ${ }^{2}$ de este pequeño grupo es totalmente desconocida pues sus especies presentan una gran variación geográfica en su morfología y coloración. Las tres especies mayormente conocidas son las llamadas Pseudoplayistoma Bleeker claramente discernibles por su pigmentación: la región lateral de las poblaciones de $P$. fasciatum presenta bandas verticales oscuras; las de $P$. tigrinum presentan bandas reticuladas y las de $P$. coruscans presentan puntos grandes y dispuestos en hileras en la región lateral del cuerpo. Ver Figura 6.1. Además existen otras cinco especies organizadas y descritas recientemente: $P$. punctifer, $P$. reticulatum, $P$. orinocoense, $P$. metaense y $P$. magdaleniatum [45].

En general, la información que existe sobre la formación de los patrones y de su diversidad en estas especies es muy poca. Por ejemplo, se sabe que la pigmentación comienza a notarse a los 9 días de edad. Esta aparece, en primer lugar, en la parte de la cabeza, siendo hasta el décimo día que se definen las características generales de la coloración. Ver Figura 6.2. También se cree

\footnotetext{
${ }^{2}$ En biología, por sistemática se entiende la clasificación de las especies con arreglo a su historia evolutiva (filogenia).
} 


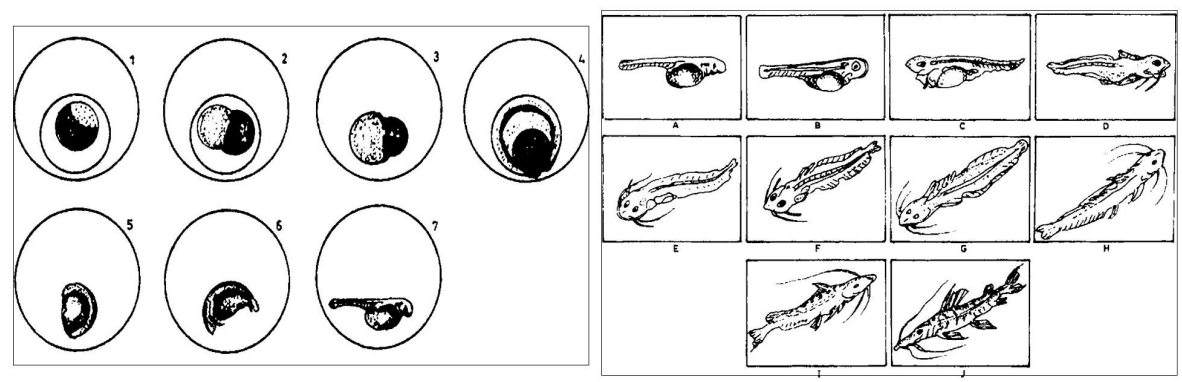

Figura 6.2: Izquierda: Estados de desarroollo del embrión. Desde que el huevo es fecundado hasta la aparición de la larva y la eclosión transcurren alrededor de 14 horas. Derecha: Estadios de desarrollo de las larvas. Es hasta el noveno día que se empieza a notar pigmentación, principalmente en la cabeza.

que el patrón peculiar de coloración inicial de las post-larvas se conserva durante dos meses y medio hasta tomar su coloración definida. Este patrón sirve a los peces para camuflarse como una defensa cuando son arrastradas a las riberas inundadas de los ríos, en donde la vegetación les proporciona sitios ideales para su ocultamiento. En estos lugares, se desarrolla una elevada productividad de zooplancton, quironómidos, de larvas de insectos y de otros organismos que son susceptibles de ser consumidos por las post-larvas y larvas juveniles de Pseudoplatystoma. Asimismo, se ha encontrado que individuos Pseudoplatystoma fasciatum menores de $10 \mathrm{~cm}$, capturados en los ambientes antes descritos, estaban llenos de insectos acuáticos y de larvas de peces. A partir de ello, se puede aseverar que la característica de la aparición del patrón de coloración definitiva marcaría el inicio de los hábitos piscívoros de esta especie [46].

\subsubsection{Modelos de patrones en peces}

Los peces teolostei, así como la mayoría de los vertebrados derivados de una cresta neural, exhiben una gran diversidad de patrones de pigmentación. Estos patrones dependen de células pigmentadas que tienen su origen en la cresta neural $^{3}$. Los patrones de la mayoría de los peces se derivan de tres

\footnotetext{
${ }^{3}$ Una población transitoria de células que se producen durante la neurulación a través del tubo dorsal neural. Las células que integran ésta se dispersan a través del embrión transportando con ello no solamente células de pigmentos, sino otras células que contribuyen al arreglo y formación de otras células y órganos.
} 
tipos de células de pigmentación o cromatóforos: los xantóforos amarillos o naranja; los melanóforos negros; y los iridóforos plateados ${ }^{4}$. La células de pigmento migran hacia toda la piel como una perturbación oscilatoria hasta que se localizan sobre la piel. Sólo cuando la migración ha terminado es que la células adquieren su pigmento característico. De este modo, las regiones de piel son dominadas por un color específico cuando hay acumulación de células de algún tipo específico produciendo así un patrón ${ }^{5}$.

Los mecanismos que establecen el tiempo y la organización de estos patrones aún no se han determinado en forma concluyente. Fueron Kondo y Asai [49] los primeros en sugerir que un mecanismo reacción difusión como el que propuso Turing puede dar cuenta de este proceso. La idea es que un mecanismo RD provee de un pre-patrón químico entre los diversos cromatóforos; este patrón es fijado mediante algún morfogen; finalmente, mediante diferenciamiento celular entre regiones de preponderancia de uno u otro cromatóforo, se da lugar a un patrón en el pez. Los resultados de este y otros trabajos $[50,51,52]$ son imágenes que concuerdan bien con las características de desenvolvimiento, crecimiento y mutación de los patrones en los peces.

Recientemente, Barrio et. al. [53] sugirieron una posible conexión entre un mecanismo RD usando la reacción BVAM y los patrones de los peces surubíes. Nuestro propósito es profundizar en este modelo para encontrar algunos patrones de los peces Pseudoplatystoma. Ver Figura 6.3.

\subsubsection{El modelo BVAM}

Barrio propuso un modelo de reacción difusión para dos componentes químicas $u$ y $v$ suponiendo un punto fijo en la concentración de equilibrio en $\left(u_{0}, v_{0}\right)=(0,0)$ y haciendo un expansión de Taylor de la cinética química alrededor de este punto hasta orden cúbico. En su forma adimensional, este modelo puede escribirse como:

$$
\begin{aligned}
& \frac{\partial u}{\partial t}=D \nabla^{2} u+\eta\left(u+a v-C u v-u v^{2}\right) \\
& \frac{\partial v}{\partial t}=\nabla^{2} v+\eta\left(b v+h u+C u v+u v^{2}\right)
\end{aligned}
$$

\footnotetext{
${ }^{4}$ Nosotros creemos que las franjas y manchas como las del Pseudoplatystoma se deben fundamentalmente a la interacción de los dos últimos tipos de células.

${ }^{5}$ La mayor parte de esta sección procede de los artículos [47, 48]
} 

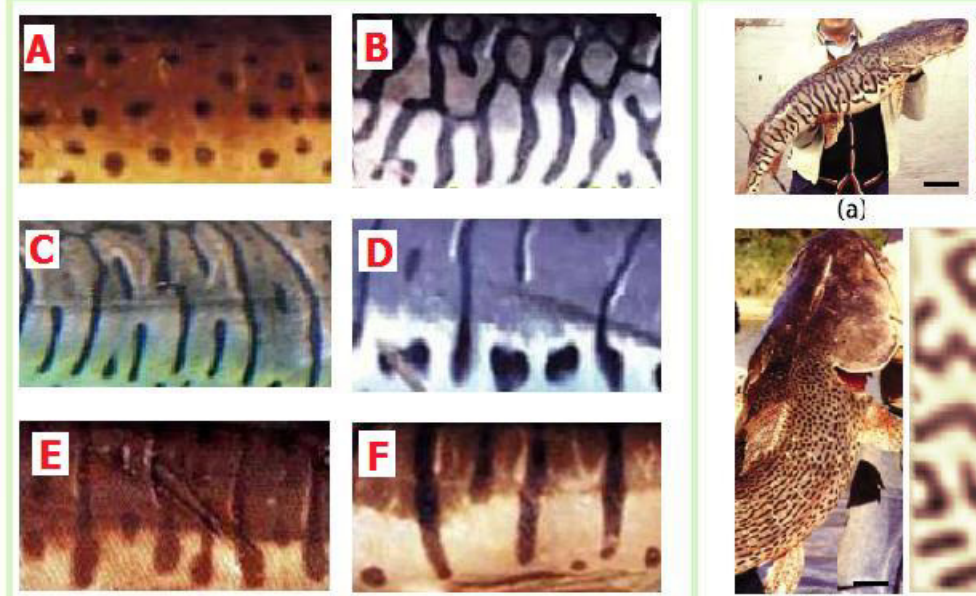

(a)

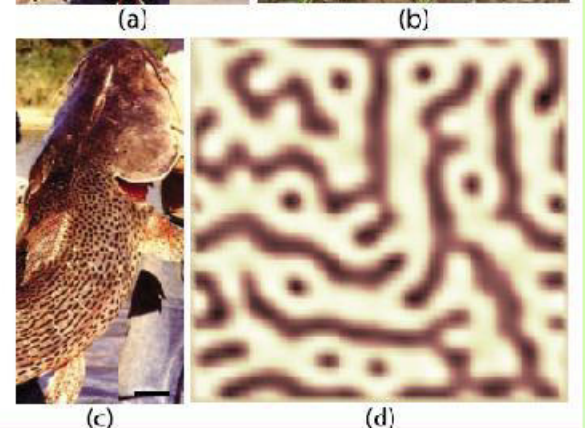

(d)

Figura 6.3: Izquierda: Detalle de los patrones en el flanco de los Pseudoplatystoma. Derecha: Imagen tomada de Barrio [53]. Se sugiere que un mecanismo $\mathrm{RD}$ puede dar lugar a patrones parecidos a los de estos peces.

donde $D=D_{u} / D_{v}$ es el cociente de constantes de difusión, $\eta$ proporciona la escala espacio-temporal, y $\mathrm{C}$ es una medida de la fuerza de la interacción cuadrática. Además del origen, el modelo tiene otros dos puntos fijos ubicados en $\left(u_{0}, v_{0}\right)=v_{ \pm}(-g, 1)$, con:

$$
v_{ \pm}=\frac{-C \pm \sqrt{C^{2}+4 f}}{2}
$$

y los parámetros $g$ y $f$ definidos como $g=(a+b) /(1+h)$ y $f=b / g-h$. Para estos tres puntos fijos, el jacobiano toma la forma general:

$$
\mathbb{J}=\left(\begin{array}{cc}
1-\phi & a-\psi \\
h+\phi & b+\psi
\end{array}\right),
$$

con $\phi=v_{0}\left(c+v_{0}\right)$ у $\psi=C u_{0}+2 u_{0} v_{0}=-g v_{0}\left(C+2 v_{0}\right)$. Si definimos la matriz de difusividades como aquella cuya traza es la difusividad de $u$ y de $v$, es de$\operatorname{cir}, \mathbb{D}=\operatorname{tr}(D, 1)$, entonces el sistema linearizado puede analizarse estudiando los eigenvalores $\lambda_{k}$ de la matriz $\mathbb{J}-k^{2} \mathbb{D}$. Un estudio detallado del modelo ha sido publicado en otro lugar [54] confirmando que la existencia de otros puntos fijos le da al modelo una gran riqueza de comportamientos; además, las simulaciones numéricas confirman gran variedad de patrones estacionarios y 


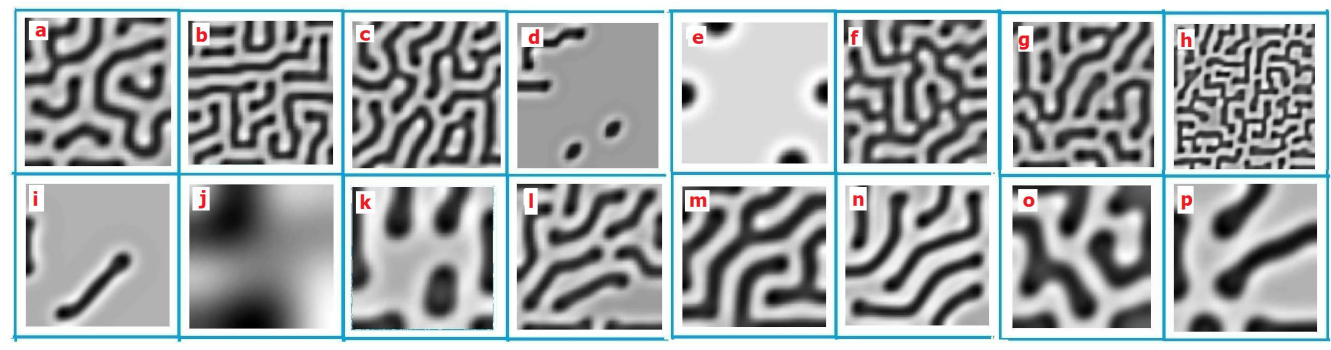

Figura 6.4: Patrones (no necesariamente estacionarios) de la reacción BVAM en un cuadrado de lado 80 después de un tiempo $\mathrm{T}=750$. Las condiciones iniciales son aleatorias en todo el dominio y las condiciones de frontera son de flujo nulo. Se utilizaron los valores de $f=0.65, g=0.165$ y $h=-2.5$. Los valores de los parámetros $(D, C, \eta)$ están dados de izquierda a derecha y de arriba a abajo por: a $(0.344$; $0.72 ; 0.225), \mathrm{b}(0.172 ; 2.0 ; 0.1), \mathrm{c}(0.172 ; 0.8 ; 0.15), \mathrm{d}(0.344 ; 1.5 ; 0.3), \mathrm{e}(0.516 ; 0.72$; $0.15), \mathrm{f}(0.172 ; 0.00 ; 0.15), \mathrm{g}(0.172 ; 0.72 ; 0.15), \mathrm{h}(0.172 ; 3.5 ; 0.15), \mathrm{i}(0.344 ; 1.55$; $0.15), \mathrm{j}(0.344 ; 1.7 ; 0.15), \mathrm{k}(0.344 ; 0.72 ; 0.075), \mathrm{l}(0.344 ; 0.0 ; 0.225), \mathrm{m}(0.344 ; 0.0$; $0.15), \mathrm{n}(0.344 ; 1.4 ; 0.15), \mathrm{o}(0.516 ; 0.0 ; 0.15), \mathrm{p}(0.516 ; 0.36 ; 0.15)$. Las simulaciones se hicieron mediante Elemento finito con una malla de 3200 triángulos y un paso de tiempo $\Delta t=5 \times 10^{-2}$.

transitorios en la reacción BVAM. De particular interés para nosotros son las franjas y puntos desordenados, pues son este tipo de formas y combinaciones de ellas las que buscamos para los patrones de los Pseudoplatystoma.

\subsection{Simulaciones numéricas}

A diferencia de los patrones convencionales obtenidos de la reacción BVAM (ver Figura 6.4), los patrones en los peces Pseudoplatystoma presentan: 1) distinto grosor entre las franjas negras y plateadas (salvo en el P. coruscans); 2) una orientación preferencial de las franjas con la dirección vertical, y 3) una variación de los patrones en la dirección vertical, por ejemplo, las motas que se presentan sólo en la parte de abajo del $P$. oricoense o el reticulado en la parte superior del $P$. reticulatum.

Esto quiere decir que, a pesar de que el modelo BVAM convencional reproduce el ordenamiento irregular de franjas y puntos que presentan estos peces, aún no explica la dependencia espacial de las formas y su orientación. Con el modelo convencional nos referimos al uso de 1) fronteras fijas, 2) do- 
minio cerrado, 3) coeficientes constantes y 4) condiciones iniciales aleatorias en todo el dominio alrededor del punto de equilibrio. En este trabajo exploraremos como es que la variación en uno o varios de estos factores ${ }^{6}$ contribuye a la disposición especial de los patrones en cada especie de Pseudoplatystoma.

\subsubsection{Condiciones iniciales y de frontera}

A pesar de la mucha atención que han tenido los sistemas reacción difusión en los últimos años, es de notarse que el principal estudio se ha enfocado en el sistema de ecuaciones y en los parámetros que son capaces de producir patrones espaciales estacionarios. Sin embargo, poco se ha estudiado sobre la influencia de las condiciones iniciales y de frontera, los cuales completan el problema y determinan las posibles soluciones.

Como hemos visto en el capítulo anterior, las condiciones iniciales utilizadas con más frecuencia en este tipo de problemas consisten en fijar las concentraciones de ambos reactantes en sus puntos de equilibrio y agregar una contribución aleatoria, de tal manera que el valor en cada nodo no esté correlacionado con los valores en los nodos adyacentes (Ver por ejemplo $[55,39,41])$. En este caso, la orientación preferencial de las formas en los patrones puede producirse mediante dos formas: 1) utilizando fronteras tipo Neumann que alinean las franjas o las celdas paralelas o perpendiculares a las fronteras, o 2) agregando alguna dirección preferencial en las condiciones iniciales $[34,38,39]$. Nuestro interés en esta sección del trabajo es mostrar dos cosas: 1) qué estas dos condiciones podrían producir la orientación vertical que buscamos para las franjas de los peces Pseudoplatystoma, y quizá más importante 2) que variaciones en las condiciones iniciales pueden producir patrones muy distintos (a pesar de usar el mismo conjunto de parámetros), no sólo en cuanto a la orientación, sino a la disposición de las formas que constituyen el patrón.

La influencia de las condiciones iniciales en el modelo, se muestra en la Figura 6.5, mediante la gráfica de algunos resultados. En dicha figura se presenta la evolución temporal del patrón presentando en la columna de la izquierda la condición inicial, en las columnas intermedias algunos estados transitorios, y en la columna de la derecha el patrón estacionario. Estos resultados se obtuvieron manteniendo fijos: el dominio, las condiciones de

\footnotetext{
${ }^{6}$ Explotaremos solamente los últimos tres, pues el el crecimiento del dominio requiere de mayor cuidado, además de que ya ha sido estudiado previamente por $[50,52,58]$.
} 
frontera (Neumann, flujo nulo) y el conjunto de parámetros. Es decir, solo se modifica la condición inicial en cada caso. Ver Figura 6.5 para detalles.

- Primero comparamos las filas 6.5.A y 6.5.B donde hemos utilizado condiciones aleatorias convencionales, con la única diferencia de que la intensidad del ruido es distinta, pues mientras el 6.5.A los valores de $u$ y $v$ están entre \pm 0.001 , en 6.5 .B están entre \pm 0.1 . Los resultados muestran que las líneas que conforman el patrón son (en promedio) más largas en el primer caso que en el segundo, donde incluso aparecen manchas más parecidas a puntos que a franjas propiamente.

- Ahora comparemos las filas 6.5.C y 6.5.D. En ambos casos las concentraciones de ambos reactantes son cero excepto en una pequeña franja en el lado izquierdo del cuadrado. En el primer caso, la franja está dada por $u=-0.1$ y $v$ aleatorio, mientras que en el segundo caso ambos son aleatorios ${ }^{7}$. En ambos casos puede observarse que la perturbación avanza como una onda hasta alcanzar el otro lado del dominio. Sin embargo, el frente de onda de 6.5.C avanza como una franja vertical, que se duplica y rebota en la frontera vertical derecha para formar una figura del tipo laberíntico. En el segundo caso 6.5.D la perturbación avanza desordenadamente, incluso con alguna preferencia por la dirección horizontal, dando al final un patrón más bien parecido a 6.5.B.

- Comparemos las figuras 6.5.E y 6.5.F. En este caso la concentración de ambos reactantes es cero en casi todo el dominio, salvo en una franja en la parte superior del dominio. En el primer caso, esta franja contiene cuatro oscilaciones cosenoidales de $u$ mientras que el otro tiene sólo tres. Estas oscilaciones son de amplitud \pm 0.05 . La variable $v$ es aleatoria en esta franja y cero fuera de ella. En el caso de 6.5.E las franjas avanzan hasta convertirse en manchas con más o menos la misma disposición de columnas, mientras que en el caso 6.5.F las franjas avanzan hasta dispersarse y formar un patrón más parecido a 6.5.C.

- Finalmente presentamos un patrón donde las franjas se encuentran completamente ordenadas, como se muestra en la Figura 6.5.G. Las condiciones iniciales son oscilaciones verticales con exactamente cuatro mínimos, más ruido aleatorio para ambas variables. Las oscilaciones son

\footnotetext{
${ }^{7}$ En todas la simulaciones siguientes, la intensidad de la aleatoriedad se ajustará a \pm 0.05 , a menos que se indique lo contrario.
} 


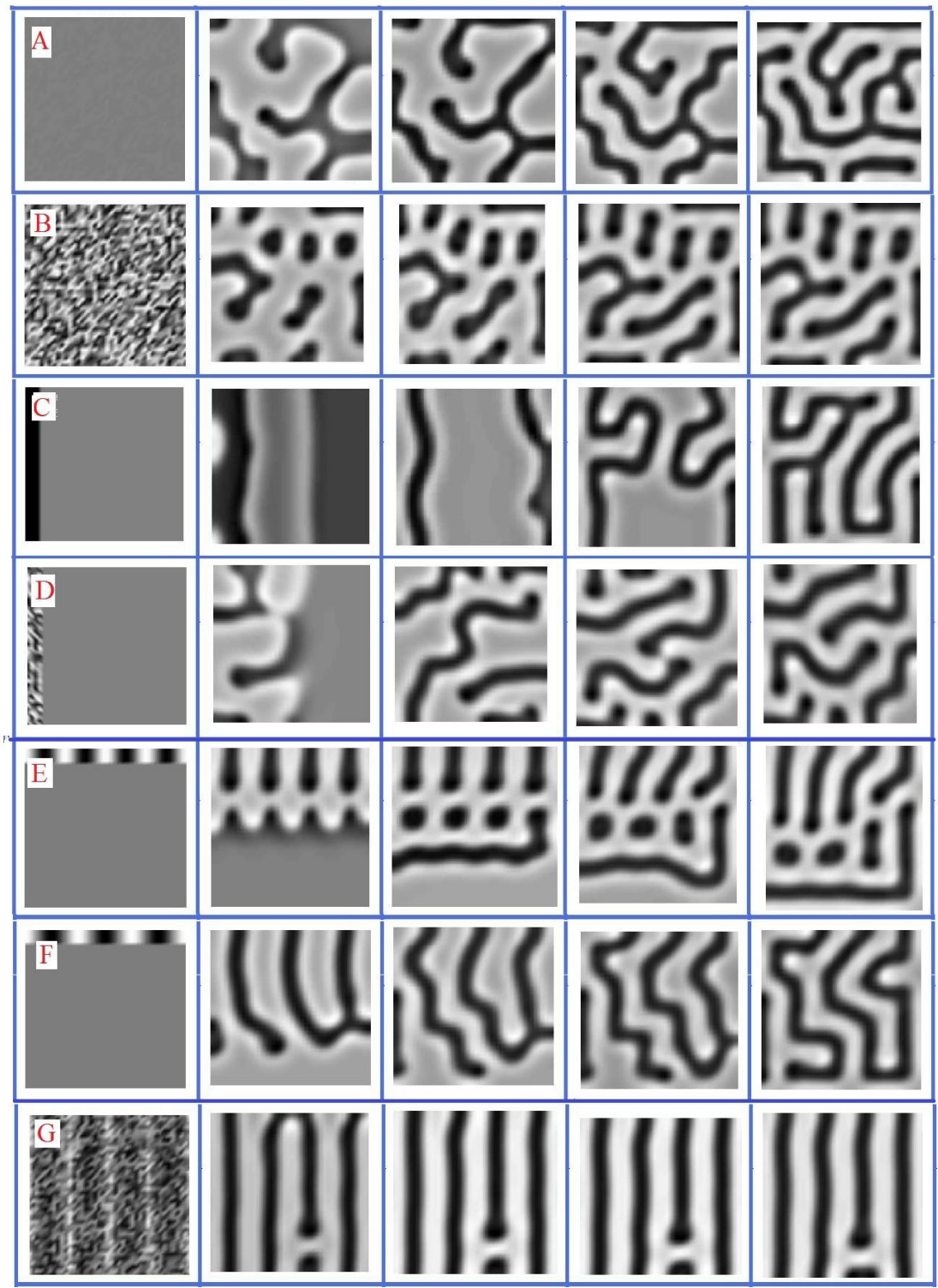

Figura 6.5: Diferencia de patrones variando solamente las condiciones iniciales. En la columna de la izquierda aparece la condición inicial, en las columnas intermedias algunos estados transitorios, y en la derecha el patrón estacionario obtenido después de un tiempo: A)875, B)875 C)5000, D)875, E)5000, F)4000, G)500. El dominio es igualmente un cuadrado de lado 80 y los parámetros de la reacción se fijaron en $f=0.65, g=0.165, h=-2.5, D=$ $0.344, C=0.72$ y $\eta=0.15$ para todos los casos. Las simulaciones numéricas se realizaron usando el método de elemento finito con los mismos parámetros de discretización que la figura anterior. 
cosenos verticales de amplitud 0.05 en todo el dominio. La orientación vertical casi perfecta se desvanece cuando uno utiliza algún un número de oscilaciones en las condiciones iniciales distinto de cuatro. En este caso, las franjas se desvanecen formando patrones más bien parecidos a 6.5.D sin orientación preferencial alguna.

De nuestro análisis podemos deducir que

- las fronteras Neumann tienden a alinear las formas con los bordes,

- las formas del patrón pueden viajar como una perturbación y conservarse o desvanecerse dependiendo de la condición inicial, y que

- una de las formas de conseguir la orientación puramente vertical puede ser a través de condiciones iniciales que de alguna manera anticipe el patrón.

El problema de esta última aseveración es que para explicar el pre-patrón químico vertical tendríamos que apelar a un patrón previo en las condiciones iniciales, el cual se tendría que justificar a su vez ya sea por otro pre-patrón o por algún otro mecanismo ajeno al modelo.

Otro aspecto a considerar es el tipo de condiciones de frontera a utilizar. Convencionalmente se han utilizado condiciones periódicas y de flujo nulo para demostrar la existencia de patrones. En nuestro caso, atendiendo a la forma de pez pseudoplatystoma, consideramos que también podría ser relevante un dominio con flujo nulo en las fronteras horizontales (es decir, que no hay flujo hacia arriba o a hacia abajo) y condiciones periódicas en los bordes verticales atendiendo a que el patrón se repite espacialmente a lo largo del pez desde el final de la cabeza (opérculos) hasta la raíz de la cola (aleta caudal). No obstante, estas condiciones no deben verse como restrictivas puesto que el uso de los distintos tipos de condiciones de frontera que implementamos no altera significativamente el patrón [39, 38]. Ver Figura 6.6.

\subsubsection{Coeficientes dependientes del espacio}

En esta sección del trabajo nos concentraremos básicamente en la obtención de las distintas formas (puntos, líneas, manchas) como resultado de un solo proceso. En otras palabras, exploraremos cómo una variación espacial en los parámetros de la reacción química puede dar lugar a que aparezcan 


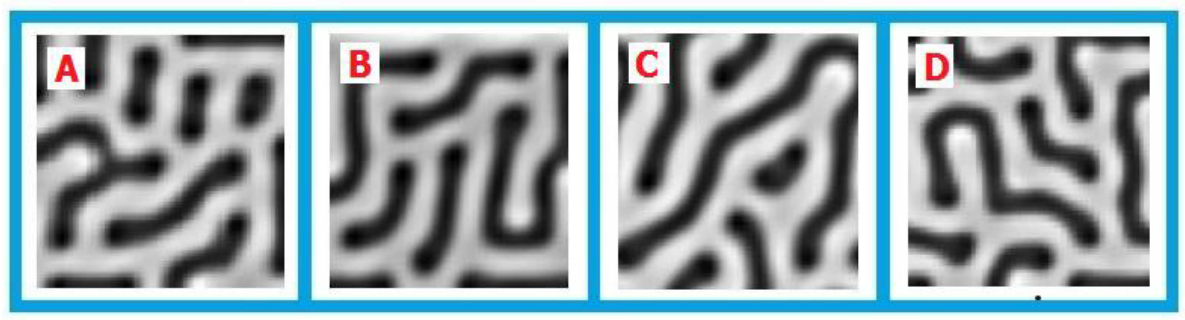

Figura 6.6: Patrones variando únicamente las condiciones de frontera: A) Neumann, flujo nulo, B) Neumann, con una fuente de intensidad 0.05 en la frontera superior, C) periódicas y D) Izquierda y derecha son periódicas, y flujo nulo arriba y abajo. Los patrones son tomados después de un tiempo $\mathrm{T}=350$. Los patrones fueron obtenidos con el método SBDF en un cuadrado de lado 80 , un paso de tiempo $\Delta t=0.05$ y una malla de $56 \times 56$ nodos.

grecas, franjas, y puntos en la misma imagen. Para ello, atenderemos a variar los coeficientes $\eta$ y $C$ de la reacción BVAM. Como Barrio observó, todas estas formas pueden obtenerse variando únicamente el coeficiente cuadrático $C$ (Ver Figura 6.7). Además, es bien sabido que en la forma adimensional de una reacción química, el valor $\eta$ es un factor de escala espacio-tiempo proporcional a $L^{2} / t$, por lo que si se fija el tamaño de dominio, un aumento en $\eta$ representa una disminución del tamaño en la forma del patrón y una mayor velocidad de reacción ${ }^{8}[32]$.

Los resultados de nuestros experimentos al hacer variaciones en estos coeficientes, así como los detalles de las simulaciones numéricas se encuentran en la Figura 6.8, que procedemos a analizar:

- En la primera columna de la Figura 6.8 exploramos el efecto del parámetro $\eta$ en un patrón de manchas (a), de franjas (b) y de puntos (c) correspondientes a las Figuras 6.7A, B y D respectivamente donde $\eta=0.35$ era fijo. La diferencia es que ahora $\eta$ varía, aumentando su valor linealmente desde 0.15 en la parte de abajo, hasta 0.40 en la parte superior ${ }^{9}$. Se observa que un aumento en $\eta$ provoca disminución en el tamaño en

\footnotetext{
${ }^{8}$ También se ha probado que una difusión anisotrópica puede generar orientaciones específicas de los patrones [56, 57]. Estos modelos suponen que cada una de las sustancias tienen una dirección preferencial para difundirse, lo cual da una orientación específica al patrón. Nosotros no consideraremos esta variación al modelo.

${ }^{9}$ Formalmente: $\eta(x, y)=0.15+(0.40-0.15) y / L$.
} 


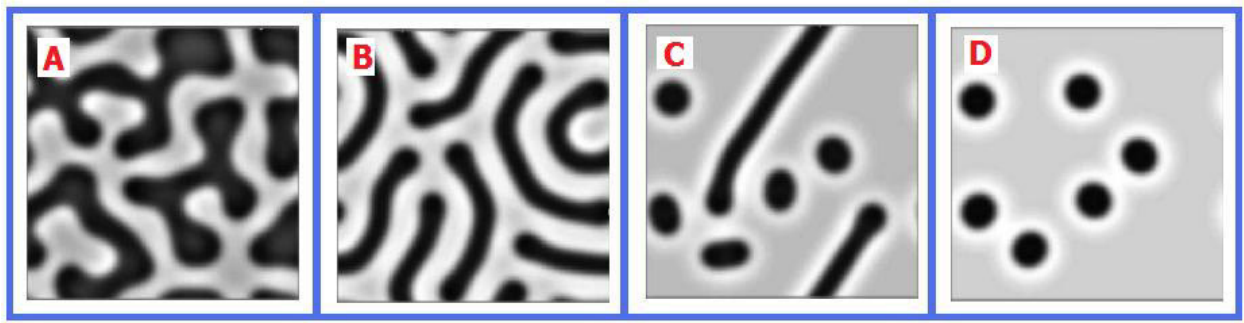

Figura 6.7: Tomada de Barrio [53]. Un cambio en las formas del patrón puede darse mediante el cambio únicamente en el parámetro $C$ : A)0.0, B)0.6, C) 0.71, D)0.72. Los demás parámetros son iguales a los de la figura anterior.

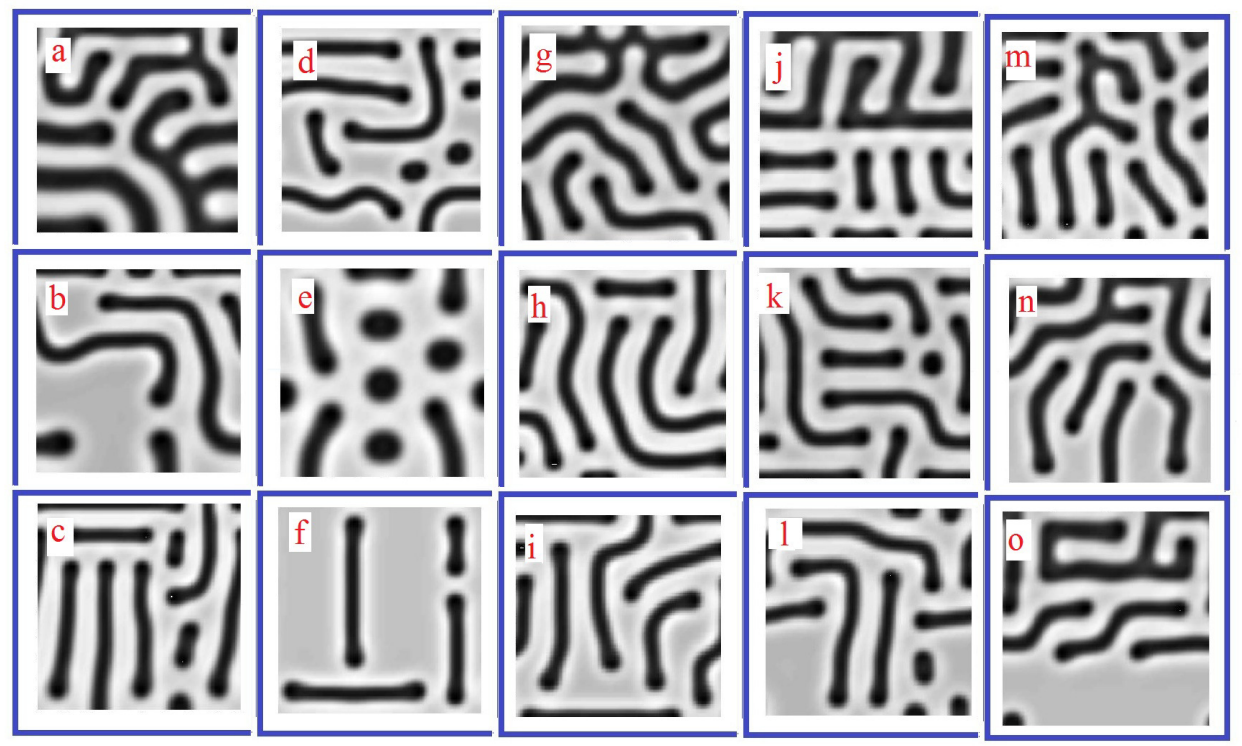

Figura 6.8: Patrones con dependencia espacial de $\eta$ y $C$. Los otros parámetros se fijan en los valores antes dados. Las simulaciones se hicieron usando el método 2-SBDF con condiciones de flujo nulo en las fronteras horizontales y periódicas en las verticales, para un cuadrado de longitud $\mathrm{L}=80$. En todos los casos se usó una malla de 4096 nodos y un paso de tiempo $\Delta t=5 \times 10^{-2}$. 
franjas y manchas sin una modificación en la forma. Sin embargo, en el caso de los puntos, el gradiente de $\eta$ provoca que se conviertan en franjas.

- En la segunda columna, las Figuras 6.8 exploran las oscilaciones cosenoidales del parámetro $C$ en la dirección horizontal. Las oscilaciones de $C$ ocurren entre los valores 0.71 y 0.80 que corresponden a los valores donde había franjas y puntos en la Figura 6.7. La única diferencia es el número de oscilaciones verticales de $C$, siendo éstas 2,3 y 4 respectivamente $^{10}$. Se observa que la orientación vertical se conserva solamente para el caso 6.8.e, mientras que en los otros dos puntos y franjas se entreveran desordenadamente, como en 6.8.d, o aparecen franjas horizontales, como en 6.8.f.

- En la tercera columna de 6.8 exploramos el efecto de aumentar $C$ de manera lineal entre los tres distintos intervalos que hay entre las distintos valores de $C$ que determinan los cuatro diversos comportamientos de la Figura 6.7. Así, 6.8.g daría en principio el paso de manchas a líneas , 6.8.h de líneas a puntos, y 6.8.i constituiría una mezcla líneas y puntos $^{11}$. Sin embargo, al igual que en 6.8.b, el gradiente de $C$ ocasiona que se destruyan las formas que incluyen puntos convirtiéndolos en líneas.

- La cuarta columna es igual que la anterior, salvo que el tránsito no es continuo, sino discontinuo, pues en la mitad superior e inferior se usan dos valores de $C$ dictados por los mismos intervalos que antes ${ }^{12}$. En estos puede verse el traslape de formas y la distorsión de los puntos excepto en el caso 6.8.j.

\footnotetext{
${ }^{10}$ Es decir: $C(x, y)=0.71+(0.80-0.71) / 2(1+\cos (2 \pi m x / L))$ siendo $m$ el número de oscilaciones verticales.

${ }^{11}$ Formalmente: Los cuatro valores de $C$ donde hay un cambio de comportamiento son $C_{1}=0.0, C_{2}=0.60, C_{3}=0.71, C_{4}=0.72$. En cada figura, $C$ aumenta de arriba hacia abajo según $C(x, y)=C_{m+1}+\left(C_{m}-C_{m+1}\right) y / L$, donde $m=1,2,3$.

${ }^{12}$ Es decir: Usando los mismos valores $C_{m}$ de la nota anterior, cada figura está dada por:
}

$$
C(x, y)= \begin{cases}C_{m} & \text { si } y \geq L / 2, \\ C_{m+1} & \text { si } y<L / 2 .\end{cases}
$$


- Finalmente, en la última columna de la Figura 6.8 presentamos combinaciones de los anteriores. El patrón 6.8.m constituye el cambio lineal de $C$ en el intervalo 0 a $0.72^{13} ; 6.8$.n es una mezcla de (g) y (e), es decir, de oscilaciones en la dirección horizontal y aumento gradual en la dirección vertical ${ }^{14}$; y finalmente 6.8.o constituye el mismo cambio que 6.8. $\mathrm{m}$ pero de manera discontinua en tres escalones dados por $0,0.60 \mathrm{y}$ $0.72^{15}$.

De esta sección uno puede notar que la aparición de franjas junto con puntos en el patrón es muy difícil. La razón es que los últimos tienden a estirarse en presencia de un gradiente. Por ello, en la siguiente sección consideramos otra posibilidad para su formación.

\subsubsection{Sistema reacción-convección}

Matemáticamente hablando, la advección representa la variación de un campo escalar por el efecto de un campo vectorial. En términos físicos, la advección es un fenómeno de transporte de una sustancia debida al movimiento de cuerpo del fluido (bulk motion), por ejemplo, cuando el polvo y el limo son arrastrados por la corriente de un río. Técnicamente, la convección es la suma de los efectos de la difusión y la advección cuando ambos fenómenos son comparables. De este modo, la transferencia convectiva de una sustancia se da a través de la difusión (el movimiento aleatorio Browniano de las partículas en el fluido), y por advección (en el cual la materia es transportada por el movimiento a mayor escala de fluido)[59].

En esta sección exploraremos si la verticalidad de las franjas y el ordenamiento de las distintas formas dentro de un patrón pueden obtenerse por medio de un movimiento advectivo de ambos químicos en el sistema RD original. Por ello, si agregamos el término advectivo a la ecuación (2.14) original, el sistema RC (reacción-convección) puede escribirse según [42] como:

\footnotetext{
${ }^{13}$ Es decir: $C(x, y)=0.72+(0.0-0.72) y / L$.

${ }^{14}$ Formalmente: $C(x, y)=(0.60-0.0) y / L+(0.72-0.60) y /(2 L)(1+\cos (6 \pi x / L))$.

${ }^{15}$ Es decir:
}

$$
C(x, y)= \begin{cases}0.0 & \text { si } y \geq 2 L / 3 \\ 0.60 & \text { si } 2 L / 3>y>L / 3 \\ 0.72 & \text { si } y \leq L / 3\end{cases}
$$




$$
\frac{\partial \mathbf{w}}{\partial t}=\mathbf{R}(\mathbf{w})+\mathbb{D} \nabla^{2} \mathbf{w}+\nabla \mathbf{w} \cdot \mathbf{z} .
$$

En el Apéndice B esbozamos la resolución numérica de esta ecuación para el caso particular que utilizaremos, a saber, un movimiento de cuerpo constante en la dirección vertical: $\mathbf{z}=-z \hat{e}_{y}$. En la Figura 6.9 exploramos el efecto de la advección en los patrones originales de la Figura 6.7. Como puede observarse, la advección introduce no solamente la orientación vertical deseada, sino además presenta puntos y franjas en el mismo patrón lo cual no ocurría en nuestro estudio de los coeficientes dependientes del tiempo.

\subsection{Discusión}

Como adelantamos al principio del capítulo, nuestro estudio no es aún concluyente respecto a cómo los factores estudiados junto con la reacción BVAM se coordinan para modelar los patrones que emergen en los peces Pseudoplatystoma ${ }^{16}$. No obstante, creemos que nuestro estudio permite adelantar algunas hipótesis que esperamos corroborar posteriormente:

- En el caso del P. coruscans (Figura 6.10.A), el patrón presenta esencialmente manchas cuasi-circulares que presentan cierto ordenamiento horizontal. No obstante, también pueden observarse algunas manchas alargadas en la parte superior. Esto sugiere que estos patrones pueden modelarse como en la Figura 6.7 con algún tipo de dependencia horizontal en los coeficientes que diera no solamente la regularidad en esta dirección, sino que también distorsionara levemente los puntos, tal y como hemos observado en la sección anterior. Si bien nuestro estudio del sistema RC nos indica que la advección alarga algunos puntos, tampoco podemos descartar que una advección más lenta junto con condiciones iniciales como a las de la Figura 6.5 puedan producir este patrón.

\footnotetext{
${ }^{16}$ La limitación es de carácter temporal. Cada simulación requiere de por lo menos un día de ejecución computacional. Además de que los patrones que buscamos son muy complejos, por lo que su modelación requiere de la combinación de los varios factores que hemos estudiado, combinaciones éstas que seguiremos intentando aunque ya no entren dentro del cuerpo de este trabajo.
} 


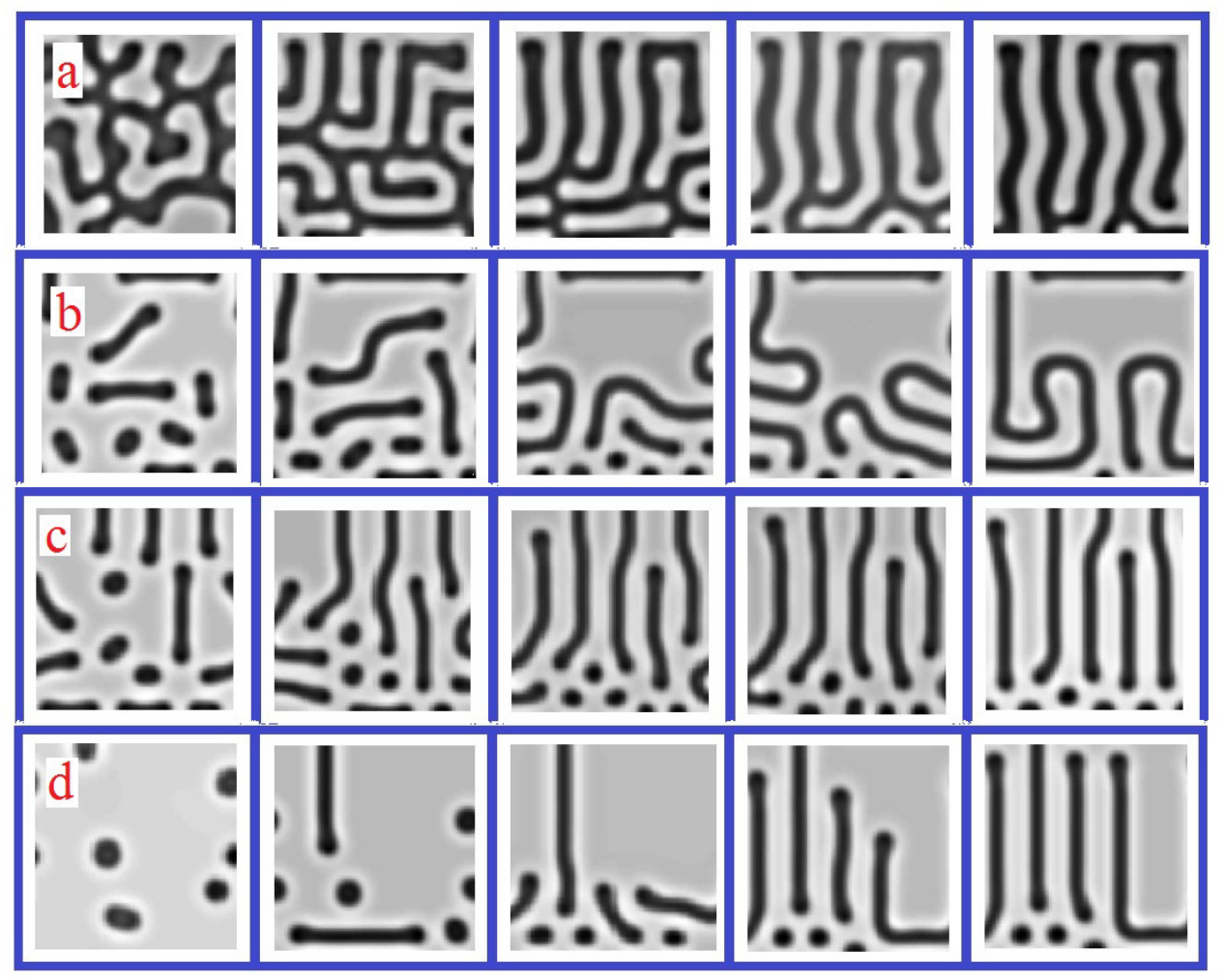

Figura 6.9: Los mismos patrones que en Figura 6.7 pero con el efecto de la advección en la dirección vertical: $\mathbf{z}=-0.02 \hat{e}_{y}$. La primera columna muestra condiciones análogas a los sistemas sin advección, pero conforme avanza el tiempo (hacia la derecha en cada fila), los patrones obtienen la orientación vertical. La columna más hacia la derecha muestra el patrón estacionario. Las simulaciones fueron generadas por una variación del método 2-SBDF esbozado en el Apéndice B con los mismos parámetros de discretización que la figura anterior. 
- En el caso del P. reticulatum (Figura 6.10.B), el patrón consiste de un patrón reticulado en la parte superior para luego tornarse en líneas verticales. Es posible que una dependencia espacial del parámetro $C$ parecido a la Figura 6.8.n, o alternativamente una dependencia como en 6.8.g junto con el efecto de la advección (Figura 6.9.a) pueden dar lugar a este patrón.

- En el caso del P. tigrinum (Figura 6.10.C), el patrón consiste de líneas casi verticales entrelazadas y con puntos que llenan los diversos huecos. Se nos ocurren las posibilidades de una dependencia espacial como en Figura 6.8.f y/o el hecho de que la configuración del patrón biológico (visible) no sea necesariamente el patrón químico estacionario ${ }^{17}$. En este caso, patrones transitorios como en la Figura 6.9.b o (c), junto con una advección menos marcada pueden dar lugar al patrón de esta especie.

- En los casos del P. oriconoense y P. magdalenatium (Figuras 6.10.D y (F) respectivamente), el patrón consiste de líneas verticales bien separadas con puntos en la parte inferior, siendo el tamaño de los puntos más grandes en la primera especie. Una posibilidad para este tipo de patrón sería una modificación en las condiciones iniciales como en la Figura 6.5.E; o quizá, otra posibilidad más plausible es que los patrones sean formados por efectos advectivos como en las Figuras 6.9.c o (d).

- En el caso del P. fasciatum (Figura 6.10.E) encontramos patrones de líneas verticales con puntos en los espacios. Estos patrones los podemos identificar con el patrón de coeficientes espacio-dependientes del tipo de la Figura 6.8.e combinado con (c); la primera dependencia establecería

\footnotetext{
${ }^{17}$ Creemos importante remarcar el hecho de que las hipótesis que hemos hecho respecto a la formación de patrones en los animales no descartan esta posibilidad. El diferenciamiento celular (que produce células de un color o de otro) se activaría en algún momento de la reacción química (no necesariamente hasta que la reacción alcance el equilibrio). El desarrollo de los patrones biológicos como función del tiempo podría independizarse del desarrollo de la reacción química (el patrón químico) suponiendo que los morfógenos sean distintos de los cromatóforos, en este caso, es decir, que los químicos que activan el diferenciamiento celular sean distintos de las sustancias químicas que producen el patrón. En este caso, un mecanismo RD como el de Turing establecería solamente un patrón inicial y otro tipo de proceso biológico determinaría la evolución del patrón visible en el animal. Esto, por supuesto, también es una hipótesis.
} 
el ordenamiento de líneas y puntos, mientras que la segunda produciría el cambio de tamaño del grosor de las franjas.

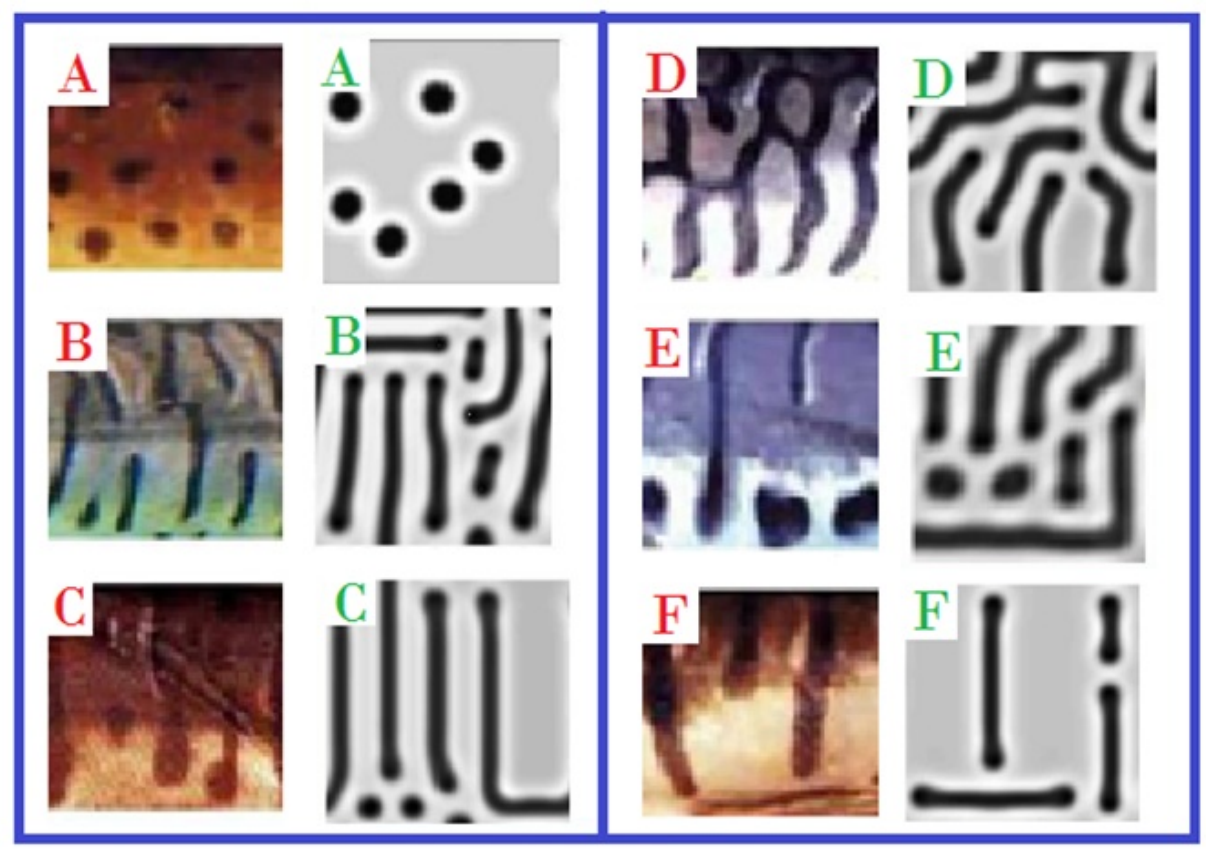

Figura 6.10: Comparación entre los patrones encontrado en los flancos de los diversos surubíes (en rojo) y los encontrados en simulaciones numéricas (en verde). Ver texto para detalles.

Si bien nuestro estudio no es concluyente respecto a estas ideas, permite suponer que la formación de patrones en la naturaleza, aun suponiendo válido el modelo de Turing, es mucho más compleja que lo se supone usualmente en estos modelos. Esperamos que nuestro análisis sirva como motivación para iniciar un estudio más profundo que permita fijar nuevas bases para entender que la irregularidad de los patrones en los animales, la diversidad biológica entre organismos de la misma especie, la evolución del patrón en un mismo individuo, etc., dependen no sólo de un mecanismo reacción difusión, sino que también son cruciales el cómo y cuándo se activa el diferenciamiento celular, cómo depende del dominio en cuestión y cómo podría relacionarse con otros mecanismos como fuentes, advección o gradientes. 


\section{Conclusiones y Perspectivas}

Entre las principales conclusiones de este trabajo se encuentran:

- Un patrón de Turing es una forma que aparece como el resultado de una reacción oscilatoria entre dos o más especies químicas y la difusión molecular diferenciada. Aunque ambos mecanismos por separado sean homogeneizadores, bajo ciertas combinaciones de parámetros un mecanismo RD da lugar a patrones especiales estacionarios.

- Un patrón químico de este tipo puede servir para explicar los patrones que aparecen en la piel de los animales. Según la hipótesis de Turing, el patrón biológico puede aparecer a consecuencia de un pre-patrón químico. Si las células tienen agentes capaces de registrar la concentración exterior donde se ha formado un patrón químico, entonces pueden diferenciarse de acuerdo a éste, dando como resultado células de un color y de otro, formando el patrón biológico.

- Es posible establecer una definición puramente matemática de los patrones de Turing como un mecanismo RD en el cual: 1) El estado de equilibrio se determina unicamente de la cinética química y es estable en ausencia de difusión, y 2) la inclusión de la difusión introduce modos inestables en la solución que son los que ocasionan formas espaciales que no se desvanecen en el tiempo.

- Dada la complejidad de las ecuaciones RD, es necesario resolverlas numéricamente. Las estrategias más comunes para resolver este tipo de ecuaciones son los métodos de diferencias finitas y el de elemento finito. Nuestro trabajo confirma que los métodos explícitos, semi-implícitos 
de primer y segundo orden, el esquema de direcciones alternantes y el método de elemento finito son eficaces para la solución 1D y 2D de este tipo de ecuaciones.

- Cada esquema o método tienen sus ventajas y desventajas en cuanto a la facilidad de programación, tiempo de ejecución y precisión. Sin embargo, bajo ciertas condiciones sobre el mallado y el paso de tiempo, todos los métodos que utilizamos ofrecen los mismos patrones. Si bien este trabajo no esta enfocado en la eficiencia y desempeño de los métodos, es bien sabido que la elección de uno u otro depende del tipo de ventaja que uno desee explotar.

- El modelo BVAM presenta una gran diversidad de comportamientos entre los que se incluye la existencia de patrones estacionarios irregulares fuera de la región de Turing. En particular la existencia de líneas y puntos desordenados en este modelo pueden utilizarse para modelar los patrones de los peces Pseudoplatystoma.

En cuanto al trabajo futuro, nuestra investigación tiene las siguientes perspectivas:

- Es necesario hacer más simulaciones numéricas para poder encontrar con todo detalle los patrones que se encuentra en la piel de los peces Pseudoplatystoma. Aunque nuestro trabajo sienta los principales mecanismos que podrían intervenir en el estudio de estos patrones, un estudio exhaustivo de este tema requerirá explorar más combinaciones de parámetros, dependencias espaciales en otros coeficientes como el de difusión, el efecto del cambio de tamaño de los peces y estudiar con más detalle el papel de la advección, de fuentes o de otros mecanismos.

- Las ecuaciones del tipo reacción difusión no están limitadas al campo de los patrones biológicos, sino que han sido encontradas en muchos sistemas, dos de los cuales me parecen de particular interés. El primero tiene que ver con la propagación de calcio en distintos tipos de células, donde ha sido comprobado que el $\mathrm{Ca}^{2+}$ intercelular reacciona químicamente con el calcio almacenado en diversos organelos de la célula al mismo tiempo que se difunde. Esto produce ondas de $\mathrm{Ca}^{2+}$ que se propagan en el interior de la célula activando otras funciones celulares importantes como la secreción y la comunicación intercelular [60]. Creemos que se puede relacionar la dependencia espacial y temporal de la 
secreción con la activación de ondas de calcio por un mecanismo RD, por lo que pretendemos realizar el estudio numérico correspondiente para comprobar esta hipótesis.

- El segundo sistema reacción difusión que nos interesa está relacionado con los cristales líquidos (LQ). En estos sistemas se ha predicho recientemente la existencia de patrones y ondas viajeras entre la densidad del sistema y el parámetro de orden (un número que mide qué tan ordenados están los cilindros que conforman un sistema LQ) [61]. Aunque las ecuaciones que describen este sistema no son exactamente del tipo RD, existen muchas analogías con los sistemas vistos en esta tesis, por lo que creemos poder determinar las condiciones en las que estos patrones aparecen y, de esta forma, reproducir efectos físicos observados, como la birrefringencia. 


\section{Bibliografía}

[1] P. Ball, The Self-Made Tapestry: Pattern Formation in Nature, Oxford University Press, 1999.

[2] D'Arcy W. Thompson, On Growth and Form, Cambridge University Press, 1992.

[3] G. Nicolis, I. Prigogine, Self-organization in non-equilibrium systems: : from dissipative structures to order through fluctuations, Wiley \& Sons, 1977.

[4] D. Kondepudi, I. Prigogine, Modern Thermodynamics, John Wiley \& Sons, 1998.

[5] L. Wolpert, The triumph of Embryo, Dover Pub. Inc, 2008.

[6] I.R. Epstein, J.A. Pojman, An introduction to non-linear chemical dynamics, Oxford University Press, 1998.

[7] L.G. Harrison, Kinetic theory of living pattern, Cambridge University Press, 1993.

[8] R.J. Field, M. Burguer (eds.), Oscillations and travelling waves in chemical systems, Wiley-Interscience, 1985.

[9] S. Kondo, T. Miura, Reaction-Diffusion Model as a Framework for Understanding Biological Pattern Formation, Science, 329, p. 1616, 2010. 
[10] P.K. Maini, K.J. Paintera, H.N. Phong Chaub, Spatial pattern formation in chemical and biological systems, J. Chem. Soc. Faraday Trans., 93(20), p. 3601, 1997.

[11] A. M. Turing, The Chemical Basis of Morphogenesis, Philosophical Transactions of the Royal Society of London. Series B, Biological Sciences, 641, p. 37, 1952.

[12] L.A. Segel (ed.), Mathematical models in molecular and cellular biology, Cambridge University Press, 1980.

[13] W.Y. Ning et al, Turing Patterns in a Reaction-Diffusion System, Commun. Theor. Phys, 45, p. 761, (2006).

[14] V. Castets, E. Dulos, 3. Boissonade, P. De Kepper, Experimental Evidence of a Sustained Standing Turing-Type Nonequilibrium Chemical Pattern, Phys. Rev. Lett., 64, p. 2953, 1990.

[15] P. De Kepper, V. Castets, E. Dulos and J. Boissonade, Turing-type chemical patterns in the chlorite-iodide-malonic acid reaction, Physica D, 49, p. 161, 1991.

[16] Q. Ouyang, H.L. Swinney, Transition from a uniform state to hexagonal and striped Turing patterns, Nature (London), 352, p. 610, 1991.

[17] Q. Ouyang, H.L. Swinney, Pattern formation in the presence of symmetries, Phys. Rev. E, 50, p. 2802, 1994.

[18] M. Watzl, A.F. Munster, Turing-like spatial patterns in a polyacrylamide-methylene blue-sulfide-oxygen system, Chem. Phys. Lett., 242, p. 273, 1995.

[19] K. Lee, W. D. McCormick, Q. Ouyang, H. L. Swinney, Pattern Formation by Interacting Chemical Fronts, Science, 261, p. 192, 1993.

[20] K. Lee, W. D. McCormick, J. E. Pearson, H. L. Swinney, Experimental observation of self replicating spots in a reaction-diffusion system, Nature (London), 369, p. 215, 1994.

[21] I. Lengyel, S. Kádár, I.R. Epstein, Transient Turing Structures in a Gradient-Free Closed System, Science,259, p. 493, 1993. 
[22] V.K. Vanag, I.R. Epstein, Chapter 5: Patterns of Nanodroplets: The Belousov-Zhabotinsky-Aerosol OT-Microemulsion System, of Selforganized morphology in nanostructured materials, Springer, Berlin, 2008 .

[23] H. Liu,J.A. Pojman, Y. Zhao, C. Pan, J. Zheng, L.Yuan, A.K. Horvát, Q. Gao, Pattern formation in the iodate-sulfite-thiosulfate reactiondiffusion system, Phys. Chem. Chem. Phys., 2012, Advance Article.

[24] J.D. Murray, Lecture notes on nonlinear differential equation models in biology, Clarendon Press, Oxford, 1979.

[25] J.A. Sánchez Colmenarejo, Formación de patrones biológicos: sistemas de Reacción-Difusión, Revista Antena de Telecomunicación, junio, 2009.

[26] S.H. Strogratz, Nolinear dynamics and chaos, Perseus Books, 1994.

[27] R. Seydel, Bifurcation and stability analysis. From equilibrium to chaos, Second edition, "Interdisciplinary Applied Mathematics", volume 5, Springer, 1994.

[28] P. Gray, Sthepen Scott, Chemical oscillations and inestabilities, Clarendon Press, Oxford, 1990.

[29] I. Prigogine, R. Lefever, Dissipative structures in chemical systems, J. Chem. Phys., 48, 1695-1700, 1968.

[30] L.H. Juárez Valencia, Notas del curso Modelos y Problemas de Difusión, 2010.

[31] J.D. Murray, Mathematical Biology: I, An introduction, Third edition, Ïnterdisciplinary Applied Mathematics", volume 17, Springer, 2003.

[32] J.D. Murray, Mathematical Biology: II. Spatial Models and Biomedical Applications, Third edition, Ïnterdisciplinary Applied Mathematics", Volume 18, Springer, 2003.

[33] L.H. Juárez Valencia, Notas del curso Diferencias finitas e Ecuaciones Diferenciales Parciales, 2006. 
[34] S.J. Ruuth, Implicit-explicit methods for reaction-diffusion problems in pattern formation, Journal of Mathematical Biology, Vol. 42, 2, p. 148, 1995.

[35] L.H. Juárez Valencia, Notas del curso Introducción al Elemento Finito, 2006.

[36] E. Becker, G. Carey, J.T. Oden, Finite Elements, an introducion, Vol 1., Prentice Hall, 1981.

[37] http://www.gidhome.com/

[38] A. Madzvamuse, Time stepping schemes for moving grid finite elements applied to reaction diffusion systems on fixed and growing domains, Journal of Computational physics, 214, p. 239, 2006.

[39] V. Dufiet, J. Boissonade, Numerical studies of Turing patterns selection in a two-dimensional system, Physica A, 188, p. 158, 1992.

[40] J.E. Pearson, Complex patterns in a simple system, Science,261, pp. 189-192, 1993.

[41] R. A. Barrio, C. Varea, J. L. Aragón, P. K. Maini, A Two-dimensional Numerical Study of Spatial Pattern Formation in Interacting Turing Systems, Bulletin of Mathematical Biology, 61, p. 483, 1999.

[42] C.H. Galeano et al, Formación de patrones de turing para sistemas de reacción-convección-difusión en dominios fijos sometidos a campos de velocidad toroidal, Rev. Fac. Ing. Univ. Antioquia, 53, p. 75, 2010.

[43] J. Schnakenberg, Simple chemical reaction systems with limit cycle behaviour, Journal of Theoretical Biology, 81, p. 389, 1979.

[44] U.A. Buitrago-Suárez, Anatomía comparada y evolución de las especies Pseudoplatystoma Bleeker 1862 (Siluformes: Pimelodidae), Rev. Acad. Colom. Cien., 114, p. 113, 2006.

[45] U.A. Buitrago-Suárez , B. M. BURR, Taxonomy of the catfish genus Pseudoplatystoma Bleeker (Siluriformes: Pimelodidae) with recognition of eight species, Zootaxa, 1522, p. 1, 2007. 
[46] P.P. Padilla-Pérez, F. Alcántara-Bocanegra, R. Orbe, Reproducción inducida de la doncella Pseudoplatystoma fasciatum y desarrollo embrionario-larval, Folia Amazonica, 12, p. 141, 2001.

[47] K.J. Painter, Models for pigment pattern formation in the skin of fishes, Mathematical Models for Biological Pattern Formation, 121, p. 59, 2001

[48] D.M. Parichy, Pigment patterns: fish in stripes and spots, Current Biology, 24, p. R947, 2003.

[49] S. Kondo, R. Asai, A reaction-diffusion wave on the skin of the marine angelfish pomacanthus. Nature, 376, p. 675, 1995.

[50] C. Varea, J. L. Aragon, R. A. Barrio, Confined turing patterns in growing systems, Phys. Rev. E., 56, p. 1250, 1997.

[51] H. Meinhardt, The algorithmic beauty of sea shells, Springer, Berlin, New York, 2nd edition, 1998.

[52] K. J. Painter, P. K. Maini, H. G. Othmer, Stripe formation in juvenile Pomacanthus explained by a generalized Turing mechanism with chemotaxis, PNAS, 10, p. 5549, 1999.

[53] R. A. Barrio, R.E. Baker, B. Vaughan, K. Tribuzy, M.R. de Carvalho, R. Bassanezi, P.K. Maini, Modeling the skin pattern of fishes, Phys. Rev E, 79, p. 031908, 2009

[54] C. Varea, D. Hernández, R. A. Barrio, Soliton behaviour in a bistable reaction diffusion model, J. Math. Biol., 54, p. 797, 2007.

[55] P. Arcuri, J. D. Murray, Pattern sensitivity to boundary and initial conditions in reaction-diffusion models, J. Math. Biol., 24, p. 141, 1986.

[56] A.R. Sanderson, R.M. Kirby, C.R. Johnson, L.Yang, Advanced Reaction-Diffusion Models for Texture Synthesis, J. Graphics GPU Game Tools, 11, p.47, 2006.

[57] H. Shoji, Y.Iwasa, A. Mochizuki, S. Kondo, Directionality of Stripes Formed by Anisotropic Reaction Diffusion Models, J. theor. Biol., 214, p. 549, 2002. 
[58] A. Madzvamuse, P.K. Maini, A.J. Wathen, A Moving Grid Finite Element Method for the Simulation of Pattern Generation by Turing Models on Growing Domains, Journal of Scientific Computing, 2, p. 194, 2005 .

[59] S. Suthersan, Remediation engineering: design concepts, CRC Press, 1996.

[60] S. Schuster, M. Marhl, T Hofer, Modelling of simple and complex calcium oscillations: From single-cell responses to intercellular signalling, Eur. J. Biochem., 269, p. 1333, 2002.

[61] H. Híjar, D. Marquina de Hoyos, I. Santamaría-Holek, Pattern formation from consistent dynamical closures of uniaxial nematic liquid crystals, J. Chem. Phys., 136, p. 114109, 2012.

[62] A. Duobova, F. Guillén González, Un curso de análisis numérico: interpolación, aproximación, integración y resolución de ecuaciones diferenciales, Universidad de Sevilla, 2007.

[63] J.C. Strikwerd, Finite Difference Schemes and Partial Differential Equations, 2nd Edition, SIAM, 1989.

[64] P. Solín, Partial Differential Equations and the Finite Element Method, John Wiley and Sons, 2006.

[65] A. Ern, J.L. Guermond, Theory and Practice of Finite Elements, Volume 159 of Applied Mathematical Sciences, Springer, 2003. 


\section{Apéndice A. Error, estabilidad y convergencia en métodos de diferencias finitas}

A continuación ilustraremos algunas de las propiedades más importantes de los esquemas numéricos de diferencias finitas ${ }^{18}$. Esto lo hacemos únicamente para la ecuación de difusión, omitiendo los términos no lineales de la reacción, pues esto introduce una complejidad en los cálculos ajenos al propósito de este trabajo. Así pues, con fines meramente ilustrativos consideremos la ecuación de difusión en una dimensión:

$$
\frac{\partial w}{\partial t}=D \frac{\partial^{2} w}{\partial y^{2}}
$$

definida en un dominio $\Omega=[0,1]$ y con condiciones tipo Dirchlet en la frontera $\Gamma: w(y \in \Gamma)=0$. Sea $U_{j}^{n}$ la aproximación de $u$ al tiempo $n \Delta t$ y en la posición $y=j h$, siendo $h$ el tamaño del elemento de la malla y $\Delta t$ el paso de tiempo.

\section{Error de truncamiento y orden del esquema}

Para fijar ideas, consideremos el método explícito que aproxima la ecuación anterior (1) por el siguiente esquema de diferencias:

\footnotetext{
${ }^{18}$ La información de esta sección así como los detalles de los distintos cálculos pueden verse en $[35,62,63]$.
} 


$$
\frac{W_{j}^{n+1}-W_{j}^{n}}{\Delta t}=\frac{D}{h^{2}}\left(W_{j+1}^{n}-2 W_{j}^{n}+W_{j}^{n}\right) .
$$

El error de truncamiento $T(y, t)$ para un de diferencias finitas se obtiene cuando la aproximación $W_{j}^{n}$ se reemplaza por la solución exacta de la ecuación diferencial. Es decir:

$$
T(y, t)=\frac{w(y, t+\Delta t)-w(y, t)}{\Delta t}-\frac{D}{h^{2}}(w(y+h, t)-2 w(y, t)+w(y-h, t)) .
$$

Sustituyendo $w(y, t), w(y+\Delta t)$ y $w(y-\Delta t)$ por sus respectivas expansiones de Taylor y omitiendo términos de orden más alto a $\Delta t$ y a $h^{2}$, se obtiene que el error de truncamiento es:

$$
T(y, t)=\frac{1}{2} w_{t t}(y, \eta) \Delta t-\frac{1}{12} w_{y y y y}(\xi, t) h^{2},
$$

con $\xi \in(y-h, y+h), \eta \in(t, t+\Delta t)$, donde el subíndice de $w$ significan derivada parcial respecto a la variable. De esta igualdad puede verse que $T(y, t) \rightarrow 0$ cuando $\Delta t, h \rightarrow 0$. Esto significa que el esquema de diferencias (2) es consistente con la ecuación diferencial (1), puesto que éste se acerca a la ecuación diferencial cuando los parámetros de discretización tienden a cero.

Por otro lado, si las derivadas de $\left|w_{t t}\right|$ y $\left|w_{y y y y}\right|$ están acotadas por $M_{t t} \mathrm{y}$ $M_{\text {yyyy }}$ respectivamente en el dominio, entonces :

$$
|T(y, t)| \leq \frac{\Delta t}{2}\left(M_{t t}+\frac{M_{y y y y}}{6 \mu}\right),
$$

donde $\mu=(D \Delta t) / h^{2}$. Esto significa que al fijar el valor de $\mu,|T(y, t)|$ tiende a cero asintóticamente tan rápido como $\Delta t$. Por lo tanto, se dice que el esquema es de primer orden en $\Delta t$. Otro tanto igual se puede demostrar para un esquema implícito de primer orden, mientras que el esquema 2-SBDF es de segundo orden. En el caso bidimensional, el método de direcciones alternantes es de segundo orden.

\section{Análisis de Fourier del error y estabilidad}

La solución analítica de la ecuación (1) se puede obtener como la suma infinita de los modos de Fourier: 


$$
w(y, t)=\sum_{-\infty}^{\infty} a_{m} w_{m}(y, t)=\sum_{-\infty}^{\infty} a_{m}\left[e^{-k^{2} t} e^{i k y}\right],
$$

donde los $a_{k}$ son los coeficientes de Fourier que dependen de las condiciones iniciales. A $k=m \pi$ (con $m$ entero) se le llama el número de onda, pues esta relacionado con el número de oscilaciones que tienen cada modo de Fourier $w_{m}(y, t)$ dentro del dominio. Es fácil ver que los modos de Fourier más alto tienden más rápidamente a cero (pues su amplitud decae como $e^{-m \pi t}$ ), y que son los más oscilatorios (con $m / 2$ ondas en el el dominio).

En el caso de la solución aproximada $W_{j}^{n}=W(j h, n \Delta t)$, se puede definir un modo de Fourier discreto de la forma:

$$
W_{i}^{n}(k)=\sigma^{n} e^{i k(j h)}
$$

A $\sigma(k)$ se le conoce como factor de amplificación ${ }^{19}$. Al sustituir (3) en la ecuación en diferencias (2), uno obtiene que este factor está dado por:

$$
\sigma(k)=1-4 \mu \sin ^{2}(h k / 2) .
$$

Es posible mostrar que la diferencia entre los modos de Fourier en el caso continuo y discreto difieren poco para bajas frecuencia, i.e. para modos de Fourier bajos. Sin embargo, se puede mostrar que si $\mu>1 / 2,|\sigma(k)|>1$ por lo que la amplitud de las oscilaciones de los modos más altos de la aproximación discreta crecen desmesuradamente ${ }^{20}$. Como hemos visto, esto no sucede en el caso continuo, por lo que se estaría introduciendo error. Esto significa que el método explícito es condicionalmente estable. La condición

$$
\mu=\frac{D \Delta t}{h^{2}} \leq \frac{1}{2}
$$

significa que, dada una malla fija con elementos de tamaño $h \ll 1$, el intervalo de tiempo debe ser menor a $h^{2} /(2 D)$. Es decir, conforme más precisión

${ }^{19}$ Esta definición se hace en analogía al modo de Fourier continuo:

$$
w\left(y_{j}, t_{n}\right)=w(j h, n \Delta t)=e^{-k n \Delta t} e^{i k(j h)}=\left(e^{-k \Delta t}\right)^{n} e^{i k(j h)},
$$

por lo que ahora $\sigma$ sustituye al término entre paréntesis.

${ }^{20}$ Aquí conviene señalar que, a diferencia de la solución analítica, los modos de Fourier discretos no son infinitos. Si recordamos que la oscilación del modo m-ésimo de Fourier requiere de al menos $m+1$ nodos, el número más alto de Fourier discreto es M-1, siendo $\mathrm{M}$ el número de nodos totales. 
espacial se necesite, menor debe ser el paso de tiempo, y por ende, mayor el número de iteraciones necesarias para cubrir el tiempo total. Esta limitación no ocurre en los esquemas implícitos que son incondicionalmente estables, ni en el método de direcciones alternantes.

\section{Convergencia y la ecuación RD}

Si se cumple la condición anterior, hemos visto que el esquema explícito es consistente y estable. Según un teorema atribuido a P. Lax, esto garantiza que el método sea convergente. Esto significa que si $\mu \leq 1 / 2$ la solución aproximada $W_{j}^{n}$ tiende al valor exacto de la solución cuando los parámetros de discretización $\Delta t$ y $h$ tienden a cero. En el caso de los métodos implícitos que son incondicionalmente estable, junto con la consistencia, el teorema demuestra la convergencia para toda $\mu$.

Como hemos dicho antes, en los cálculos de esta sección no hemos considerado los términos de reacción, los cuales no son lineales. El análisis del error en este caso no es trivial debido a que aparece un problema que no surge en el caso de ecuaciones lineales: el error se propaga de manera no-lineal cuando $n$ crece. Sin embargo, tanto Ruuth [34], como Madzvamuse [38] han evaluado el error de distintos esquemas numéricos de diferencias finitas aplicados específicamente a las ecuaciones RD. Sus resultados confirman que los esquemas de diferencias finitas son adecuados si se escoge un intervalo de tiempo suficientemente pequeño. Ver Figura 11. 

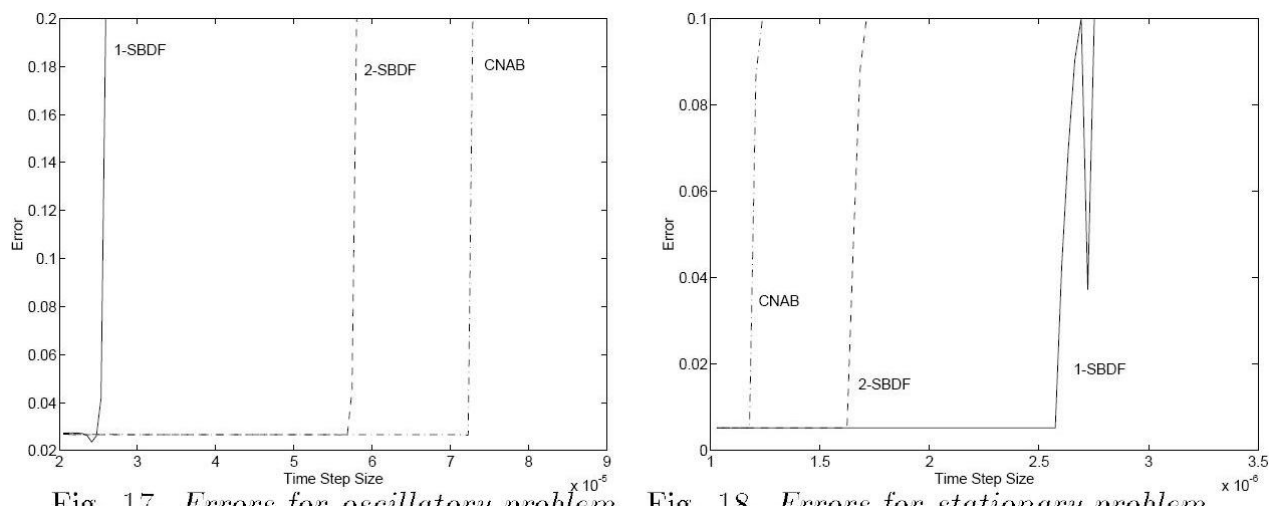

Fig. 17. Errors for oscillatory problem

Fig. 18. Errors for stationary problem

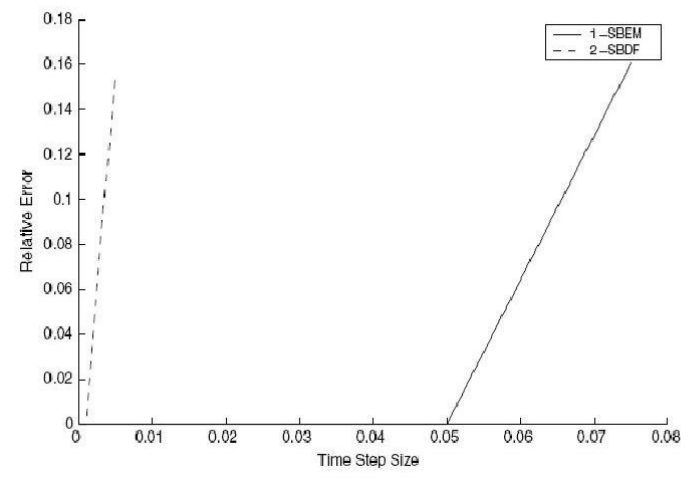

Figura 11: Cuantificación del error numérico de distintos esquemas de diferencias finitas para ecuaciones RD (reacción de Schnakenberg), como función del intervalo de tiempo. Izquierda: Imagen de Ruuth [34]; el error para los métodos 1-SBDF, 2-SBDF Y CNAB que es un Crank-Nicholson para los términos de difusión y tipo Adams-Bashfort para los términos de reacción. En esta imagen se considera una solución puramente oscilatoria. Derecha: Del mismo autor, se comparan estos métodos para una solución con patrones estacionarios. Abajo: De Madzvamuse [38]; se comparan el esquema 2-SBDF con un esquema de Euler hacia atrás 1-SBEM. 


\section{Apéndice B. Resolución de la ecuación reacción convección}

La ecuación que estudiamos es de la forma:

$$
\frac{\partial u}{\partial t}=R(u)+d \nabla^{2} u+\mathbf{z} \cdot \nabla u
$$

con una ecuación similar para $v$. Para simplificar la exposición y sin pérdida de generalidad, resolveremos esta ecuación para el caso unidimensional y sólo para cuando $\mathbf{z}=-z \hat{e}_{y}$. En este caso y en analogía con el método 2-SBDF, las distintas derivadas de $u$ se aproximarán mediante las siguientes diferencias finitas:

$$
\begin{gathered}
\frac{\partial u}{\partial t} \approx \frac{3 U_{j}^{n+1}+4 U_{j}^{n}-U_{j}^{n-1}}{2 \Delta t} \\
\nabla^{2} u=\frac{\partial^{2} u}{\partial y^{2}} \approx \frac{U_{j+1}^{n}-2 U_{j}^{n}+U_{j-1}^{n}}{h^{2}}, \\
\mathbf{z} \cdot \nabla u=-z \frac{\partial u}{\partial y} \approx-z\left(\frac{U_{j+1}^{n+1}-U_{j-1}^{n+1}}{2 h}\right)
\end{gathered}
$$

Al sustituir esto en la ecuación (4), mediante un poco de álgebra se obtiene:

$(\nu-\mu) U_{j+1}^{n+1}+(3-2 \mu) U_{j}^{n+1}-(\nu+\mu) U_{j-1}^{n+1}=2 \Delta t\left(R\left(U_{j}^{n}\right)-R\left(U_{j}^{n-1}\right)\right)+4 U_{j}^{n}-U_{j}^{n-1}$, 
con $\mu=2 d \Delta t / h^{2}$ y $\nu=z \Delta t / h$. La ecuación anterior representa un sistema de ecuaciones para $U^{n+1}$ donde el vector de la derecha depende de la solución en los tiempos anteriores $n$ y $n-1$. Nótese que la matriz ya no es simétrica lo cual indica en este caso el movimiento preferencial en una dirección. 


\section{Apéndice C. Teorema de Lax-Milgram}

En este apartado mencionaremos el teorema que permite garantizar la convergencia del método de Elemento Finito ${ }^{21}$. Para ello, consideremos el problema elíptico (4.9) en una dimensión, con coeficiente $\nu=1$ y con fronteras tipo Neumann en $x=0$ y $x=1$ :

$$
\begin{gathered}
-u^{\prime \prime}+u=f \quad \text { en } \Omega \\
\frac{\partial u}{\partial n}=0 \quad \text { en } \delta \Omega
\end{gathered}
$$

La forma variacional de este problema puede escribirse en analogía con (4.12) como:

$$
a(u, v)=L(v)
$$

donde se han usado los operadores:

$$
\begin{gathered}
a(u, v)=\int_{\Omega}\left(u v+u^{\prime} v^{\prime}\right) d \Omega \\
L(v)=\int_{\Omega} f v d \Omega
\end{gathered}
$$

con ambas funciones definidas en el espacio:

$$
V=\left\{v: \int_{\Omega}|v|^{2}+\left|v^{\prime}\right|^{2} d \Omega<\infty\right\} .
$$

\footnotetext{
${ }^{21}$ La información de este apartado se obtuvo de [64, 65].
} 


\section{Observaciones}

1) El espacio $V$ tienen un producto escalar y una norma definidos por:

$$
\begin{gathered}
(v, w)_{V}:=\int_{\Omega}\left(v w+v^{\prime} w^{\prime}\right) d \Omega \\
\|v\|_{V}:=\left(\int_{\Omega}\left(v^{2}+\left|v^{\prime}\right|^{2}\right) d \Omega\right)^{1 / 2} .
\end{gathered}
$$

Dado que $V$ es completo, entonces es un espacio de Hilbert y es conocido como el espacio de Sobolev de orden 1.

2) La forma $a(v, w)$ es claramente bilineal y simétrica. Además es Velíptica coercitiva. Esto significa que existe $k_{1}>0$ tal que $a(v, v) \leq k_{1}\|v\|_{V}^{2}$, lo cual es evidente de la definición:

$$
a(v, v)=\int_{\Omega}\left(v^{2}+\left|v^{\prime}\right|^{2}\right) d \Omega=\|v\|_{V}^{2},
$$

por lo que basta tomar $k_{1}=1$. Por otro lado se puede mostrar que la forma $a(v, w)$ es continua, es decir, que existe $k_{2}>0$ tal que $|a(v, w)| \leq$ $k_{2}\|v\|_{V}\|w\|_{V}$. Esto se puede demostrar notando que la forma $a(v, w)$ coincide con el producto interior de $V$ y luego usando la desigualdad de CauchySchwarz:

$$
\begin{gathered}
a(v, w)=\int_{\Omega}\left(v w+v^{\prime} w^{\prime}\right) d \Omega=(v, w)_{V}, \text { por lo que } \\
|a(v, w)|=\left|(v, w)_{V}\right| \leq\|v\|_{V}\|w\|_{V}
\end{gathered}
$$

por lo que basta tomar $k_{2}=1$.

3) La forma $L(v)$ es continua, es decir, existe $k_{3}$ constante finita tal que $|L(v)| \leq\|v\|_{V}$. Para ver esto, nótese que:

$$
|L(v)|^{2}=\left(\int_{\Omega} f v d \Omega\right)^{2} \leq \int_{\Omega}(f v)^{2} d \Omega \leq\left(\int_{\Omega} f^{2} d \Omega\right)\left(\int_{\Omega} v^{2} d \Omega\right)
$$


por lo que

$$
|L(v)|^{2} \leq\left(\int_{\Omega} f^{2} d \Omega\right)\left(\int_{\Omega}\left(v^{2}+\left|v^{\prime}\right|^{2}\right) d \Omega\right)=\|f\|_{L_{2}}\|v\|_{V} .
$$

Esto significa que basta tomar $k_{3}=\|f\|_{L_{2}}$ finita para demostrar que $L(v)$ es continua.

Estas propiedades hacen que se cumplan las hipótesis del teorema de Lax-Milgram:

Teorema 1 Si la forma lineal $L(v)$ es continua y la forma bilineal $a(u, v)$ es $V$-elíptica y continua en el espacio de Hilbert $V$, entonces el problema de encontrar $v \in V$ tal que:

$$
a(u, v)=L(v)
$$

tiene solución única $u \in V$ y satisface la cota de estabilidad:

$$
\|u\|_{V} \leq \frac{k_{3}}{k_{1}}
$$

Así pues, este teorema garantiza que la solución del problema variacional (4.12) corresponde con la solución exacta de la ecuación diferencial original (4.9). Ahora estudiaremos las hipótesis que hacen posible que la solución aproximada obtenida por el método de elemento finito converja a la solución del problema variacional y, por ende, a la solución exacta.

\section{El método de Galerkin}

Este método proporciona una forma sistemática de obtener aproximaciones de dimensión finita al problema variacional. Para ello se considera una familia de funciones $\left\{V_{h}\right\}_{h>0}$ de subespacios de dimensión finita de $V$. Supongamos que $\forall v \in V$ existe una sucesión $v_{h}$ tal que :

$$
v_{h} \rightarrow v \quad \text { cuando } \quad h \rightarrow 0,
$$

es decir, $h$ representa un parámetro de refinamiento que, cuanto más pequeño sea, la función de dimensión finita se aproxima más a la función original de dimensión infinita en principio. Si $L$ pertenece al espacio dual de $\mathrm{V}$, en la aproximación de Galerkin se trata de encontrar $u_{h} \in V_{h}$ tal que: 


$$
a\left(u_{h}, v_{h}\right)=L\left(v_{h}\right) \quad \forall v_{h} \in V_{h} .
$$

Si definimos una base de $V_{h}$ dada por $\left\{\phi_{j}: j=1, \ldots, N\right\}$ donde $N$ es la dimensión del subespacio, entonces la solución aproximada se puede escribir como una combinación lineal de estas funciones según:

$$
u_{h}=\sum_{j=1}^{N} \xi_{j} \phi_{j} .
$$

Como hemos visto, esto resulta en un sistema algebraico de ecuaciones dado por:

$$
\mathbb{A} \vec{\xi}=\vec{L}
$$

con $\mathbb{A}$ la matriz de componentes $a\left(\phi_{i}, \phi_{j}\right), \vec{L}$ el vector de componentes $L\left(\phi_{j}\right)$ y $\vec{\xi}$ el vector incógnita con las coordenadas de la solución aproximada $u_{h}$ en la base finita. En nuestro problema (4.14), la matriz $\mathbb{A}$ es la suma de la matriz de masa y de rigidez. El siguiente teorema garantiza la convergencia de la solución aproximada a la variacional cuando el parámetro de discretización $h$ tiende a cero.

Teorema 2 Bajo las hipótesis del teorema de Lax-Milgram, para todo $h>0$ existe una única solución $u_{h} \in V_{h}$ del problema discreto:

$$
a\left(u_{h}, v_{h}\right)=L\left(v_{h}\right) \quad \forall v_{h} \in V_{h},
$$

Ésta satisface la cota de estabilidad: $\left\|u_{h}\right\|_{V} \leq \frac{\|L\|_{V^{\prime}}}{k_{1}}$. Además, se tiene que:

$$
\left\|u-u_{h}\right\|_{V} \leq \frac{k_{3}}{k_{1}} \inf _{v_{h} \in V_{h}}\left\|u-u_{h}\right\|_{V}
$$

donde $u$ y $u_{h}$ son las soluciones del problema variacional y discreto respectivamente. En particular, si $u_{h} \rightarrow u$ cuando $h \rightarrow 0$, entonces el método de Galerkin converge. 


\section{Apéndice D. Listado de los programas utilizados}

A continuación presentamos algunas muestras representativas de los programas utilizados en esta tesis.

\begin{tabular}{|c|c|}
\hline Página & Método \\
\hline \hline I & Explícito (EXP) \\
\hline II & Semi-implícito de primer orden (1-SBDF) \\
\hline V & Semi-implícito de segundo orden (2-SBDF) \\
\hline VII & Semi-implícito de direcciones alternantes (ADI) \\
\hline \hline X & Elemento finito \\
\hline
\end{tabular}




\section{PROGRAMA 1. DIFERENCIAS FINITAS. MÉTODO EXPLÍCITO}

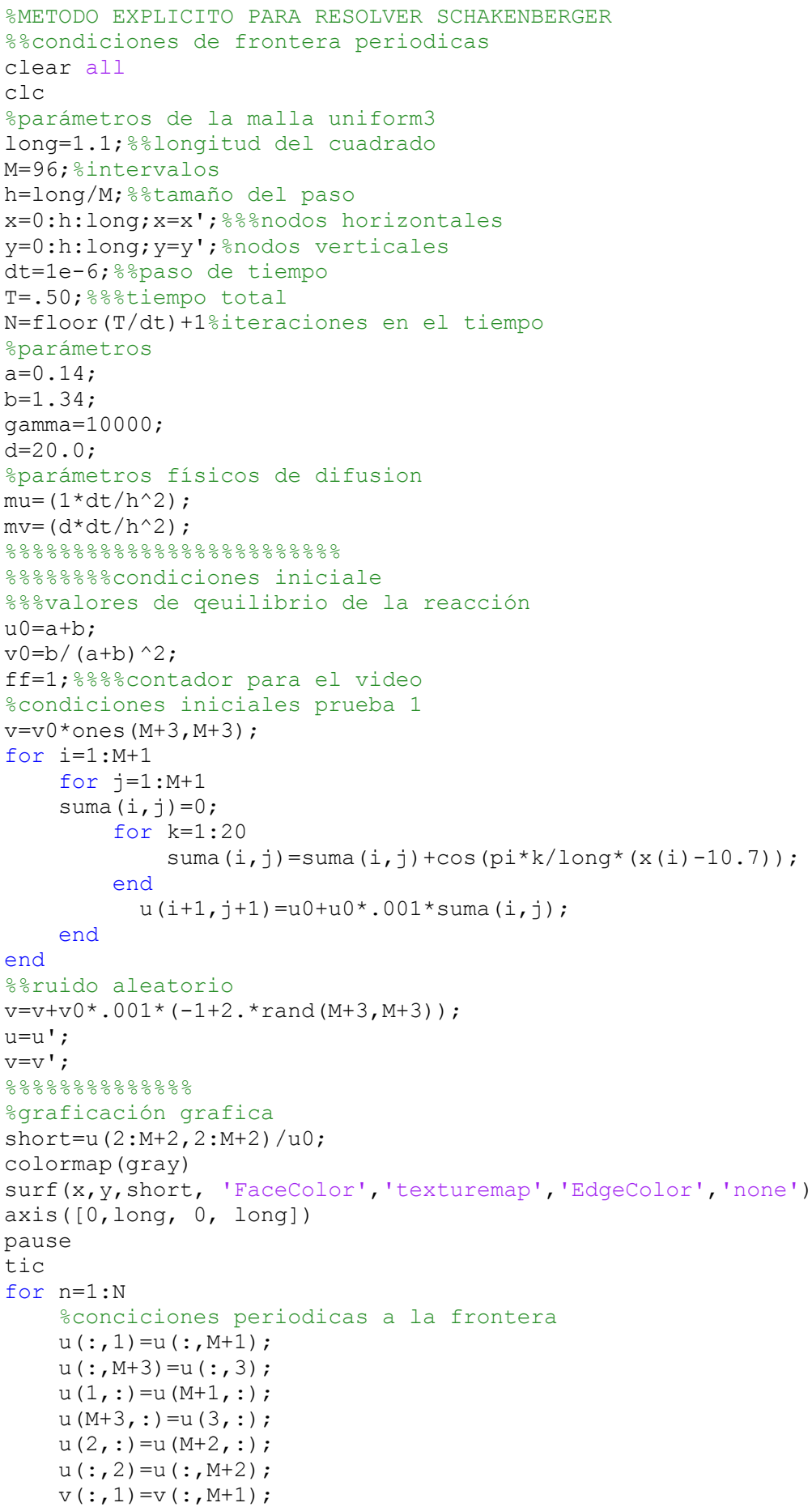




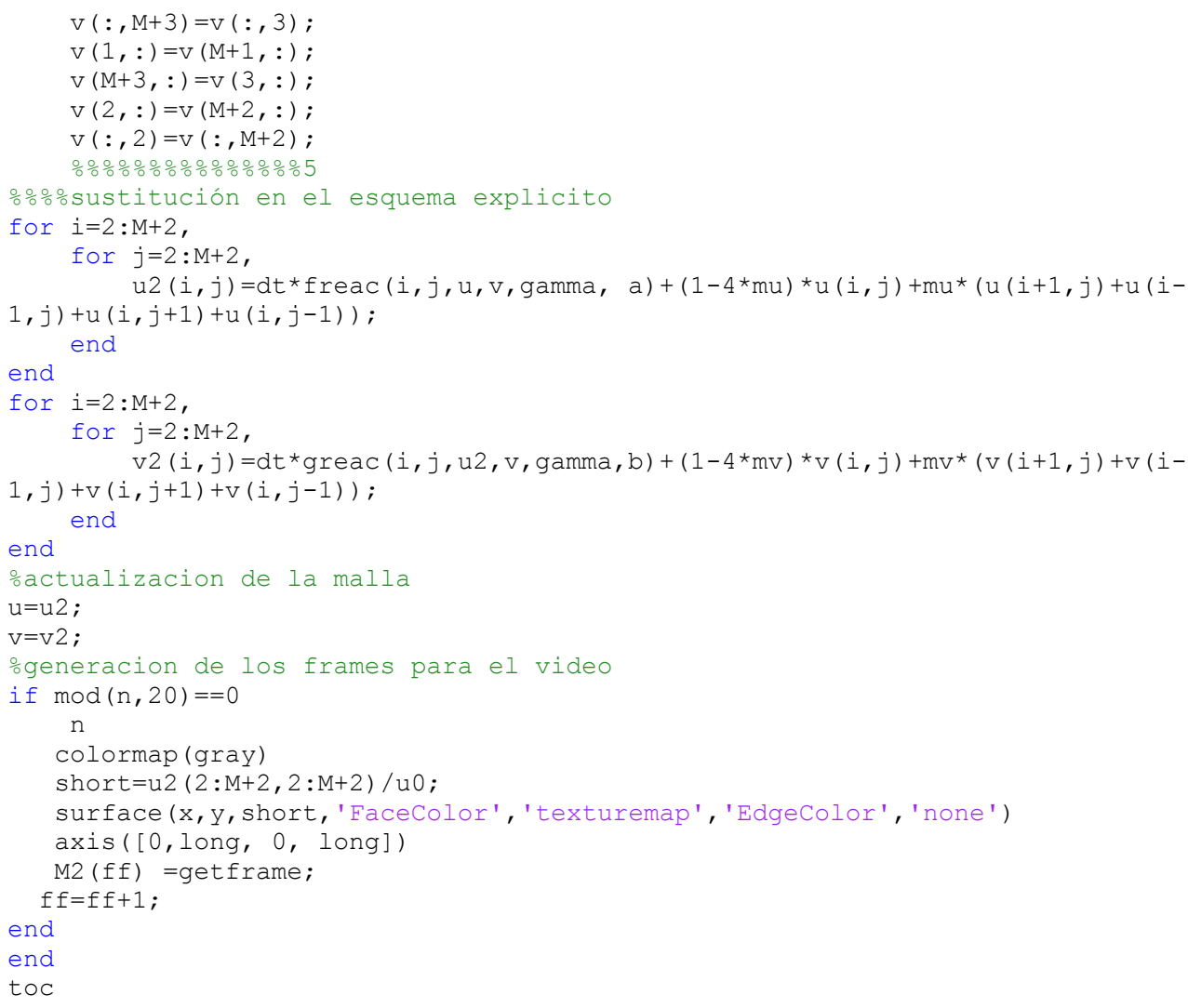

\section{PROGRAMA 2.- DIFERENCIAS FINITAS, MÉTODO SEMI-IMPLÍCITO PRIMER ORDEN}

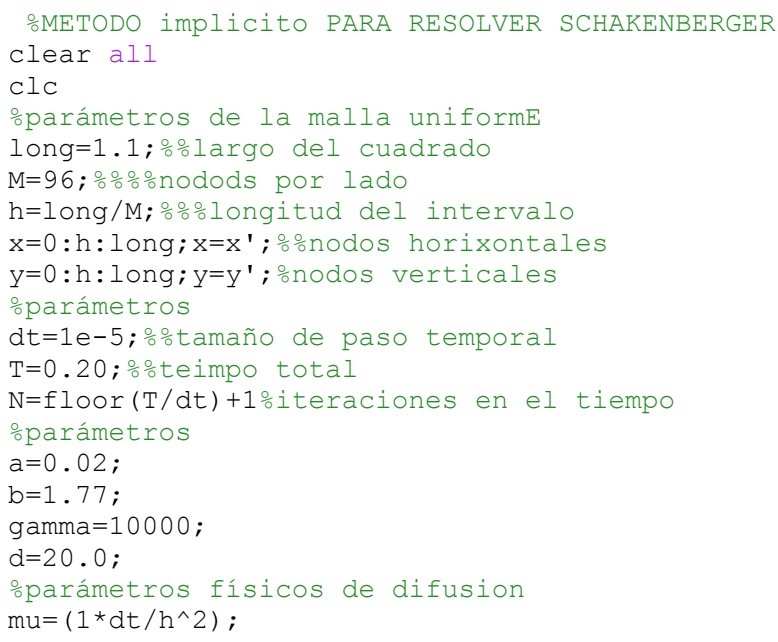




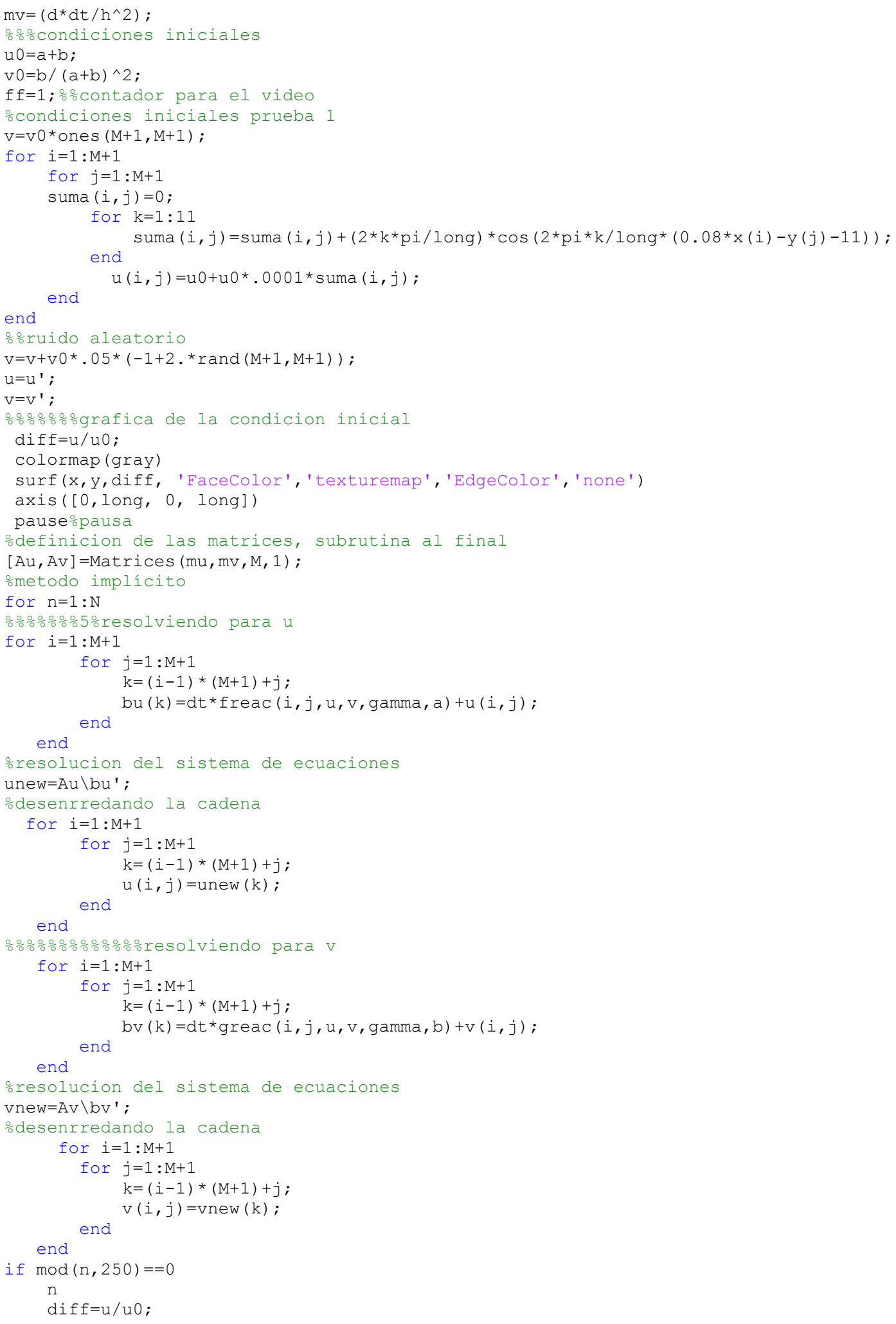




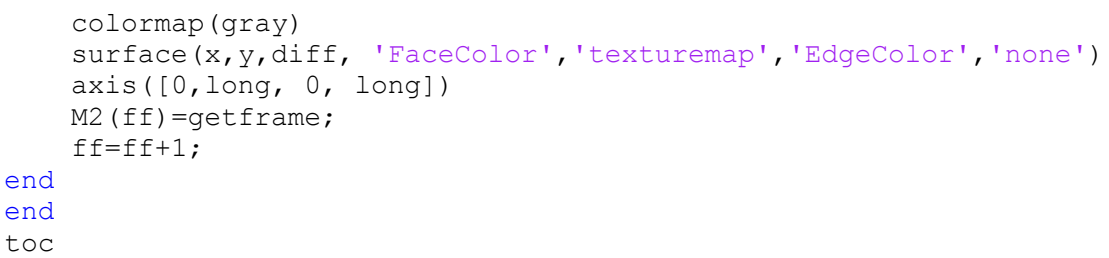




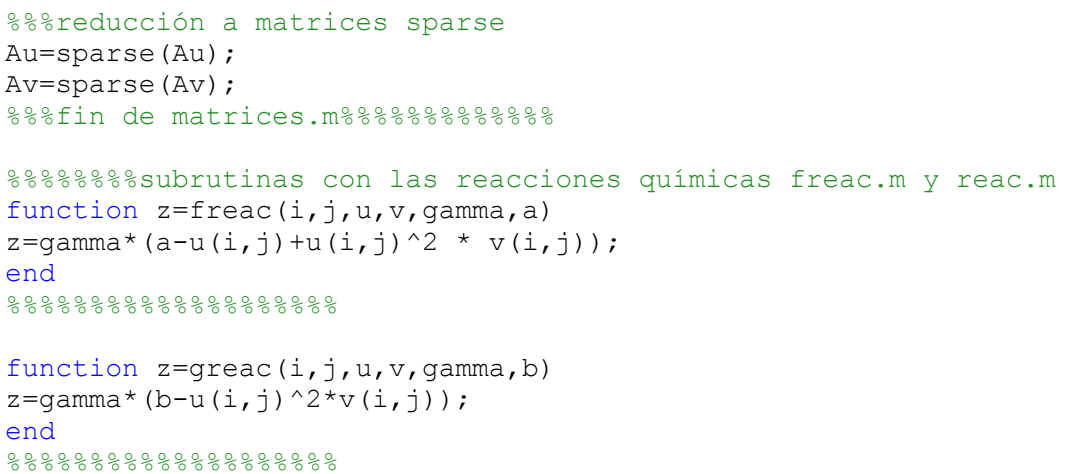

\section{PROGRAMA 3.- DIFERENCIAS FINITAS, MÉTODO SEMI-IMPLICITO DE SEGUNDO ORDEN}

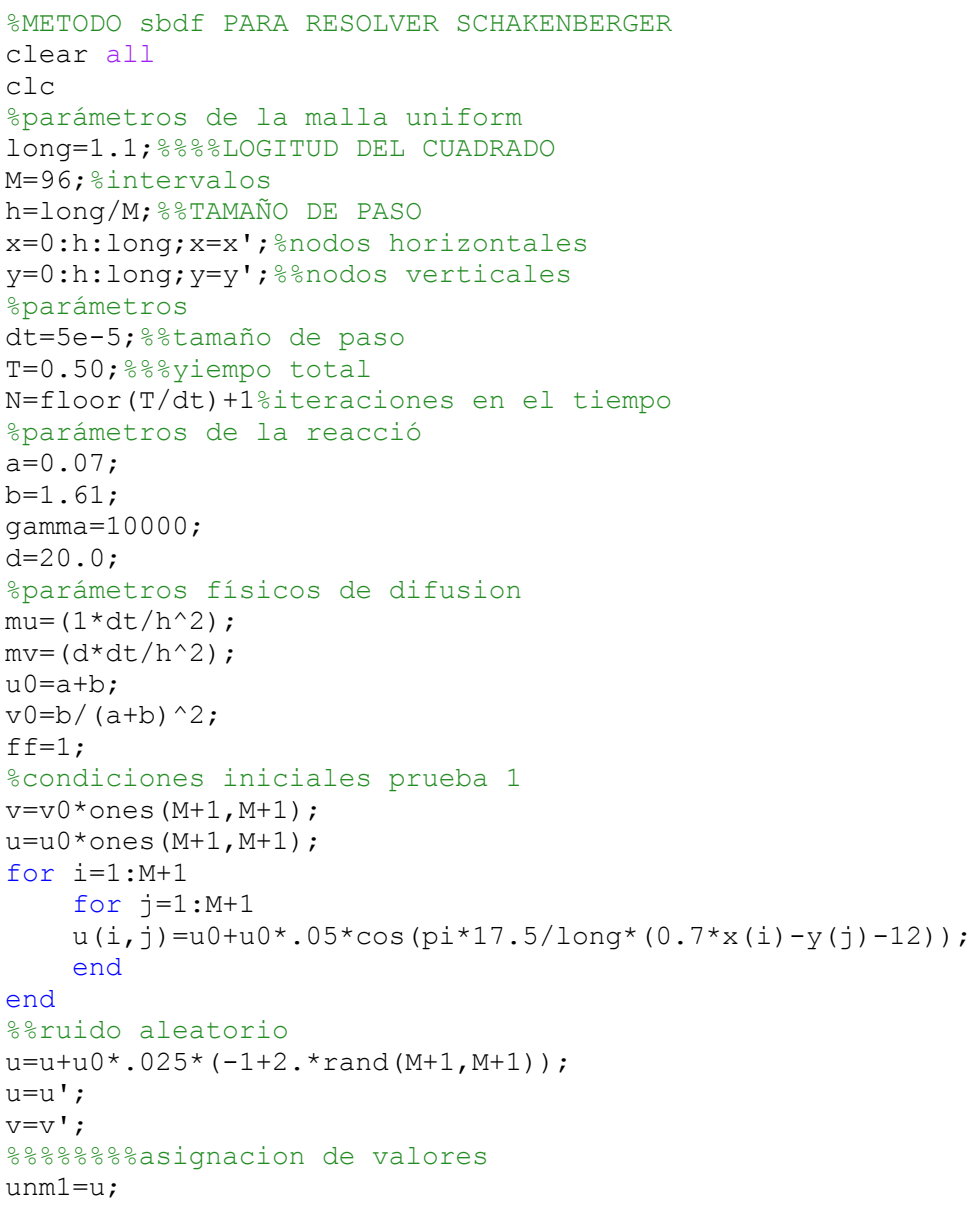




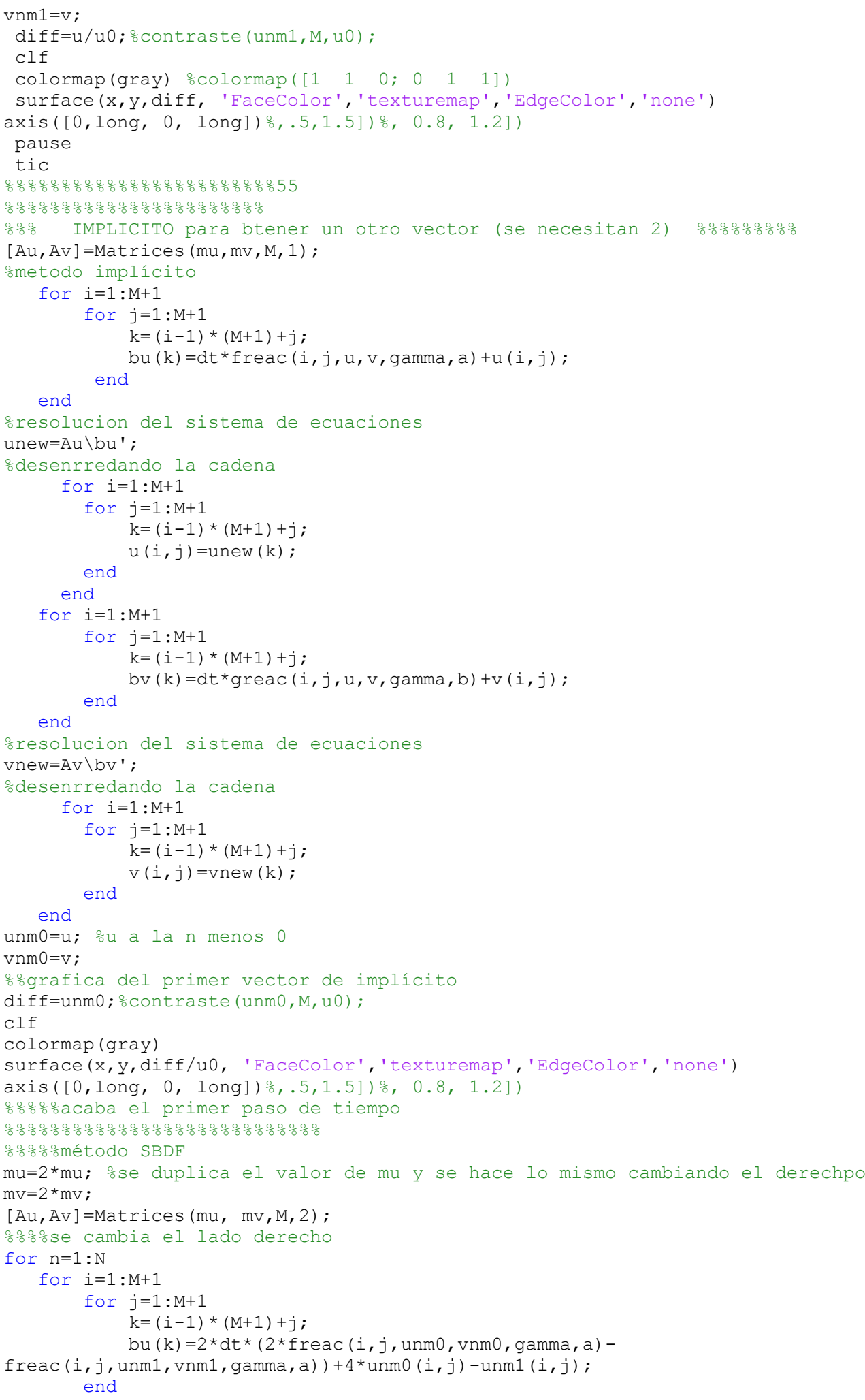




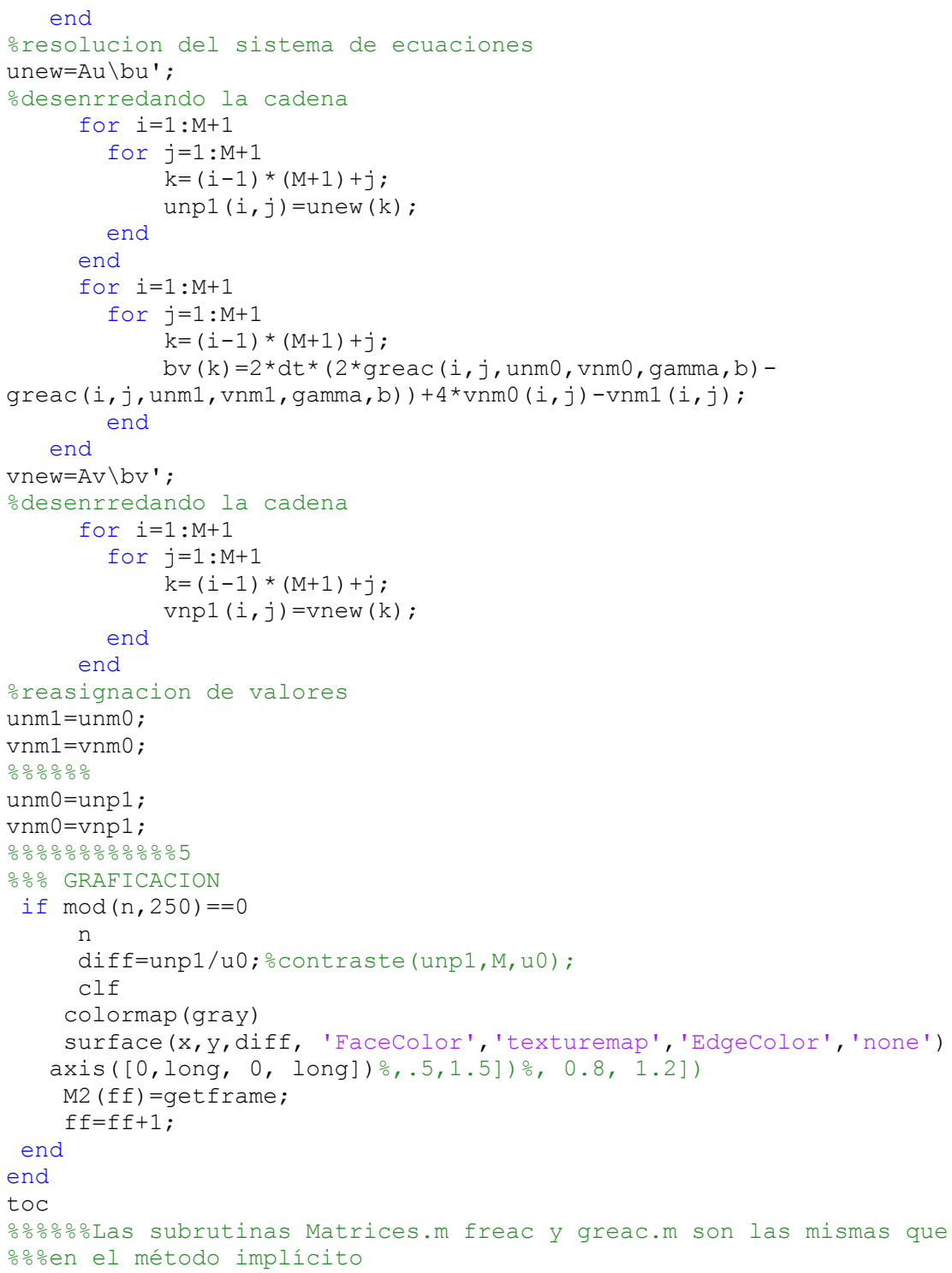

\section{PROGRAMA 4. DIFERENCIAS FINITAS CON EL MÉTODO ADI}

-METODO adi para schnakenberg

clear all

clc

oparámetros de la malla uniform

long=2; \%otamaño del cuadrado 


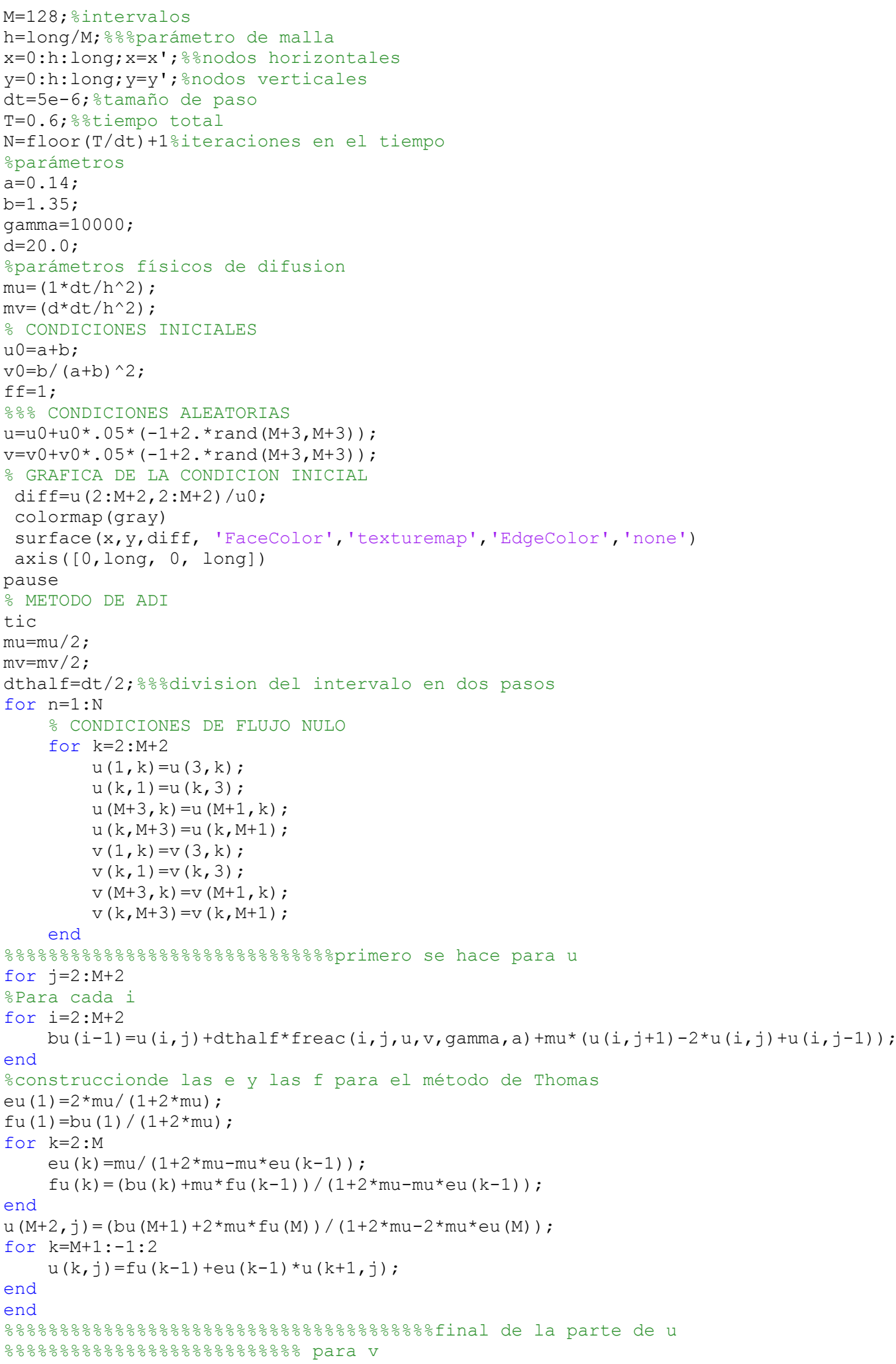




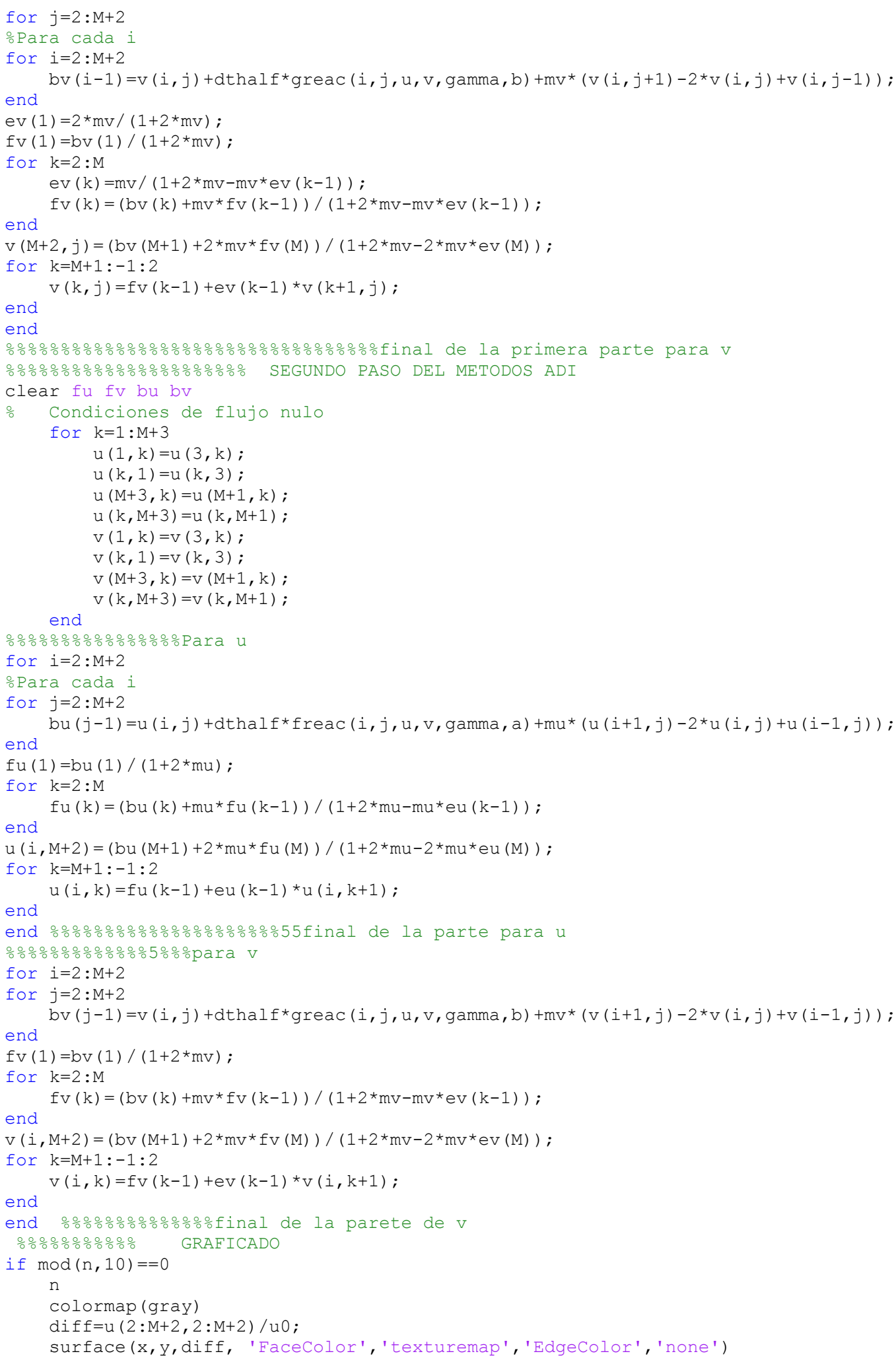




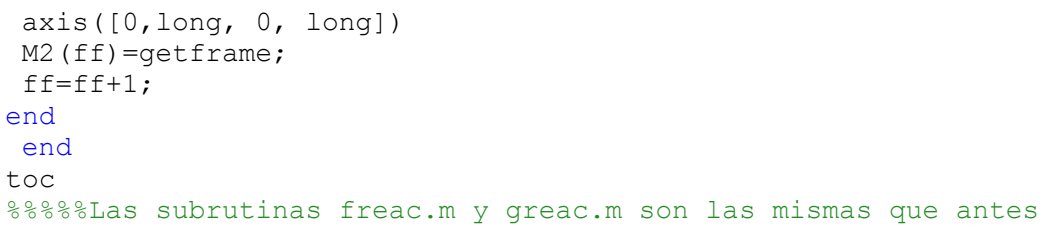

\section{PROGRAMA 5. ELEMENTO FINITO (SOLO LA RUTINA PRINCIPAL)}

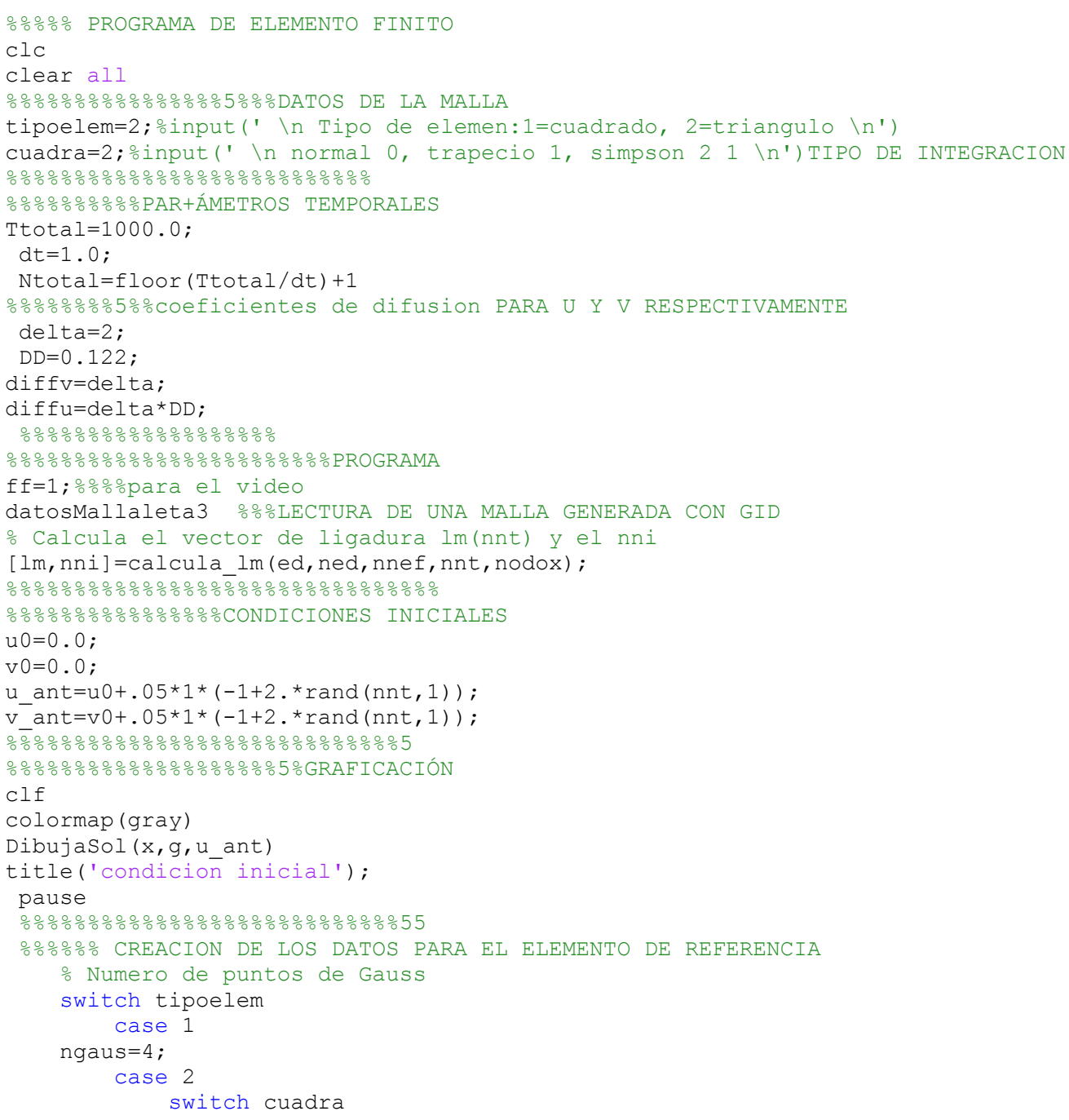




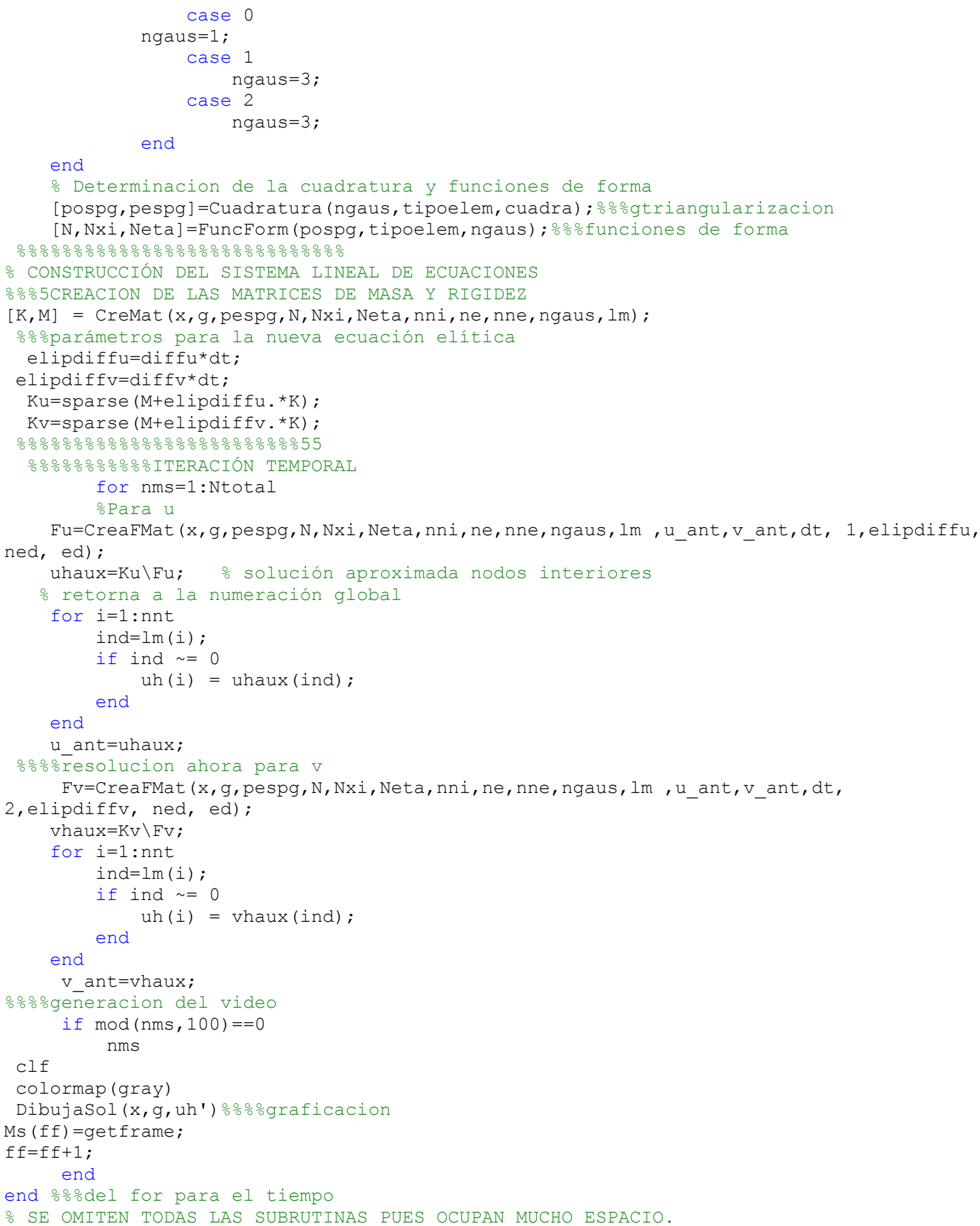

